\title{
ON THE GROWTH RATE OF TURBULENT MIXING LAYERS: A NEW PARAMETRIC MODEL
}

\author{
A Thesis \\ presented to \\ the Faculty of California Polytechnic State University, \\ San Luis Obispo
}

\begin{abstract}
In Partial Fulfillment
of the Requirements for the Degree

Master of Science in Aerospace Engineering
\end{abstract}

by

Jeffrey Lee Freeman

February 2014 
(C) 2014

Jeffrey Lee Freeman

ALL RIGHTS RESERVED 


\section{COMMITTEE MEMBERSHIP}

TITLE: $\quad$ On the Growth Rate of Turbulent Mixing Layers: A New Parametric Model

AUTHOR: $\quad$ Jeffrey Lee Freeman

DATE SUBMITTED: $\quad$ February 2014

COMMITTEE CHAIR: Dr. Dianne DeTurris, Professor Aerospace Engineering Department California Polytechnic State University, San Luis Obispo

COMMITTEE MEMBER: Dr. Jin Tso, Professor Aerospace Engineering Department California Polytechnic State University, San Luis Obispo

COMMITTEE MEMBER: Dr. David Marshall, Professor Aerospace Engineering Department California Polytechnic State University, San Luis Obispo

COMMITTEE MEMBER: Brett G. Morham, MS, Industry Advisor Northrop Grumman, Aerospace Systems Branch Redondo Beach, CA 


\section{ABSTRACT \\ On the Growth Rate of Turbulent Mixing Layers: A New Parametric Model Jeffrey Lee Freeman}

A new parametric model for the growth rate of turbulent mixing layers is proposed. A database of experimental and numerical mixing layer studies was extracted from the literature to support this effort. The domain of the model was limited to planar, spatial, nonreacting, free shear layers that were not affected by artificial mixing enhancement techniques. The model is split into two parts which were each tuned to optimally fit the database; equations for an incompressible growth rate were derived from the error function velocity profile, and a function for a compressibility factor was generalized from existing theory on the convective Mach number. The compressible model is supported by a detailed evaluation of the currently accepted models and practices, including error analysis of the convective Mach number derivation and a critical analysis of Slessor's renormalization technique which affected his 1998 compressibility parameter. Analysis of the database suggested that a distinction should be made between thickness definitions that are based on the velocity profile and those based on the density profile. Additionally, the accumulation of different normalization approaches throughout the literature was shown to have introduced non-physical variance in the trends. Resolution of this issue through a consistent normalization process has greatly improved the normality and scatter of the data and the goodness-of-fit of the models, resulting in $R^{2}=0.9856$ for the incompressible model and $\mathrm{R}^{2}=0.9004$ for the compressible model.

Keywords: Mixing Layer, Shear Layer, Turbulent, Compressible, Growth Rate 


\section{ACKNOWLEDGMENTS}

My utmost gratitude goes out to my advisor, Dr. Dianne DeTurris, whose enthusiasm and perspective have been both motivating and educational. Our weekly meetings will be sorely missed.

I also wish to thank my parents for their unending support and encouragement, for I certainly wouldn't be where I am today without them, and Suzie for her understanding and patience during those long days and nights at the library, coffee shops, computer labs, and so on. To the people in my life who have tolerated me and my preoccupation during these years, I thank you all.

Finally, I must pay respect to the countless researchers whose contributions to the study of aerodynamics have enabled my own. Today, I stand on your shoulders. 


\section{Table of Contents}

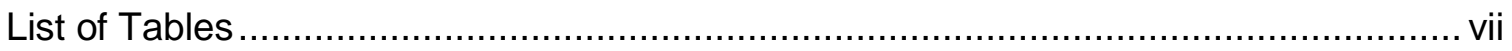

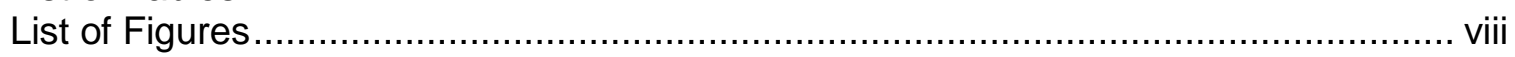

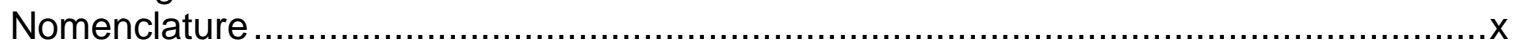

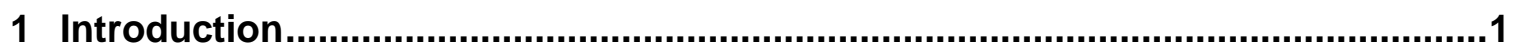

1.1 Basic Characteristics of Turbulent Mixing Layers..........................................

1.1.1 Mixing Layer Thickness Definitions................................................

1.2 Incompressible Mixing Layer Velocity Profile Model......................................6

1.3 Compressibility Effects on the Turbulent Mixing Layer Growth Rate ................10

1.3.1 Observed Characteristics of Compressibility..............................10

1.3.2 Measures of Compressibility...............................................11

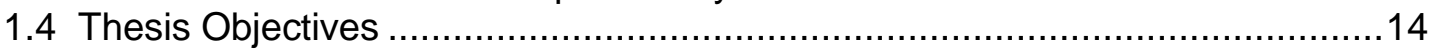

2 The Database .................................................................................................16

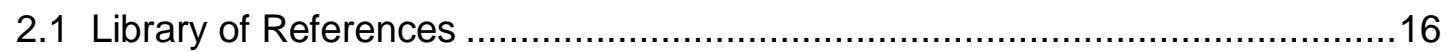

2.2 Data Qualification Rubric .................................................................. 17

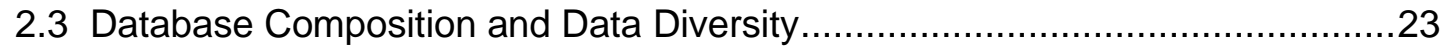

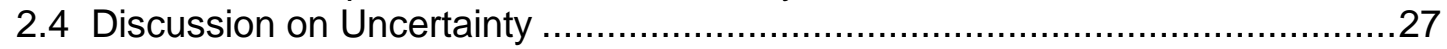

2.4.1 Reported Uncertainty within the Articles ...................................27

2.4.2 Errors Introduced in the Collation of the Database ..........................28

2.5 Unaltered Representation of the Database ................................................. 30

3 Deeper Analysis of Existing Models ................................................................32

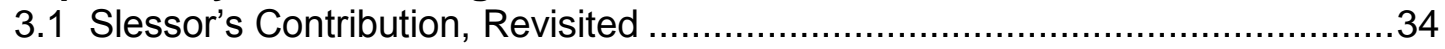

3.1.1 Evaluation of Slessor's Re-Normalization Technique.....................34

3.1.2 Evaluation of Slessor's Compressibility Parameter ..........................38

3.2 The Convective Mach Number, Revisited ................................................. 40

3.3 Normalization and the Incompressible Growth Rate Model............................46

3.3.1 Derivation of the Incompressible Growth Rate Model .....................46

3.3.2 Apparent Dependence on Mixing Layer Thickness Definition ..........49

3.3.3 Inconsistent Normalization Case Example: Data from Chinzei et al. (1986).

3.4 Dimotakis (1991a) Trendline, Revisited ...................................................54

4 A New Model for Turbulent Mixing Layer Growth Rates ....................................58

4.1 Discussion of Statistical Tools Used During the Modeling Process ..................58

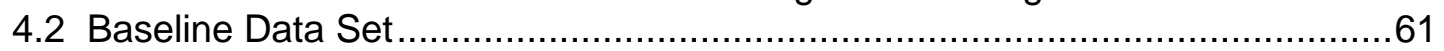

4.3 Tuning the Incompressible Growth Rate Model ........................................63

4.3.1 Application of Coefficient Functions for Incompressible Growth Rate Model Tuning ...........................................................63

4.3.2 Updated Data Set with Consistently Applied Incompressible

Growth Rate Model............................................................69

4.4 Identification and Removal of Outliers ............................................... 71

4.5 Final Data Set and Fitted Model .............................................................. 74

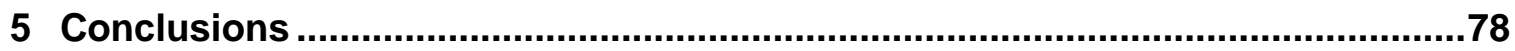

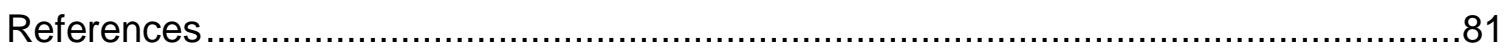

Appendix A: Complete Library Reference List ................................................ 84

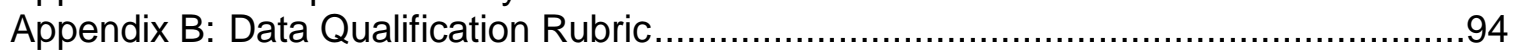

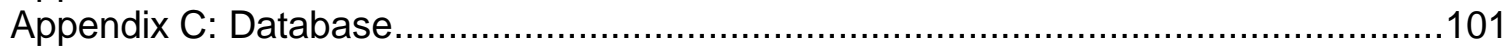

Appendix D: Pertinent Article Summaries and Comments on Data Extraction .............111 


\section{List of Tables}

Table 1. Incompressible mixing layer data set.

Table 2. Comparison of incompressible model coefficients for $\sigma_{0}=11.0$ and $\sigma_{0}=$ 10.3773 


\section{List of Figures}

Figure 1. An iconic shadowgraph image of a turbulent mixing layer. From Brown and Roshko (1974)....

Figure 2. Graphical representation of Pitot thickness definition for wake-like defects. From Papamoschou and Roshko (1988).

Figure 3. Comparison of the error function velocity profile and the hyperbolic tangent velocity profile, and their effect on velocity-based mixing layer thicknesses. In both cases, $\sigma_{0}=11.0$.

Figure 4. Error function velocity profile plotted for a fixed $\lambda_{s}$ over many $x$-locations.........9

Figure 5. Self-similarity demonstrated in the error function velocity profile....................10

Figure 6. Normalized mixing layer thickness growth rates plotted against convective Mach number. From Smits and Dussauge (2006).

Figure 7. The application of Slessor's compressibility parameter to a collection of experimental growth rate data. Solid curve is a best-fit line. From Slessor (1998)......14

Figure 8. Histogram of velocity ratios within the database. .......................................25

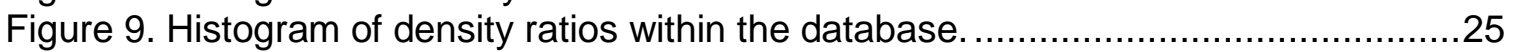

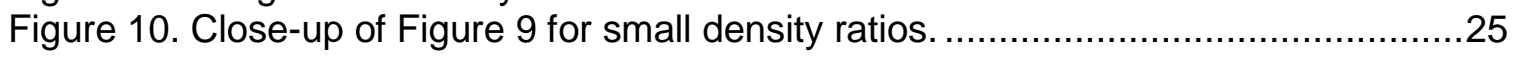

Figure 11. Histogram of mixing parameters within the database .................................26

Figure 12. Histogram of convective Mach numbers within the database.......................26

Figure 13. Histogram of acoustic speed ratios within the database..............................26

Figure 14. The unaltered mixing layer database represented in terms of convective

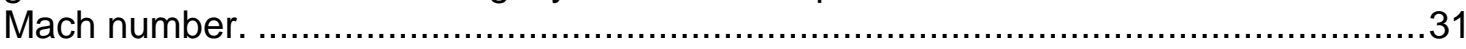

Figure 15. The unaltered mixing layer database represented in terms of Slessor's compressibility parameter................................................................................

Figure 16. Slessor's initial data set, reproduced from Figure 6.1a of his 1998 thesis. ....35

Figure 17. Slessor's re-normalized data set, reproduced from Figure 6.1b of his 1998 thesis.

Figure 18. Slessor's re-normalized data set plotted against his compressibility parameter, reproduced from Figure 6.4 of his 1998 thesis.

Figure 19. Sketches of streamlines of a turbulent mixing layer for (a) stationary frame of reference, and (b) convective frame of reference. From Papamoschou and Roshko (1988).

Figure 20. Percent error introduced to $M_{c_{2}}=f\left(M_{c_{1}}\right)$ relationship by the omission of $O\left(M_{C_{1}}^{4}\right)$ terms.

Figure 21. The unaltered mixing layer database represented in terms of convective Mach number and identified by thickness definition type. .....................................

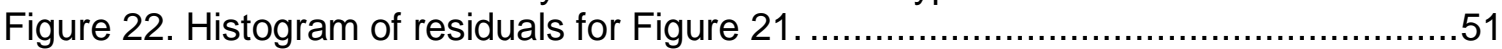

Figure 23. Various representations of the growth rates observed by Chinzei et al. (1986).

Figure 24. The effect of model parameter "A".

Figure 25. The effect of model parameter "B".

Figure 26. The effect of model parameter "C"

Figure 27. A sample boxplot, from Hodge and Austin (2004) ....................................60

Figure 28. Data Set \#0 - Unmodified velocity-based data set with regression................62

Figure 29. Data Set \#0 - Histogram of residuals. ......................................................63

Figure 30. Boxplot of residuals for the first pass of incompressible growth rate model tuning. 
Figure 31. Boxplot of residuals for the second pass of incompressible growth rate model tuning

Figure 32. Boxplot of residuals for the third pass of incompressible growth rate model tuning.

Figure 33. Histogram of residuals for the incompressible growth rate model.

Figure 34. Data Set \#1 - Consistently normalized by incompressible growth rate model, $\sigma_{0}=10.3773$.

Figure 35. Data Set \#1 - Histogram of residuals.

Figure 36. Boxplot of residuals for the first pass of outlier identification for Data Set \#1....

Figure 37. Boxplot of residuals for the second pass of outlier identification for Data Set \#2.

Figure 38. Six outliers shown alongside Data Set \#2

Figure 39. Data Set \#2 - Final data set - Outliers removed................................... 74

Figure 40. Data Set \#2 - Histogram of residuals.

Figure 41. Percent difference between Data Set \#2 and its associated model. .76 


\section{Nomenclature}

\begin{tabular}{|c|c|c|c|}
\hline$A, B, C$ & Model parameters & $c$ & Convective \\
\hline$C_{\delta}$ & Growth rate coefficient & def & Thickness definition $(b, S, \omega$, \\
\hline$M$ & Mach number & & pit, etc.) \\
\hline$P$ & Pressure & eff & Effective \\
\hline$R$ & Specific gas constant, $\mathrm{J} / \mathrm{kg} / \mathrm{K}$ & obs & Observed \\
\hline$R^{2}$ & Coefficient of determination & pit & Pitot thickness \\
\hline$T$ & Temperature & $s$ & Static \\
\hline$U$ & Velocity & $t$ & Total \\
\hline$U^{*}$ & Normalized velocity & vis & Visual thickness \\
\hline$a$ & Acoustic speed & $\theta$ & Momentum thickness \\
\hline$h$ & Enthalpy & $\rho m$ & Maximum concentration \\
\hline$r$ & Velocity ratio, $U_{2} / U_{1}$ & & thickness \\
\hline$s$ & Density ratio, $\rho_{2} / \rho_{1}$ & $\omega$ & Vorticity thickness \\
\hline$t$ & Time & & \\
\hline$x$ & Streamwise direction & & \\
\hline$y$ & Transverse direction & & \\
\hline$\Pi_{c}$ & $\begin{array}{l}\text { Slessor's compressibility } \\
\text { parameter }\end{array}$ & & \\
\hline$\alpha$ & $\begin{array}{l}\text { Error from non-ideal gasses } \\
\text { and different static pressures }\end{array}$ & & \\
\hline$\gamma$ & Ratio of specific heats & & \\
\hline$\delta$ & Mixing layer thickness & & \\
\hline$\delta^{\prime}$ & Growth rate of $\delta, \partial \delta / \partial x$ & & \\
\hline$\delta_{0}^{\prime}$ & $\begin{array}{l}\text { Incompressible growth rate for } \\
\text { equivalent } r \text { and } s\end{array}$ & & \\
\hline$\varepsilon$ & Error from $M_{c}$ simplification & & \\
\hline$\theta$ & $\begin{array}{l}\text { Momentum boundary layer } \\
\text { thickness }\end{array}$ & & \\
\hline$\lambda$ & Mixing parameter & & \\
\hline$\rho$ & Density & & \\
\hline$\rho^{*}$ & Concentration & & \\
\hline$\sigma_{0}$ & Spreading parameter & & \\
\hline$\phi$ & Normalized growth rate, $\delta^{\prime} / \delta_{0}^{\prime}$ & & \\
\hline$\hat{\phi}$ & Normalized growth rate model & & \\
\hline
\end{tabular}

\section{Subscripts}

1 Primary stream

2 Secondary stream

$S \quad$ Stanford thickness

$b \quad$ Shear layer thickness 


\section{Introduction}

The turbulent mixing layer has been a subject of research for over seventy years. Since it is a core phenomenon within the processes of entrainment and fuel injection, scientists and engineers alike have been steadily seeking a thorough understanding of its behavior and accurate predictive capabilities. This report presents a collation of the existing literature, reviews of some prominent theories, and the introduction of a new predictive model with improved accuracy.

\subsection{Basic Characteristics of Turbulent Mixing Layers}

Turbulent mixing layers are formed when two dissimilar streams of turbulent fluid travel parallel to each other. Commonly, the streams are initially separated by a thin, low angle splitter plate. The high-speed flow is designated as the primary stream with subscript "1", while the low-speed flow is designated as the secondary stream with subscript "2". Conventionally, mixing layers have been identified by their velocity ratio,

$$
r=\frac{U_{2}}{U_{1}}
$$

and their density ratio,

$$
s=\frac{\rho_{2}}{\rho_{1}}
$$

Figure 1 shows a shadowgraph image of a mixing layer created by Brown and Roshko (1974). The images taken during that study uncovered a very characteristic trait of turbulent mixing layers to be the large, coherent turbulent structures that travel downstream between the two fluids. These structures are caused by a Kelvin-Helmholtz instability at the junction of the two streams. They have been observed to grow as they 
travel downstream, and therefore are often used to identify the extent of the mixing layer thickness, $\delta$.

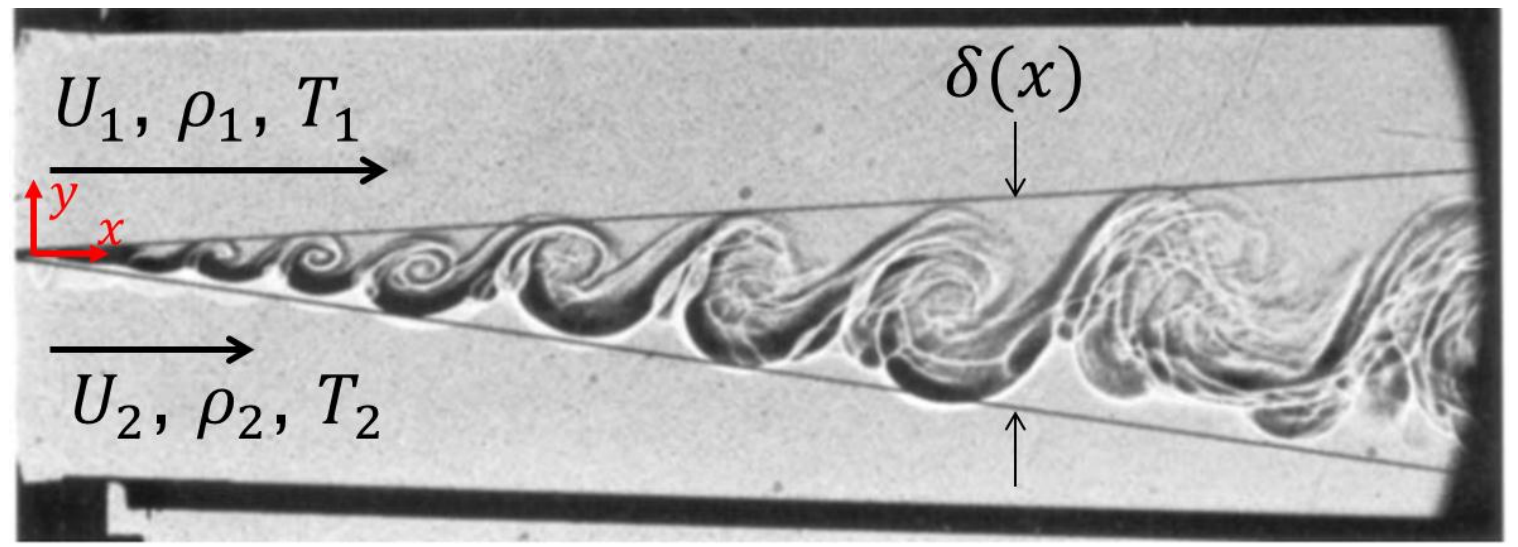

Figure 1. An iconic shadowgraph image of a turbulent mixing layer. From Brown and Roshko (1974).

Turbulent mixing layers have also proven to be self-similar when sufficiently downstream of the splitter plate. For low values of $x(x=0$ at the end of the splitter plate), the mixing layer thickness has a nonlinear growth rate and the mixing layer behaves more like a wake flow. Further downstream, the turbulent mixing becomes the dominant factor and the mixing layer is self-similar. In these regions, the transverse ( $y$-direction) velocity profiles from any $x$-value should stack on top of one another when normalized by the mixing layer thickness. Furthermore, the mixing layer thickness growth rate, $\delta^{\prime}$, is constant in the selfsimilar region.

\subsubsection{Mixing Layer Thickness Definitions}

Throughout the years, a variety of different mixing layer thickness definitions have arisen. This is due largely to the types of data that each individual researcher has available from his/her experiment. Thicknesses are commonly defined by some combination of the velocity profile and the density profile. Additionally, thickness estimates can be extracted 
from images of the flow, which can be taken using a variety of imaging techniques including (but not limited to) shadowgraph and Schlieren. Some researchers have suggested that direct relationships can be drawn between some of the different thickness definitions.

\section{The Shear Layer Thickness, $\boldsymbol{\delta}_{b}$}

The shear layer thickness, $\delta_{b}$, is the distance between transverse $(y)$ locations where $U^{*}=$ 0.1 and $U^{*}=0.9$. The normalized velocity, $U^{*}$, is defined as

$$
U^{*}(y)=\frac{U(y)-U_{2}}{U_{1}-U_{2}}
$$

The values of 0.1 and 0.9 were chosen to account for experimental uncertainty. At the theoretical limits of $U^{*}=0$ and $U^{*}=1$, the freestream turbulent intensity accounts for a larger portion of measured deviations from the nominal freestream velocity. The $10 \%$ buffer on either side is appropriately sized to ensure that the edge of the mixing layer is identified with reasonable certainty. This thickness has also been referred to as the velocity $10 \%$ thickness because of the conventionally accepted size of this buffer.

\section{The Stanford Thickness, $\delta_{S}$}

The Stanford thickness, $\delta_{S}$, is the distance between transverse locations where $U^{*}=\sqrt{0.1}$ and $U^{*}=\sqrt{0.9}$. This thickness has also been referred to as the energy thickness because it resembles the shear layer thickness for $\left(U^{*}\right)^{2}$ rather than $U^{*}$. This thickness definition gained its popularity at the 1980-81 AFOSR-HTTM-Stanford Conference on Complex Turbulent Flows (Kline et al. 1983).

\section{The Vorticity Thickness}

The vorticity thickness, $\delta_{\omega}$, is defined as 


$$
\delta_{\omega}=\frac{\Delta U}{[\partial U / \partial y]_{\max }}
$$

where

$$
\Delta U=U_{1}-U_{2}
$$

Eq. 5

\section{The Momentum Thickness, $\boldsymbol{\delta}_{\boldsymbol{\theta}}$}

The momentum thickness, $\delta_{\theta}$, is defined as

$$
\delta_{\theta}=\int_{-\infty}^{+\infty} \frac{\rho}{\rho_{1}} U^{*}\left(1-U^{*}\right) d y,
$$

Eq. 6

\section{The Maximum Concentration Thickness, $\boldsymbol{\delta}_{\rho m}$}

The maximum concentration thickness, $\delta_{\rho m}$, is calculated by joining the $20 \%$ and $80 \%$ points of the concentration profiles with a straight line and measuring the distance between the intercepts of this line and the $0 \%$ and $100 \%$ concentration levels. Concentration is defined as

$$
\rho^{*}(y)=\frac{\rho(y)-\rho_{1}}{\rho_{2}-\rho_{1}}
$$

\section{The Pitot Thickness, $\delta_{\text {pit }}$}

The Pitot thickness, $\delta_{p i t}$, has the same form as the shear layer thickness, except that it is based on a normalized total pressure instead of a normalized velocity and the buffer is reduced to $5 \%$. That is, it is defined as the distance between transverse locations where $P_{t}^{*}=0.05$ and $P_{t}^{*}=0.95$. The normalized total pressure, $P_{t}^{*}$, is defined as

$$
P_{t}^{*}(y)=\frac{P_{t}(y)-P_{t, 2}}{P_{t, 1}-P_{t, 2}} .
$$

If the profile has a wake-like defect, it is split into a top and bottom part at the location of the minimum; $\delta_{p i t}$ is then measured from $95 \%$ of the bottom difference with respect to the 
minimum, to $95 \%$ of the top difference with respect to the minimum. Figure 2 shows a graphical representation of this thickness definition for wake-like defects, as presented in Papamoschou and Roshko (1988).

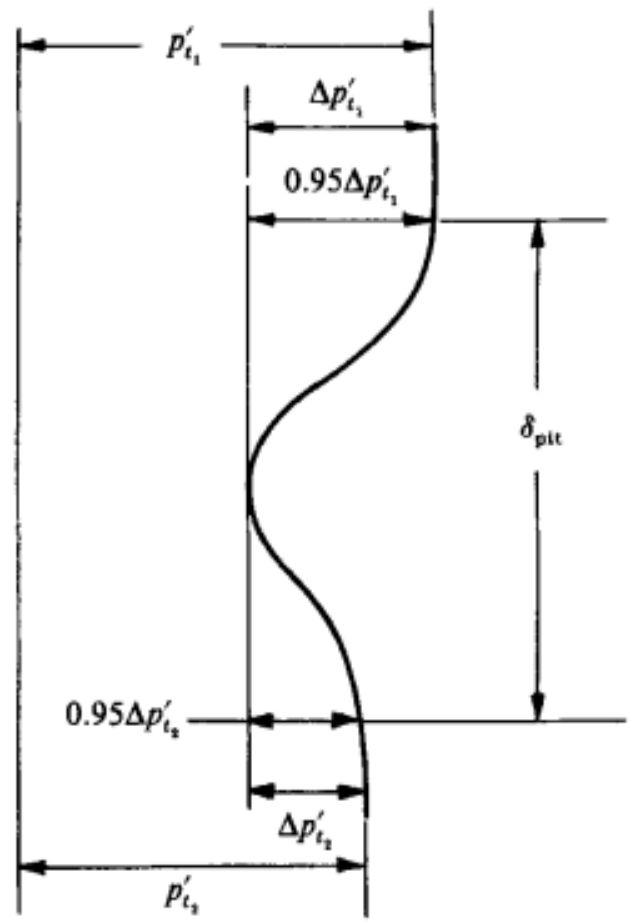

Figure 2. Graphical representation of Pitot thickness definition for wake-like defects. From Papamoschou and Roshko (1988).

\section{The Visual Thickness, $\boldsymbol{\delta}_{v i s}$}

The visual thickness is unlike the other definitions because it is not mathematically based. Rather, the visual thickness is commonly determined by drawing straight lines along the top and bottom edges of an image of the mixing layer and then calculating the growth rate based on those lines. Due to the inexact nature of this method, researchers often average the results from a large number of images taken during a brief time period.

The visual thickness has been suggested to be roughly twice the vorticity thickness (Brown and Roshko 1974), although the accuracy of this claim is obviously under question. 
Moreover, it is not likely that the visual thickness, which is primarily based on the density profile for shadowgraph and Schlieren images, would be directly relatable to the vorticity thickness, which is entirely based on the velocity profile.

\subsection{Incompressible Mixing Layer Velocity Profile Model}

The incompressible mixing layer was studied long before high speed mixing layers were considered, and as such, simple models for its velocity profile have been brought to a higher level of acceptance within the community. The two models discussed here, the error function velocity profile and the hyperbolic tangent velocity profile, are similar in form but achieve their sigmoidal shape through the use of different mathematical functions from

which they take their name. The error function profile, introduced by Schlichting (1979), is defined as

$$
\begin{gathered}
U^{*}=\frac{1}{2}\left[1+\operatorname{erf}\left(\frac{\sigma_{0} y}{\lambda_{s} x}\right)\right] \\
\lambda_{s}=\frac{(1-r)(1+\sqrt{s})}{2(1+r \sqrt{s})}
\end{gathered}
$$

The spreading parameter, $\sigma_{0}$, is an empirically tuned constant. A value of $\sigma_{0} \cong 11$ has been accepted for over six decades. Numerous suggestions ranging between $\sigma_{0}=10$ and $\sigma_{0}=12$ have been encountered (Liepmann \& Laufer 1947; Barone et al., 2006; Gatski \& Bonnet, 2009).

Dimotakis (1986) proposed a correction factor for spatial asymmetries. The modified mixing parameter, referred to here as $\lambda_{D 1986}$, is

$$
\lambda_{D 1986}=\frac{(1-r)}{2(1+r \sqrt{s})}\left[1+\sqrt{s}-\frac{1-\sqrt{s}}{1+2.9(1+r) /(1-r)}\right]
$$


This factor is claimed to apply a difference of up to $10 \%$ in extreme cases. Many authors neglect to consider this modified parameter and instead only use $\lambda_{s}$ (Gatski \& Bonnet 2009). Although it has been considered for the work presented here, the differences were insignificant. Therefore, $\lambda_{s}$ is used throughout this report for simplicity.

The hyperbolic tangent profile has been presented by Barone et al. (2006) in a very similar format to that of the error function profile, however it has been in use for many years before that (see Samimy \& Elliott 1990). The equation for the hyperbolic tangent profile can be written as

$$
U^{*}=\frac{1}{2}\left[1+\tanh \left(\frac{\sigma_{0} y}{\lambda_{s} x}\right)\right]
$$

Figure 3 illustrates a comparison between these two incompressible velocity profiles as well as their effect on the three velocity-based mixing layer thicknesses. It is apparent that for the same $\sigma_{0}$, the hyperbolic tangent profile predicts a larger mixing layer thickness for all three definitions. With proper tuning of $\sigma_{0}$ as applied to the hyperbolic tangent profile, these two functions can be made practically equivalent. To avoid this redundancy, only the more popular error function velocity profile will be carried forward in this report. 


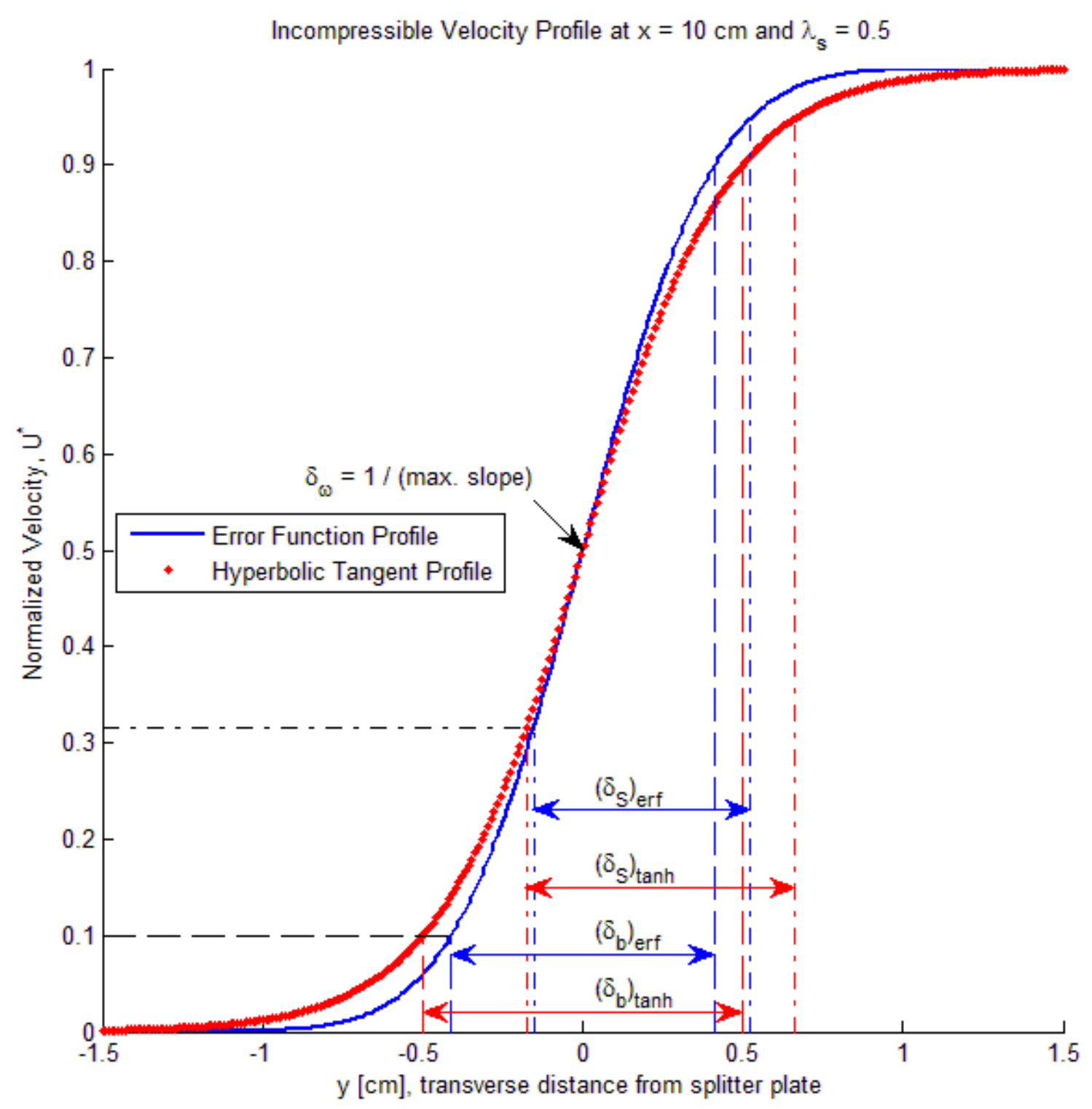

Figure 3. Comparison of the error function velocity profile and the hyperbolic tangent velocity profile, and their effect on velocity-based mixing layer thicknesses. In both cases, $\sigma_{0}=11.0$.

The behavior of the error function velocity profile is further illustrated in Figure 4 and Figure 5. In Figure 4, several streamwise instances of the profile are plotted to display the smoothing tendency of mixing layers. For low $x$-locations, the mixing layer is small in size and has a very sharp velocity gradient. Further downstream, the mixing layer is much thicker and has a lesser maximum velocity gradient. Figure 
5 demonstrates the self-similarity seen in the fully developed turbulent mixing layer.

By normalizing the transverse axis by the streamwise location (i.e. plotting $y / x$ on the horizontal axis), profiles measured at a variety of $x$-locations stack perfectly on top of each other. This plot also shows how increasing $\lambda_{s}$ leads to more rapid growth of the mixing layer.

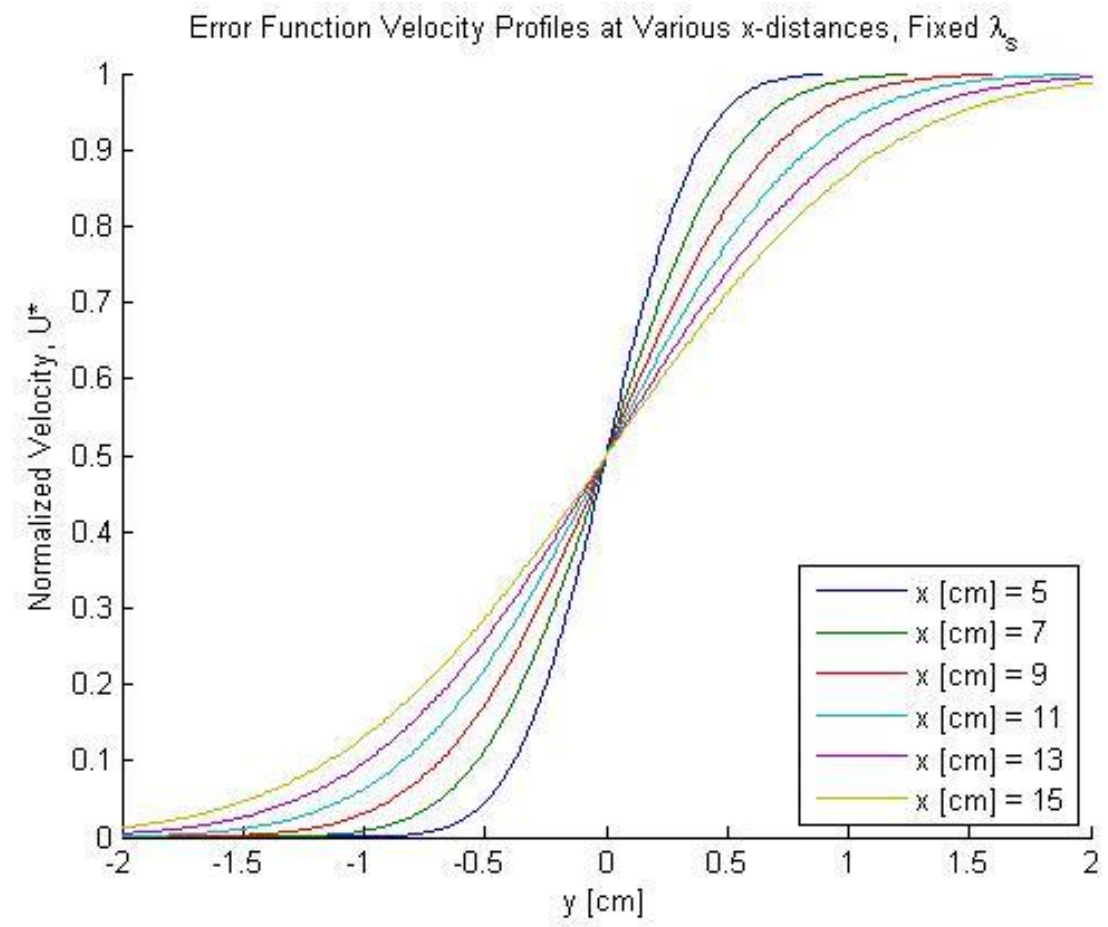

Figure 4. Error function velocity profile plotted for a fixed $\lambda_{s}$ over many $x$ locations. 


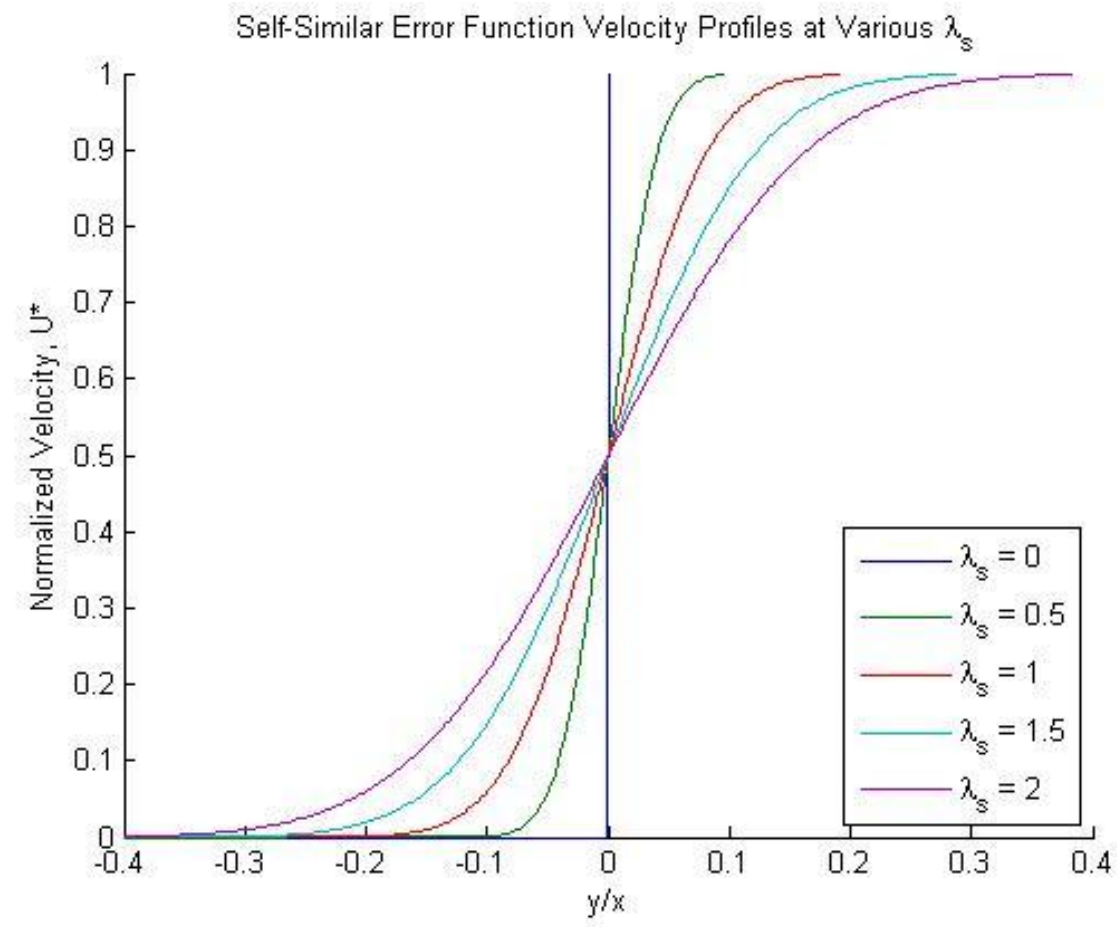

Figure 5. Self-similarity demonstrated in the error function velocity profile.

\subsection{Compressibility Effects on the Turbulent Mixing Layer Growth Rate}

Advanced aerospace propulsion technologies rely on rapid molecular mixing between drastically different streams. For instance, scramjets mix a subsonic aerosol fuel into a supersonic air stream in a very short distance. Alternately, air augmented rockets use a supersonic rocket plume to entrain subsonic ambient air. Although special mixing enhancement techniques are often utilized, the core phenomenon for all of these cases is the turbulent mixing layer.

\subsubsection{Observed Characteristics of Compressibility}

It has long been recognized that the introduction of compressibility into the turbulent mixing layer causes a significant reduction in mixing layer thickness growth rates (Maydew 
\& Reed 1963; Birch \& Eggers 1973; Brown \& Roshko 1974; Papamoschou \& Roshko 1988; Gatski \& Bonnet 2009). The incompressible, variable density experiment by Brown and Roshko (1974) proved that the growth rate reduction observed by other authors is separate from the effect of density ratio, indicating that compressibility itself is somehow independently associated with this phenomenon. It eventually took the application of direct numerical simulation (DNS) to determine that the reduced spreading is caused by a reduction in pressure fluctuations associated with higher compressibility (Vreman et al. 1996; Freund et al. 2000; Pantano \& Sarkar 2002). The growth rate reduction was shown to have an asymptotic limit at high compressibility because the pressure fluctuations could not diminish any further.

In order to better phenomenologically represent the mean scale effects of compressibility, Papamoschou and Roshko (1988) divided their experimentally determined growth rates by those predicted by an incompressible growth rate model using the same velocity and density ratios. In this way they created the normalized growth rate, defined as

$$
\phi=\frac{\delta^{\prime}}{\delta_{0}^{\prime}}=\frac{f\left(r, s, M_{c_{1}}\right)}{f\left(r, s, M_{c_{1}}=0\right)}
$$

where $M_{c_{1}}$ is the convective Mach number, which serves as a measure of mixing layer compressibility. A novel analysis of this parameter's derivation is presented in Section 3.2 (page 40).

\subsubsection{Measures of Compressibility}

A simple, laboratory-frame Mach number does sufficiently describe the magnitude of compressibility in the two-stream mixing layer because the turbulent interactions between the streams are dependent on the difference between the two streams. Two parameters 
have been suggested as measures of the compressibility of the mixing layer. They are the convective Mach number, $M_{c}$, and Slessor's compressibility parameter, $\Pi_{c}$.

The idea for the convective Mach number was originally published by Bogdanoff (1983), although it didn't gain its name or popularity until it was more fully derived by Papamoschou and Roshko (1988). The convective Mach number is defined as

$$
\begin{aligned}
& M_{c_{1}}=\frac{U_{1}-U_{c}}{a_{1}} \\
& \text { and } \quad M_{c_{2}}=\frac{U_{c}-U_{2}}{a_{2}}
\end{aligned}
$$

where $U_{c}$, the convective velocity, is the speed of the large, coherent turbulent structures, and $a$ is the acoustic speed. The subscripts " 1 " and " 2 " indicate which of the two streams is used as a reference. It can be shown that the two definitions are directly relatable (see Section 3.2 for a complete derivation with error analysis). For this reason, researchers typically only use the primary convective Mach number, and often show it without the "1" subscript as $M_{c}$.

Figure 6 shows a fairly modern collection of experimental data and models for normalized growth rates plotted against the convective Mach number. The curves are from the empirically fit "Langley" curve (see Birch \& Eggers 1973), a semi-empirical curve fit from Dimotakis (1991a) which is discussed in greater detail in Section 3.4 (page 54), and the amplification of the Kelvin-Helmholtz mode obtained from Day et al. (1998) from linear stability analysis. It is evident from this plot that the data is far too scattered for any of the models to fit well. 


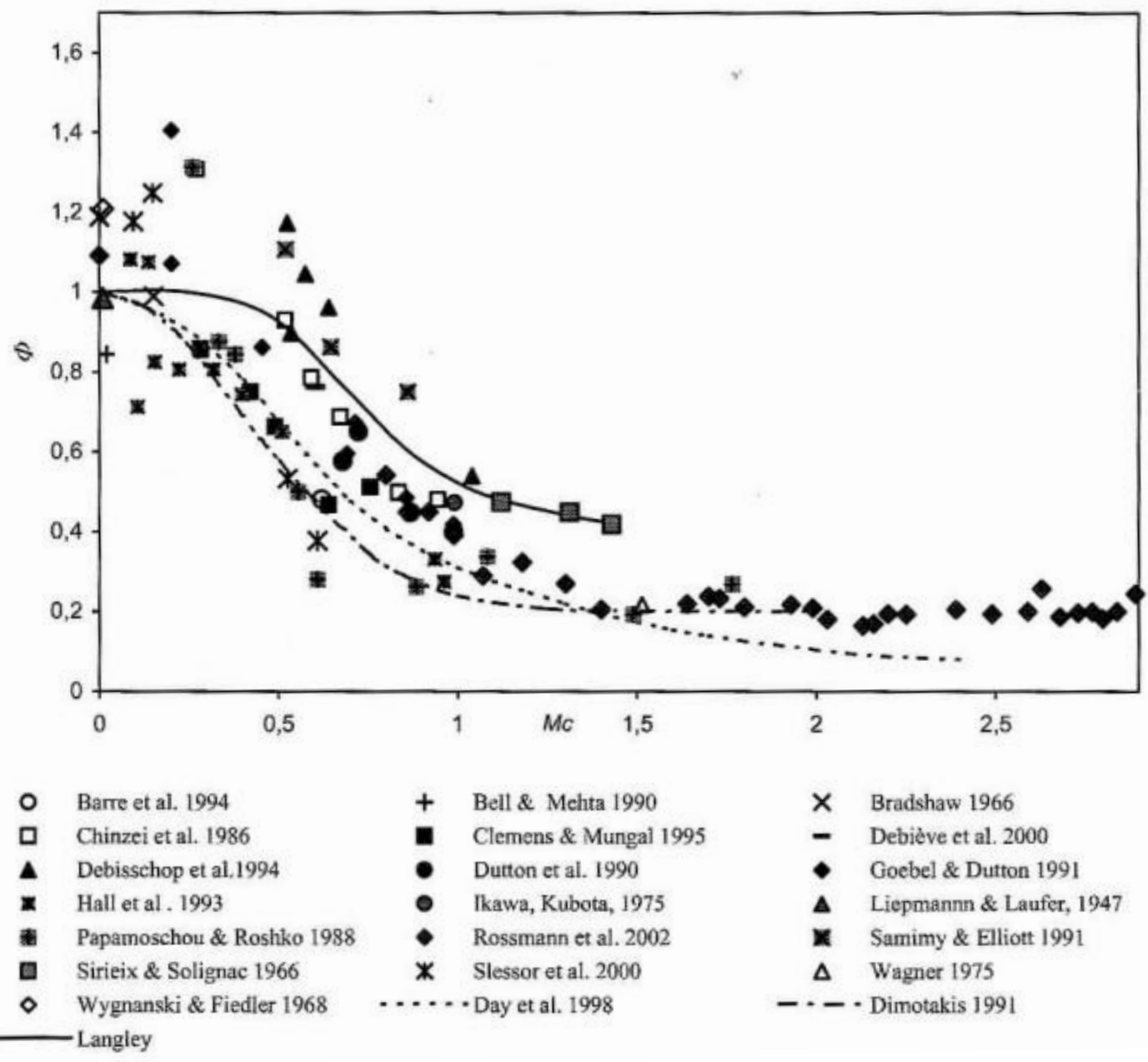

Figure 6. Normalized mixing layer thickness growth rates plotted against convective Mach number. From Smits and Dussauge (2006).

As a part of his doctorate thesis, Slessor (1998) introduced a maximum compressibility scale that was intended to address problems seen by the convective Mach number associated with extreme density ratios and acoustic speed ratios. This scale, referred to here as Slessor's compressibility parameter, is defined as

$$
\Pi_{c}=\max _{i=1,2}\left[\frac{\sqrt{\gamma_{i}-1}}{a_{i}}\right] * \Delta U
$$

where $i$ refers to the primary $(i=1)$ and secondary $(i=2)$ streams, respectively. 
Figure 7 shows this new parameter used in place of the convective Mach number, and the reduced scatter suggests a remarkable improvement. Curiously, this parameter has not gained much popularity among recent researchers. Section 3.1 (page 34) takes a deeper look at Slessor's contributions.

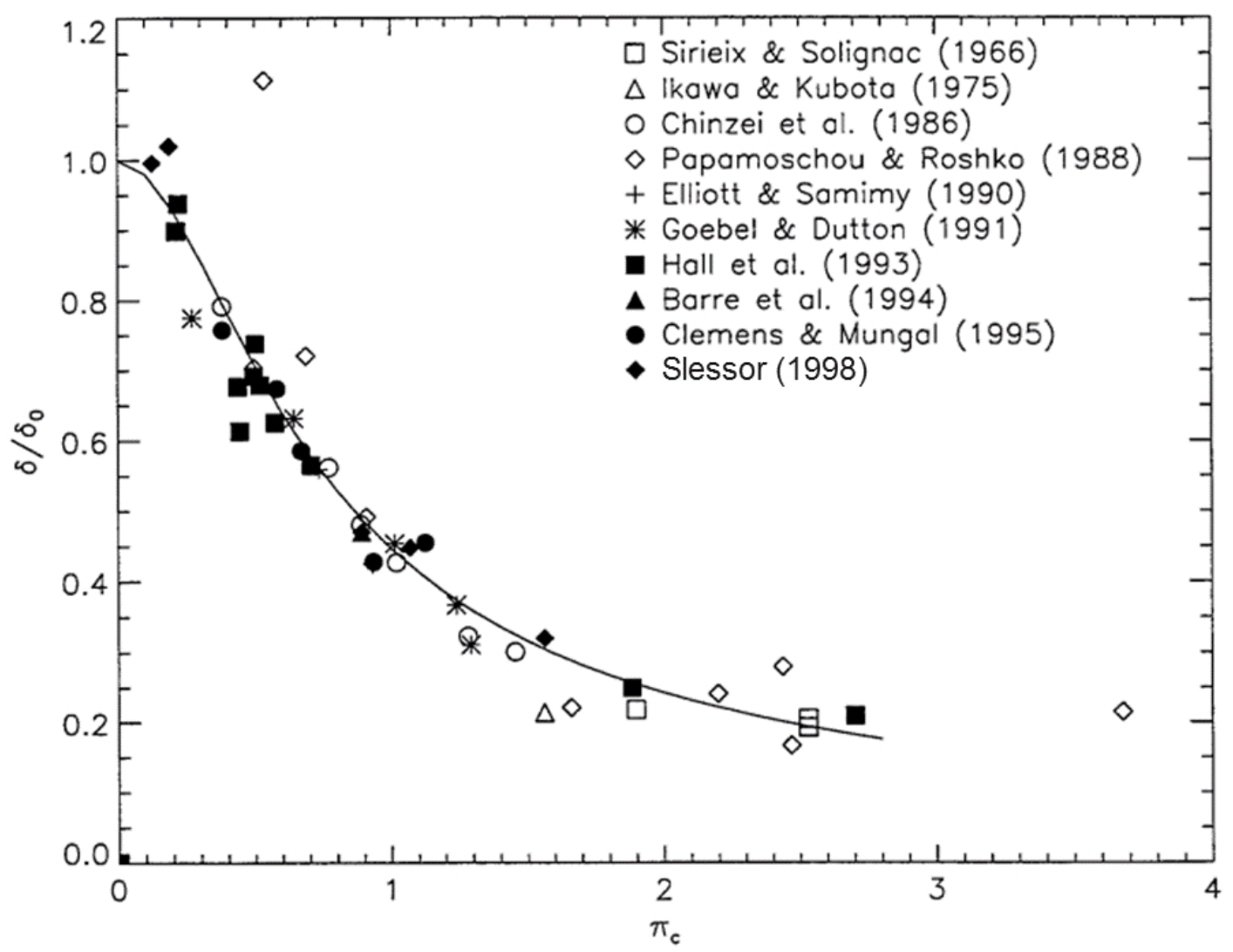

Figure 7. The application of Slessor's compressibility parameter to a collection of experimental growth rate data. Solid curve is a best-fit line. From Slessor (1998).

\subsection{Thesis Objectives}

The over-arching goal of this thesis is to improve upon existing models for predicting the growth rate of incompressible and compressible turbulent mixing layers. Section 2 discusses the creation of a contemporary database of experimental and 3D DNS mixing layer growth rate measurements gleaned from an exhaustive library of articles and reports 
related to turbulent mixing layers. Section 3 presents a deeper investigation into the existing models and various parameters that affect them in an effort to illuminate a preferred approach for establishing an improved model. Section 4 lays out the approach followed to develop the improved model, presents the model, and discusses its strengths and weaknesses. Section 5 offers concluding remarks about the work presented here and its significance on the global study of compressible turbulent mixing layers. The appendices offer a plethora of supporting documentation, including the database itself in tabular format and brief summaries of the articles that contributed viable data to the database. 


\section{The Database}

A collection of turbulent mixing layer test cases was derived from the literature and formed into the database shown in Appendix C. This database began as a library of references from which the data was extracted (see Appendix A), and it was further refined by a qualification rubric (see Appendix B). The turbulent mixing layer model and supporting analysis presented herein rely heavily on this database.

\subsection{Library of References}

A library of references relating to turbulent mixing layers was compiled prior to populating the database (see Appendix A). Unfortunately, there is no certain way to confirm absolute completion of this library. In fact, there is a very slim likelihood that every technical report, journal article, conference paper, textbook, and doctorate and master's thesis pertaining to turbulent mixing layers is identified in this library. The library does, however, offer a rather thorough collection of the literature, including all of the highly cited papers and many of the less popular ones.

Of the 174 references in the library, all but 12 were acquired and carefully inspected for applicable data (For one reason or another, the missing 12 papers could not be acquired by the available means.). The papers span over 70 years, with the oldest from 1942 and the newest from 2013. References were initially found through keyword searches on web databases. From there, new references were identified by tracking citation lineages both backward and forward in time. 


\subsection{Data Qualification Rubric}

A rubric was established to ensure that the data contributing to the database were reasonably similar to each other. Each metric within the rubric was intended to ensure that unwanted variables, known or suspected, did not contaminate the data set. This section introduces, clarifies, and justifies each item in the rubric. If any one of these items was not met by a test case, then the associated data was excluded from the present database. The reader is referred to Appendix B for rubric evaluations for all 162 papers (174 total minus 12 un-obtained).

\section{Qualification Metric \#1: The data must have come from either a laboratory experiment or three-dimensional DNS.}

Since this database was established to develop a more reliable model of the turbulent mixing layer, it would be undesirable to contaminate it with computation models that may incorrectly represent the physical phenomena. Therefore, RANS and LES numerical studies do not apply. For the same reason, theoretical predictions and trends, such as those from linear stability analysis (LSA), also do not apply. Furthermore, the prominently three-dimensional aspects of the compressible turbulent mixing layer indicate that twodimensional DNS would not suffice. Reichert and Biringen (2006) presented a side-byside comparison of two- and three-dimensional DNS computations that supports this claim.

\section{Qualification Metric \#2: Each case must be fully defined by a complete set of} parameters.

A complete parameter set includes:

1. Thickness definition type 
2. Observed mixing layer thickness growth rate, $\delta^{\prime}$

3. Primary gas (enough information to determine $\gamma_{1}$ and $R_{1}$ )

4. Secondary gas (enough information to determine $\gamma_{2}$ and $R_{2}$ )

5. Primary Mach number, $M_{1}$

6. Secondary Mach number, $M_{2}$

7. Velocity ratio, $r=U_{2} / U_{1}$

8. Density ratio, $s=\rho_{2} / \rho_{1}$

9. Total temperature ratio, $T_{t, 2} / T_{t, 1}$

The above parameters were used for calculating the convective Mach number, $M_{c}$, Slessor's compressibility parameter, $\Pi_{c}$, and a prediction for an incompressible growth rate of the same $r, s$, and thickness definition type, $\delta_{0}^{\prime}$. Also, they were used to confirm the assumption that static pressures are approximately constant (see Qualification Metric \#7).

It is possible to determine many of these parameters if enough additional information is provided. For instance, if $s$ was not explicitly defined, but $\delta_{0}^{\prime}$ and $r$ were, then $s$ can be reverse calculated. Another common example is if the normalized growth rate, $\phi$, was presented and the author either explicitly mentioned or alluded to a reference for the model used to determine $\delta_{0}^{\prime}$, then the observed growth rate could be determined.

\section{Qualification Metric \#3: The mixing layer must be planar.}

Axisymmetric mixing layers, commonly created from jet exhausts, experience an eventual collapse of their potential core, which is the region of uniform velocity within the center of the jet (see Freund et al. 1997). Downstream of the collapse of the potential core, the 
velocity at the centerline diminishes. This phenomenon makes the definition of an axisymmetric mixing layer thickness distinctly different from that of a planar mixing layer. Therefore, the study of axisymmetric mixing should remain separate from that of planar mixing.

\section{Qualification Metric \#4: The mixing layer thickness growth rate must be presented} in terms of its spatial development and not its temporal development.

The definitions and the measurement techniques of spatially developing and temporally developing mixing layer thickness growth rates are distinctly different. Because of these differences, it would be unsuitable to include both types within the same database. Although suggestions have been made for relating the two via the convective velocity, $U_{c}$, they are typically reliant on further assumptions and proportionality constants, which can introduce undesirable uncertainties (see Brown \& Roshko 1974).

\section{Qualification Metric \#5: The mixing layer must not be chemically reacting.}

Chemical reactions between the two streams of the mixing layer introduce a preponderance of additional phenomena that complicate the study of mixing layers. Although the study of fuel/oxidizer mixing and combustion is of great importance, these experiments and simulations are excluded from the present database, which focuses solely on the effects of turbulence and compressibility. Dimotakis (2005) offers a thorough, top-level discussion of the relationship between turbulent mixing and combustion.

\section{Qualification Metric \#6: The mixing layer must be free from artificial mixing} enhancement techniques.

Researchers have been actively searching for effective ways to enhance mixing rates ever since it was discovered that compressibility hinders the growth rate of mixing layers. Most 
applications that contain turbulent mixing layers, including fuel injection and noise reduction, stand to benefit from simple yet effective artificial mixing enhancement techniques. A variety of techniques have been studied, each of which has performance characteristics associated with it. The effects of these different techniques must be kept separate from the core behavior of the turbulent mixing layer, which is the focus of the present database. In papers where a baseline, non-enhanced mixing layer case was also presented, that single case was not excluded from the database due to this qualification metric. Some of the many artificial mixing enhancement techniques that are included in the present library are as follows:

- Counterflowing streams, where $r<0$ (Petrie et al. 1985; Strykowski et al. 1993, 1996; Alvi et al. 1995a, 1995b; Papamoschou 1995)

- Swirl (Naughton et al. 1997)

- Acoustic perturbations (Ramaswamy \& Loth 1996; Brummund \& Nuding 1997; Doty \& McLaughlin 2000)

- Artificially high turbulence intensity (Göebel \& Dutton 1991)

- Upstream duct or splitter plate deformations (Wygnanski \& Fiedler 1970; Island et al. 1998)

- Steep splitter plate angles (Aso et al. 2009)

\section{Qualification Metric \#7: The static pressure gradient must be approximately zero.}

The goal of this metric was to attempt to isolate turbulence as the singular acting effect within the mixing layer dynamics. Barre et al. (1997) presented a side-by-side comparison of isobaric and non-isobaric mixing layers that showed a measurable difference between the two cases. Because of these differences, non-isobaric cases are excluded from the 
present database. Cases were considered isobaric if the static pressure ratio, $P_{S_{2}} / P_{S_{1}}$, was between 0.9 and 1.1. Many researchers gave special consideration to ensuring compliance to this metric in the design of their apparatus. In cases where the static pressure ratio could not be determined (e.g. Chinzei et al. 1986), proof of such special consideration could qualify the case for the present database.

\section{Qualification Metric \#8: Bounding walls must be sufficiently far away from the mixing layer to avoid wall effects.}

If the edges of a mixing layer approach a bounding wall, pressure fluctuations and wall boundary layer effects can modify the growth rate of the mixing layer (Erdos et al., 1992). Therefore, cases that experienced this situation are excluded from the present database. The non-bounded mixing layer is often referred to as a free shear layer.

\section{Qualification Metric \#9: Measurements must be taken from a self-similar portion of} the mixing layer.

The mixing layer trait of self-similarity (discussed in Section 1.1) is used to ensure consistent measurement of constant mixing layer growth rates. If measurements are taken in the developing region of the mixing layer wherein growth rates are often more rapid, then the observed trends for the mixing layer thickness can be nonlinear and misrepresentative of the fully developed growth rate.

A variety of guidelines have been suggested for predicting the minimum downstream distance for self-similarity. Bradshaw (1966) claimed that the fully developed mixing layer can be found 1000 times the momentum-deficit thickness of the initial boundary layer downstream of the splitter plate. That is, fully developed turbulence exists for 


$$
\frac{x}{\theta_{1}}>1000
$$

However, the generic applicability of this claim comes into question because Bradshaw's results were based on single-stream, incompressible mixing layers with uniform density. For this reason, Papamoschou and Roshko (1988) argued that a more pertinent parameter would be

$$
\frac{x_{e f f}}{\theta_{1}}>500
$$

Eq. 18

where

$$
x_{e f f}=x(1-r)
$$

Eq. 19

It is important to remember that these are merely guidelines, and that the ultimate goal is simply to ensure that the measurements were taken from a fully developed portion of the mixing layer. Due to the variety of guidelines and the timeline of their introduction, any case that made a concerted effort to address this problem qualifies for the present database. The effort could be shown in a variety of ways, including explicit compliance with Eq. 17 or Eq. 18, graphical proof of self-similarity via velocity profiles and/or unarguably linear mixing layer thickness trends, and statements from the author that selfsimilarity was observed.

\section{Qualification Metric \#10: The paper must be of sufficient quality.}

To avoid making this metric highly subjective, "sufficient quality" refers to a bare minimum level of quality. That is, papers must be free of blatant errors. For example, data presented in multiple places within the paper must be consistent. If such errors are found within a paper, it can be impossible to tell which representation is correct. Furthermore, the credibility of other aspects of the paper is drawn into question when such errors exist. It is unfortunate that such a problem exists, as it applies to some rather well-renowned articles. 


\section{Qualification Metric \#11: The publication must be of sufficient quality.}

This metric ensures that the published data has been peer-reviewed. Acceptable forms of publication include (but are not limited to) articles published in peer-reviewed journals, conference papers for conferences with referees, and faculty approved doctorate and master's theses.

\subsection{Database Composition and Data Diversity}

The library presented here is quite diverse, including numerous experimental, numerical, and theoretical references. One hundred sixty-two (162) references were acquired for this study. The contributions of 7 of these 162 were clearly intended to be superseded by a later publication. Among the rest, 81 discussed some form of laboratory experiment. Of the references that did not discuss an experiment (papers that discussed an experiment in addition to some other investigation are bookkept here as experimental), 49 presented a numerical study. These numerical studies included 2D and 3D direct numerical simulations (DNS), large eddy simulations (LES), Reynolds-averaged Navier-Stokes computational fluid dynamics (RANS CFD), linear stability analysis (LSA), and probability density function (PDF) analysis. The remaining references are a smorgasbord of theoretical discussions, textbook chapters, topic reviews, and databases.

Although 49 different references presented a numerical study, only the singular case from Zhou et al. (2012) could be included in the present database because it was the only numerical study that simultaneously met Qualification Metrics \#1 (3D DNS) and \#4 (spatially developing mixing layer growth rates). The computational requirements for 3D DNS are innately expensive, so the additional requirement for steady state simulations has been prohibitively expensive until very recently. 
The data extracted from the library also exhibits good variety. Of the 78 viable data points, 49 were studies wherein the primary and secondary gases were of the same composition, while the other 29 cases were made from mixed gases. With regards to subsonic versus supersonic, 25 of the 78 cases mixed two subsonic streams, 46 mixed a subsonic stream with a supersonic stream, and 7 mixed two supersonic streams. The thickness definitions of the viable data points consisted of 41 velocity-based, 27 visual, and 10 density-based observations.

The histograms in Figure 8 through Figure 13 illustrate the diversity of the data contained in the database. Each bin contains values including the low end and not including the high end, except for the highest valued bin which also includes values at the high end. Overall, these show ample variety for many different parameters. The velocity ratio frequencies appear skewed toward the low end because cases where $r \rightarrow 1$ behave more as wake flows than as shear layers. The density ratios show good diversity shortly above and below unity, and the high values are also mildly represented with some extreme density ratio cases from Brown and Roshko (1974). The combination of the velocity and density ratios produce a well-represented variety of mixing parameters. The convective Mach number is skewed toward the low end due to the inherent difficulty associated with producing and accurately observing high $M_{c}$ cases. Immediate benefits could be seen with just a few cases in the $0.2<M_{c}<0.4$ and $1.0<M_{c}<1.2$ regions. The acoustic speed ratio, which is a combination of gas composition, total temperature ratio, and primary and secondary stream Mach numbers, is closely packed near unity with the addition of a handful of cases at extremely high values. 


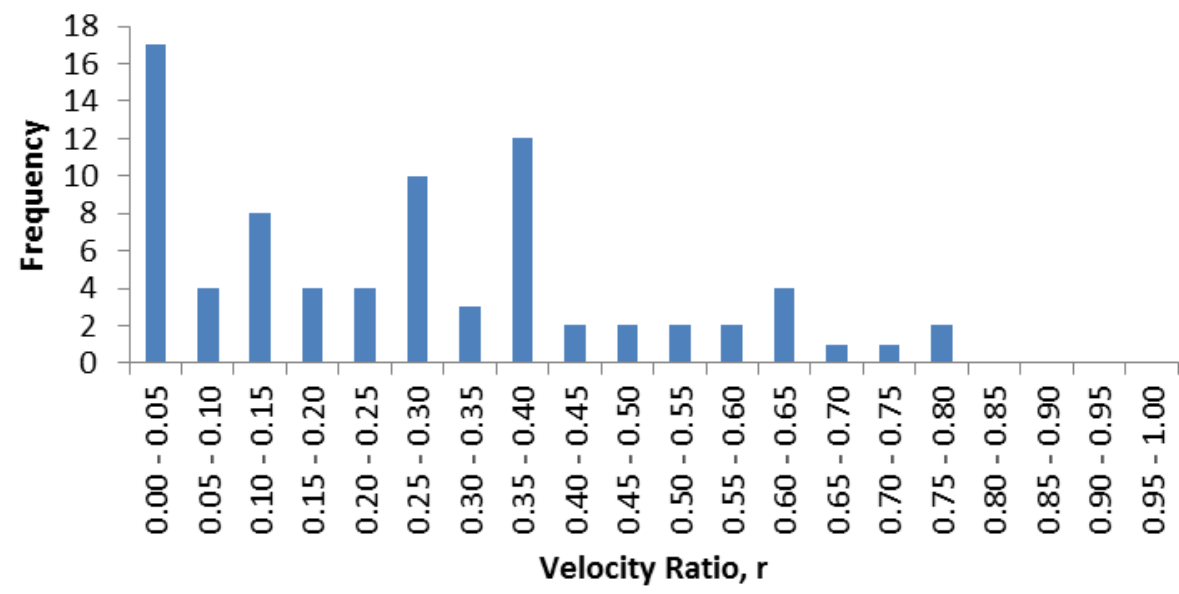

Figure 8. Histogram of velocity ratios within the database.

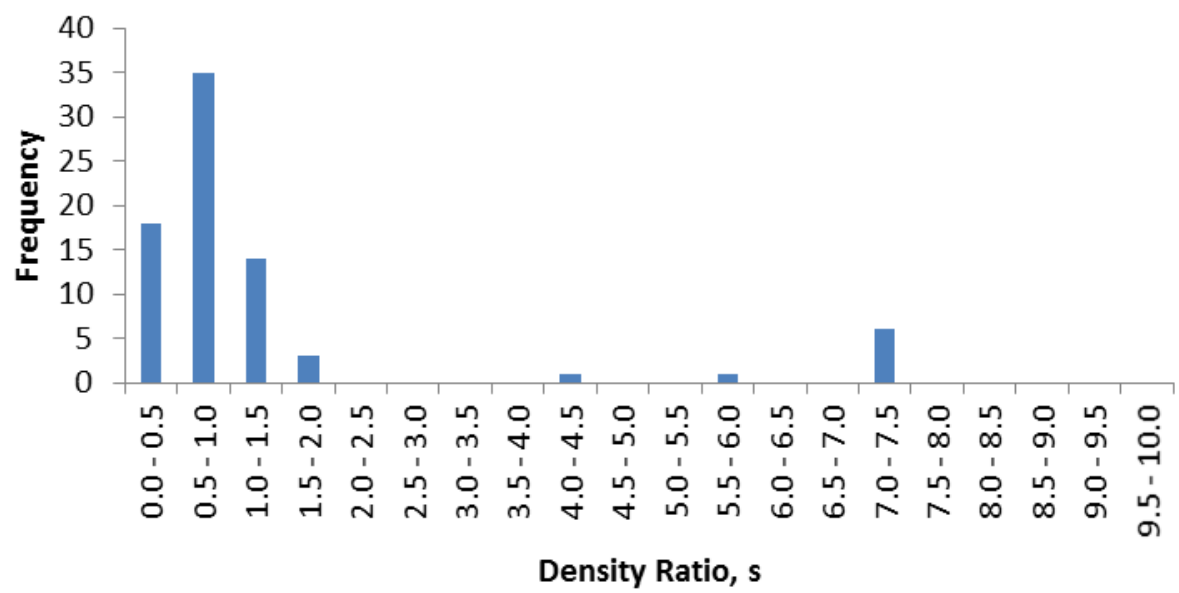

Figure 9. Histogram of density ratios within the database.

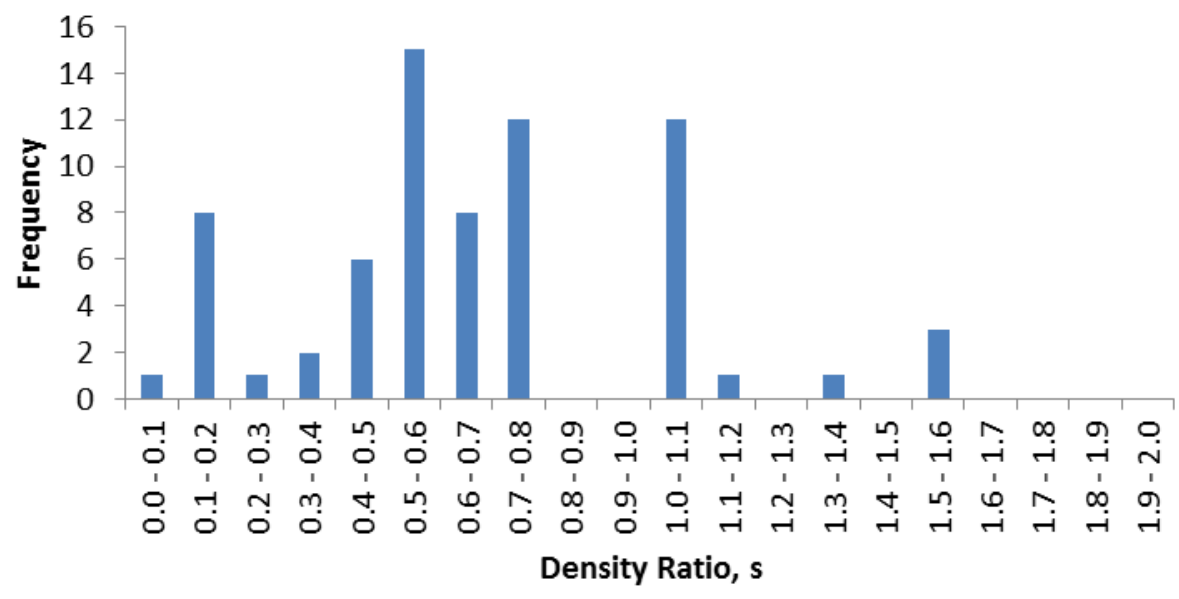

Figure 10. Close-up of Figure 9 for small density ratios. 


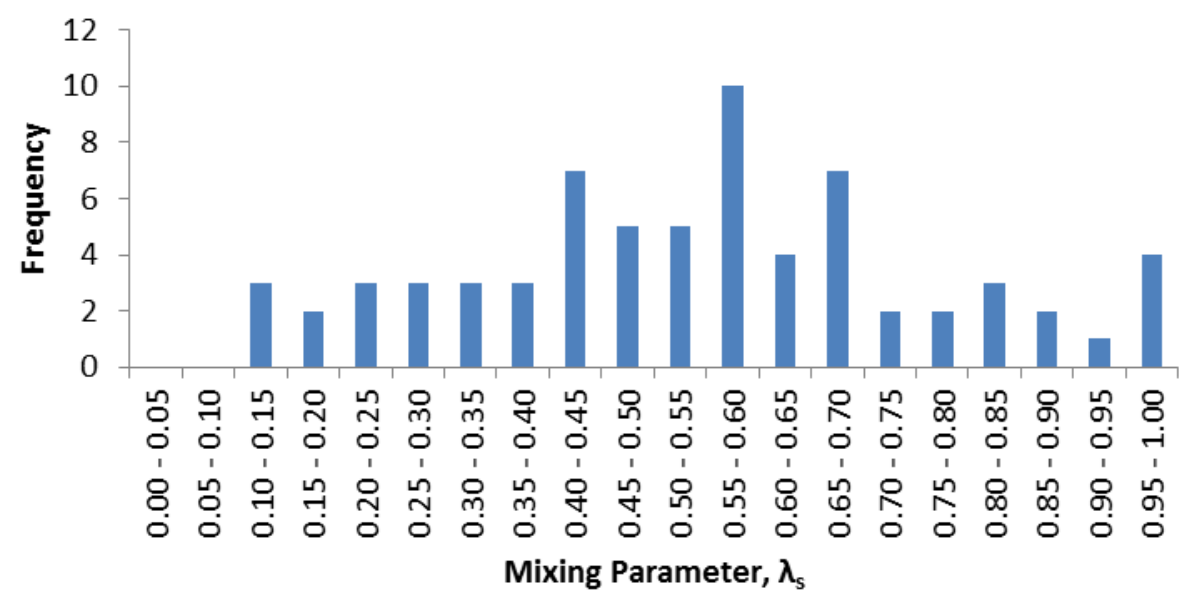

Figure 11. Histogram of mixing parameters within the database.

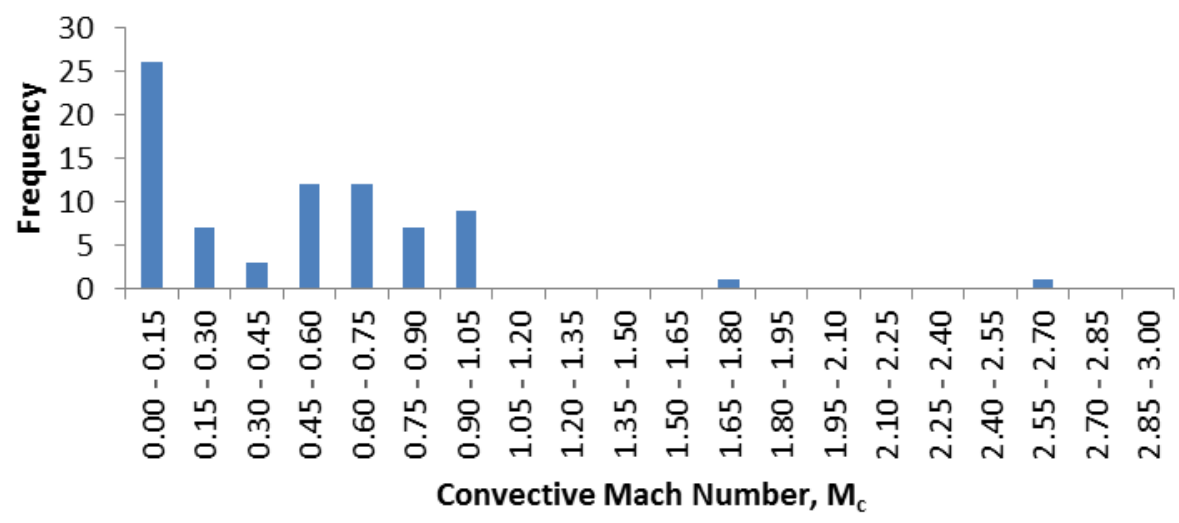

Figure 12. Histogram of convective Mach numbers within the database.

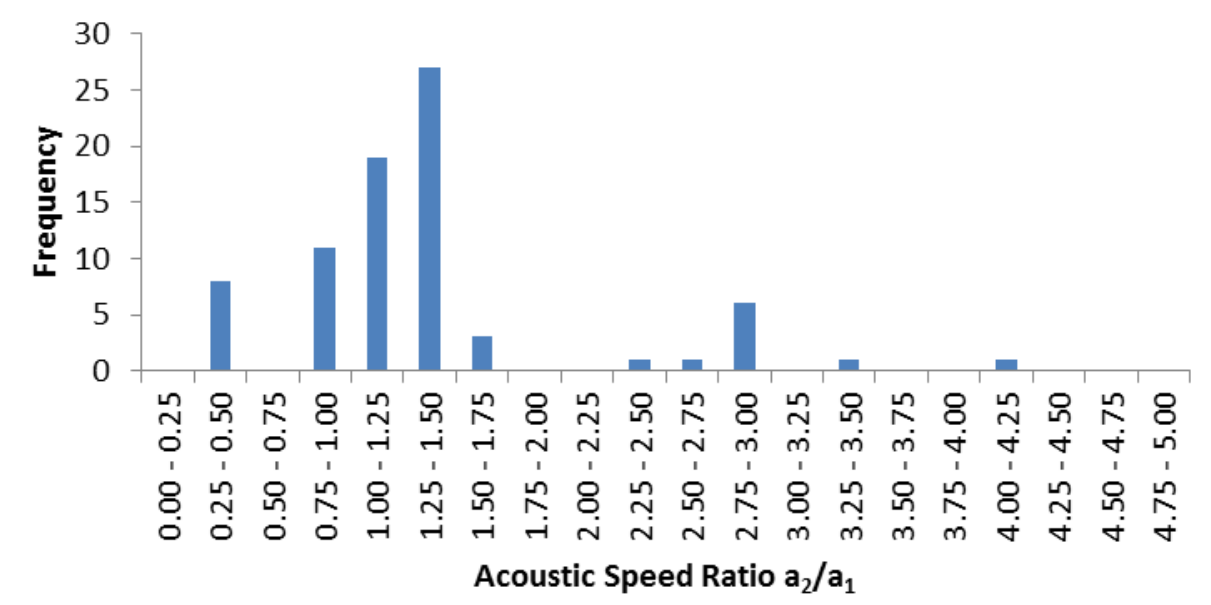

Figure 13. Histogram of acoustic speed ratios within the database. 
The collection of histograms shown here collectively present the diversity of cases within the database. Although this database does not exhibit ideal characteristics for trend fitting, it is sufficient for initial estimates. These histograms are presented with the hope that future researchers will use them as guidance for designing their cases.

\subsection{Discussion on Uncertainty}

As with all experiments and numerical studies, the cases within the present database are subject to uncertainty. In addition to the uncertainty reported by each author, an additional albeit small amount of uncertainty was introduced when the data was interpreted from the original publications. These sources are described in the following subsections.

\subsubsection{Reported Uncertainty within the Articles}

An attempt was made to collect information about the uncertainties associated with each data point. This attempt was met with varied successes. Only one out of the 41 velocitybased observations was presented with uncertainty information that was propagated to the growth rate level. Meanwhile, 18 out of the 27 visual growth rate data points and 3 out of the 10 density-based growth rate data points came with uncertainty information. Similarly, error bars could not be obtained for the convective Mach number or Slessor's compressibility parameter because the values were calculated from multiple parameters whose uncertainty was seldom reported. Overall, the lack of error bars in the literature is rather underwhelming.

It should be clarified that there were some authors who listed the uncertainties of their instruments but did not propagate those uncertainties through to the growth rate level. These authors presumably left the exercise of propagating errors for the interested reader. 
Unfortunately, the exact process followed during and after these experiments was seldom explained with enough detail for accurate production of such calculations. Therefore, the challenge of propagating other authors' uncertainties was not surmounted during the present effort.

The uncertainty data that were available suggest that, on average, visual growth rate measurements are the most prone to error, while the velocity- and density-based growth rate measurements are roughly equal in accuracy. This result notionally confirms a logical conclusion that the visual estimation of the edge of a mixing layer performed by hand will be less precise than the systematic determination of certain values in the instrumentally measured velocity or density profile. Further speculation might lead to the notion that experimentalists using this more subjective approach were more concerned with the evaluation of their uncertainties than those using the more objective approach.

Due to the general lack of uncertainty information within the present database, the information that was available is presented here only for the reader's reference. That is to say, no error analysis was performed during the modeling process in Section 4.

\subsubsection{Errors Introduced in the Collation of the Database}

A number of graphics throughout the library needed to be quantitatively interpreted because the data that they contained was not presented elsewhere in text format. An open source program called Plot Digitizer (version 2.6.2) was used to complete this task. The program was written by Joseph A. Huwaldt and Scott Steinhorst. More information about the program and an opportunity to download the latest version are available at http://plotdigitizer.sourceforge.net/. 
Like most plot digitizers, this program imports an image and then prompts the user to identify the endpoints of the horizontal and vertical axes. The plot is calibrated by pairing the plot's axes values with the pixel locations of the endpoints. Data points can be extracted and stored after calibration by clicking the cursor on the plot. Plot Digitizer 2.6.2 has a zoom feature that allows the user to carefully select locations to within fractions of a pixel, rendering the systematic error from the program itself practically negligible. Effort can be made to capture large screenshots of the plots so that the images retain high resolution, thus enabling utilization of Plot Digitizer's accuracy.

The primary source of error introduced through digitization was that of human error. Data markers in published articles by necessity have a certain size to them so that they can be visually identified. When digitizing, the assumption was made that the center of these markers identifies the exact location of the plotted data. Based on this, the accuracy of the digitized data depended upon the accuracy with which the centers of the data markers were selected. The error involved in this process was generally less than $\pm 1 \%$ of the scale of the plot being digitized. For the normalized growth rate versus convective Mach number plots, this typically indicated the introduction of an uncertainty of less than \pm 0.01 for the normalized growth rate. The digitized convective Mach number values were used to identify the data point by comparing to the values calculated from other mixing layer parameters, and therefore have no associated digitization uncertainty.

The references that were processed using Plot Digitizer are individually identified in Appendix D. Due to the typically small magnitude, the digitization error associated with these references is not included in the error bars presented throughout the report. 


\subsection{Unaltered Representation of the Database}

The database is represented in Figure 14 in terms of $\phi$ vs. $M_{c}$ and in Figure 15 in terms of $\phi$ vs. $\Pi_{c}$. Each data point was extracted directly from the primary author except for the cases summarized in the consortium by Birch and Eggers (1973). To avoid clutter within the plots, tabular representations of all parameters and their associated citations are included in Appendix C.

When available, the incompressible growth rate by which each data point was normalized has been taken from the source, either as an exact value or as a particular model being used. When those were not available, the most commonly used models were applied. For the visual thickness, Papamoschou and Roshko (1988) suggested that the incompressible growth rate could be determined as

$$
\delta_{0}^{\prime}=0.17 \lambda_{s}
$$

For the velocity-based thicknesses, the incompressible growth rates were estimated using the coefficients presented for the error function profile in Barone et al. (2006), which used $\sigma_{0}=11.0$. Unfortunately, since no such model has been presented for the momentum thickness, those cases could not be included in these graphs despite being viable entries in the database.

It can be seen that these plots do not conform well to the published models, although there is slight improvement over the scatter shown in Figure 6. It is suspected that this slight improvement is attributed to the careful application of the Data Qualification Rubric in Section 2.2. Section 3 further investigates reasons for this scatter. 


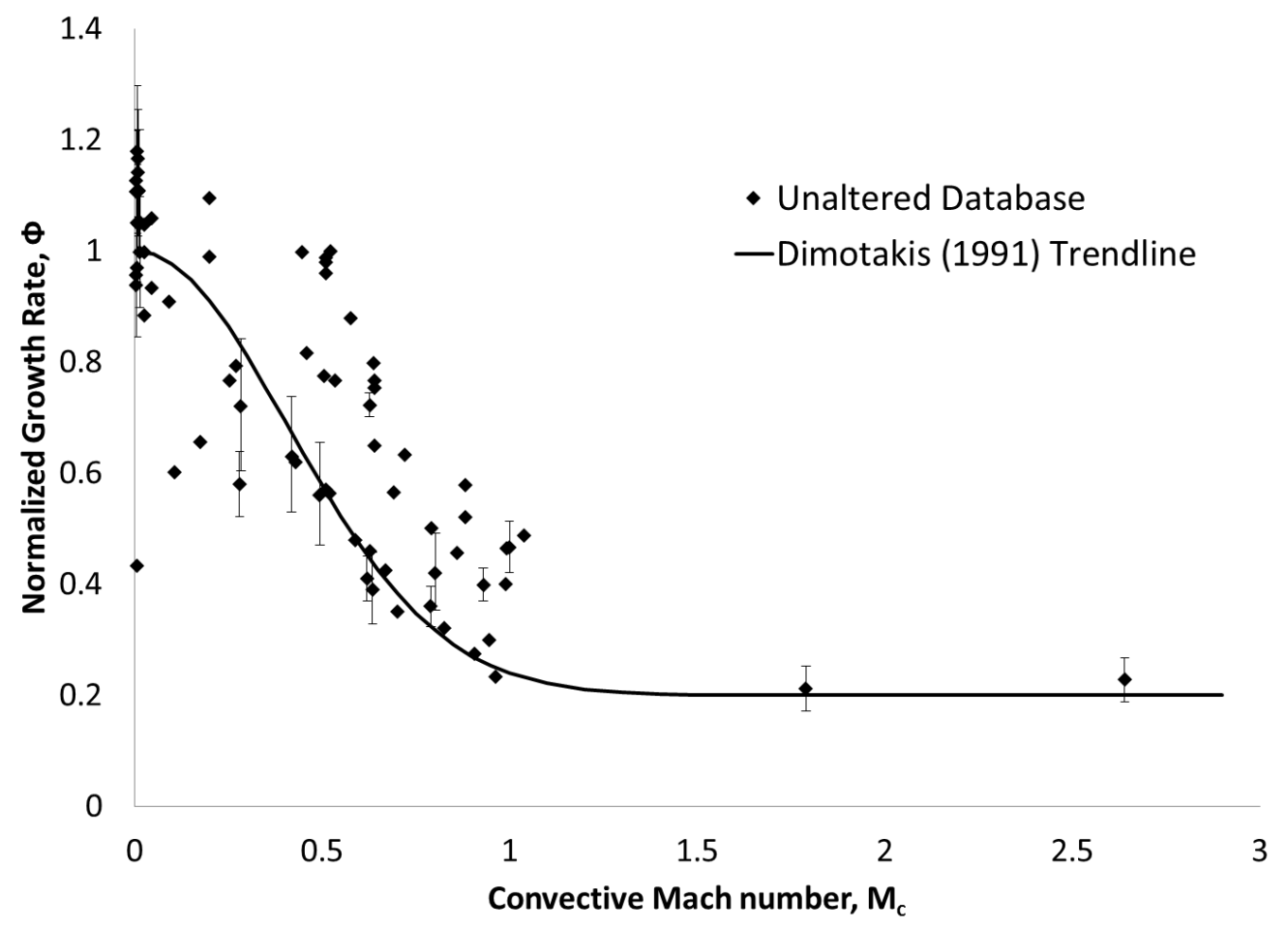

Figure 14. The unaltered mixing layer database represented in terms of convective Mach number.

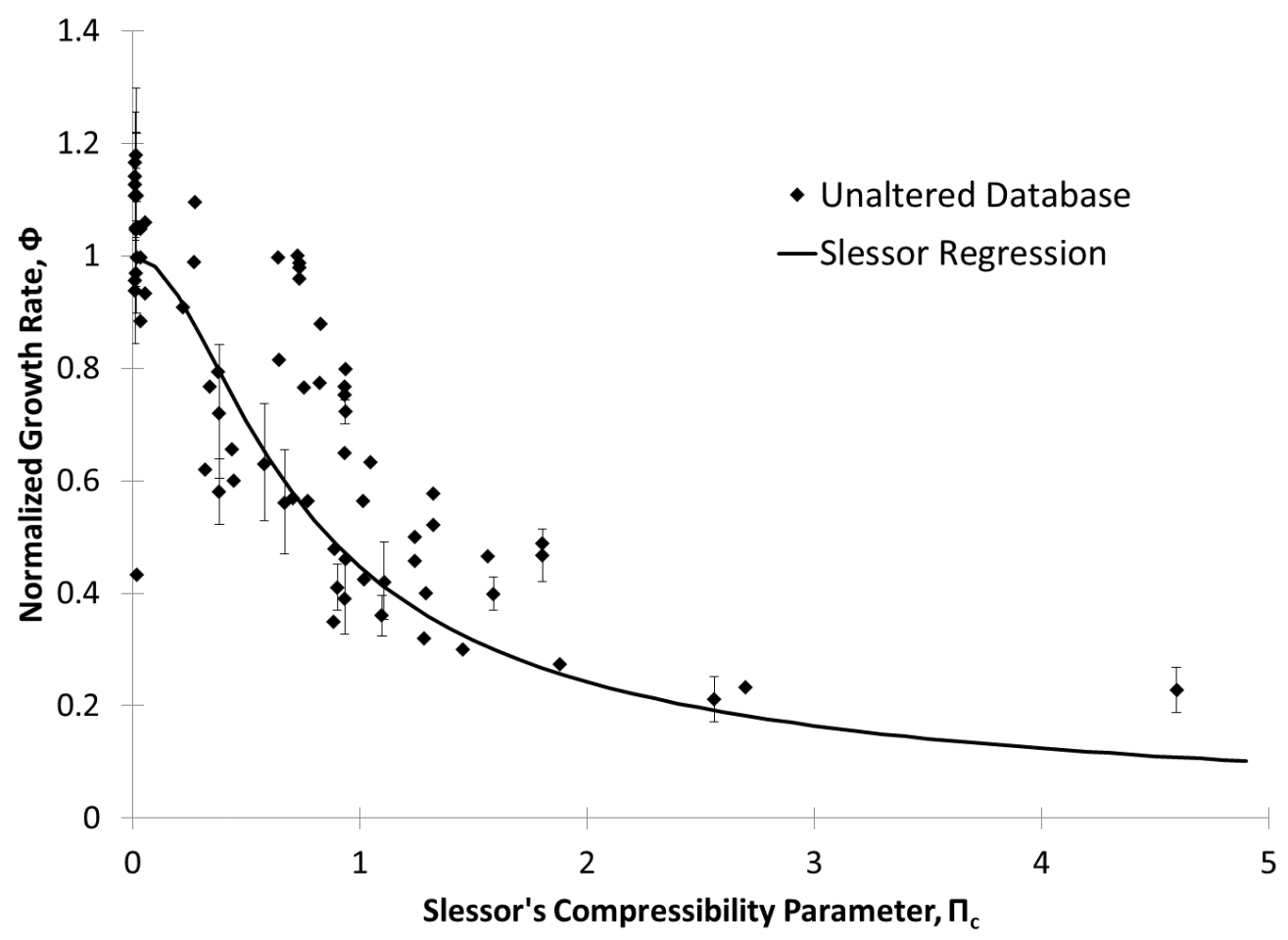

Figure 15. The unaltered mixing layer database represented in terms of Slessor's compressibility parameter. 


\section{Deeper Analysis of Existing Models}

A deeper analysis of the existing models was required to identify the most promising approach for improving the normalized growth rate prediction trends. To identify the potential areas for further investigation, consider the top-level definition of the normalized growth rate,

$$
\phi=\frac{\delta^{\prime}}{\delta_{0}^{\prime}}=f\left(M_{c} \text { or } \Pi_{c}\right)
$$

According to this equation, the variation can arise from among the three distinct sources described below.

1. The variable within the model function, $M_{c}$ or $\Pi_{c}$

2. The observed (experimental or numerical) growth rate, $\delta^{\prime}$

3. The incompressible growth rate model, $\delta_{0}^{\prime}$.

\section{Model Function, $M_{c}$ or $\Pi_{c}$}

Variance within the trend could have been introduced if the basis of the model function was inappropriate. Presently, the two parameters that have been suggested for this role are the convective Mach number, $M_{c}$, and Slessor's compressibility parameters, $\Pi_{c}$. These could be deemed inappropriate if their derivations introduce large amounts of uncertainty through the required assumptions or if they fail to capture a critical physical phenomenon. These parameters are scrutinized in Sections 3.1 and 3.2 to address the present concerns. 


\section{Observed Growth Rate, $\boldsymbol{\delta}^{\prime}$}

This source of scatter primarily has to do with the effect of experimental error. Section 2.4 discusses the general lack of uncertainty information within the present database. However, enough information was available to suggest that the typical experimental uncertainty is not large enough to singlehandedly account for the scatter within the data. Beyond the typical uncertainty, all experiments are prone to the possibility of outliers. Overall, it is unlikely that a large magnitude of experimental error has consistently affected the study of turbulent mixing layer growth rates from dozens of authors at many different facilities over decades of research.

\section{Incompressible Growth Rate Model, $\delta_{0}^{\prime}$}

The final possible source of scatter suggests that the normalization parameter is either incorrect, incomplete, or improperly used. Sections 3.3 and 4.3 show that the combination of these three fallacies within the incompressible growth rate model is largely responsible for the observed scatter in the compressibility trends and that they can all be resolved with proper care.

Section 1.2 introduces the error function velocity profile as the basis of the incompressible growth rate model. Section 3.3.1 presents a mathematical derivation of the incompressible growth rate coefficients based on the error function velocity profile. The results of this derivation offer more generic formulae for the coefficients than have previously been published, and these formulae match the coefficients from Barone et al. (2006) for $\sigma_{0}=$ 11.0. Further investigation of the tuning parameter in Section 4.3 suggests that a more optimal value can be found. 
The present incompressible growth rate model is a function of the mixing layer thickness definition, the velocity ratio, and the density ratio. Previous authors have noted that a variety of experimental nuances could play a significant role in the observed growth rate (see Gatski \& Bonnet 2009 for a more complete discussion). The qualification rubric discussion in 2.2 was used to eliminate many of these nuances from the present database. Section 3.3.2 identifies a further distinction that should be accounted for.

With the vast time span and author base through which the compressible mixing layer has been studied, it is very possible that the normalization parameter has not been applied in exactly the same way each time. Section 3.3 .3 shows that this possibility is an unfortunate reality.

\subsection{Slessor's Contribution, Revisited}

Slessor (1998) attempted to address the scatter in the existing normalized growth rate trends in Chapter 6 of his doctorate thesis. Through that chapter, he introduced a method for re-normalizing the observed growth rate and then presented a novel compressibility scale. These two contributions are revisited here.

\subsubsection{Evaluation of Slessor's Re-Normalization Technique}

The starting point for Slessor's investigations is shown in Figure 16. It contains a modest collection of the highly cited articles on compressible turbulent mixing layers. 


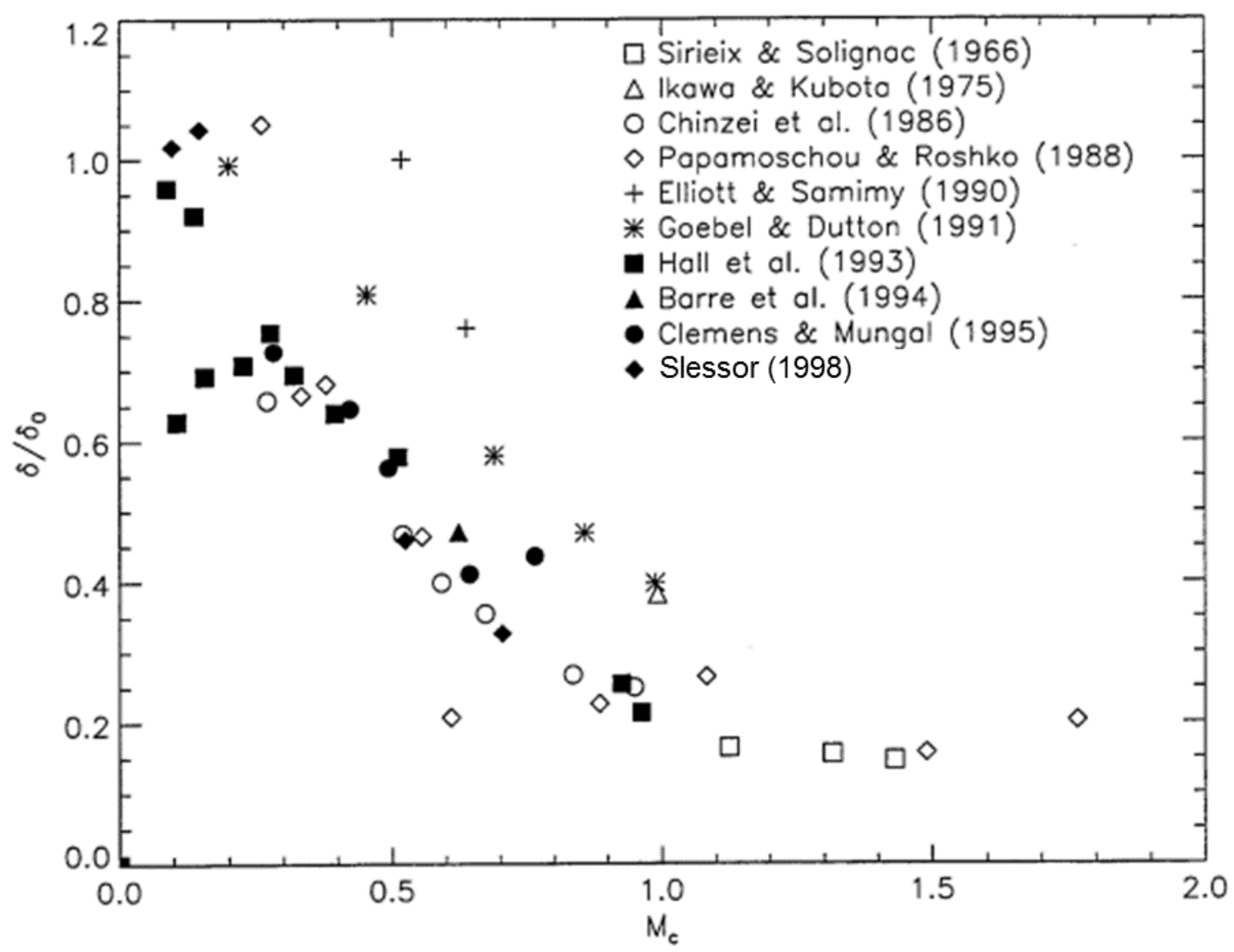

Figure 16. Slessor's initial data set, reproduced from Figure 6.1a of his 1998 thesis.

Slessor noted that the scaling within individual sets of experimental data seems rather consistent despite the overall scatter in the trend. For this reason, he recommended adjusting the normalization parameter to account for the deviation caused by the use of different experimental facilities. The process he used to do this is listed below.

1. Choose a single case from each researcher's data set to serve as a reference. It was deemed preferential to use the case nearest to $M_{c}=0.5$ because most researchers of compressible turbulent mixing layers had a case that approached 
that value. The convective Mach number of the chosen case is named $M_{c, r e f}$, the reference convective Mach number.

2. Using $r$ and $s$ from the reference case, apply the following equation from Dimotakis (1986) (See Section 1.2):

$$
\frac{\delta_{0}^{\prime}}{C_{\delta}}=\frac{(1-r)(1+\sqrt{s})}{2(1+r \sqrt{s})}\left[1-\frac{(1-\sqrt{s}) /(1+\sqrt{s})}{1+2.9(1+r) /(1-r)}\right]
$$

3. Next, evaluate the following model (Dimotakis 1991a) using $M_{c, r e f}$ from step 1.

$$
\left(\frac{\delta^{\prime}}{\delta_{0}^{\prime}}\right)_{\text {trend }}=0.8 e^{-3 M_{c}^{2}}+0.2
$$

4. Finally, the new incompressible growth rate model coefficients are determined by the following relationship:

$$
C_{\delta}=\left(\delta^{\prime}\right)_{o b s}\left(\frac{\delta_{0}^{\prime}}{\delta^{\prime}}\right)_{\text {trend }}\left(\frac{C_{\delta}}{\delta_{0}^{\prime}}\right)
$$

This approach was used to significantly reduce the scatter in the data set, as is shown in Figure 17. 


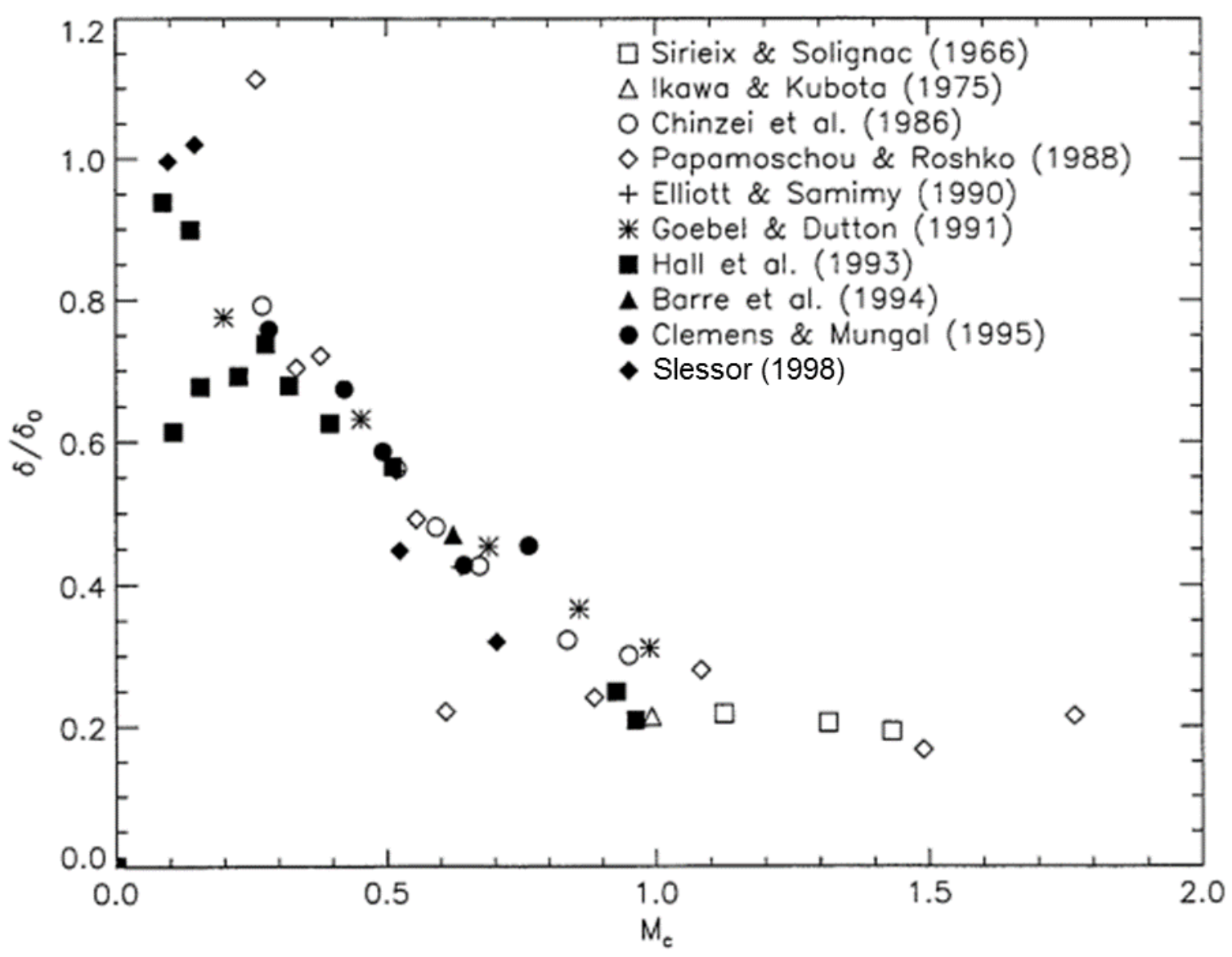

Figure 17. Slessor's re-normalized data set, reproduced from Figure $6.1 \mathrm{~b}$ of his 1998 thesis.

The above process is reliant on a particular assumption. To properly reduce terms from Eq. 24, one must assume that $\delta_{o b s}^{\prime}=\delta_{\text {trend }}^{\prime}$ at $M_{c, r e f}$. This approach artificially forces all of the data to collapse onto the Dimotakis (1991a) model near $M_{c}=0.5$, allowing the remainder of the data to follow suit. Three criticisms are made of this assumption:

1. It suggests that a vast majority of the scatter within the data was caused by differences in the apparatus without citing nor distinguishing the particular reasons for those differences. Such an approach precludes the possibility of applying the resulting trend to practical applications which would inevitably have their own unique environmental differences. 
2. It relies heavily on the heuristic trend of Dimotakis (1991a), which was based on the data available at the time. Although a wealth of applicable data was published in the years between Dimotakis' and Slessor's publications, no attempt was made to modernize or update the trend.

3. Entirely different results could be obtained if an alternate reference Mach number was chosen. Slessor's selection of $M_{c, r e f}=0.5$ was founded on the availability of data rather than physical justification. Unfortunately, Figure 16 shows that the greatest variance in the unaltered data occurs near $M_{c}=0.5$. While this coincidence maximizes the reduction in scatter, it emphasizes the point that the process would not have been as effective if a different $M_{c, r e f}$ were chosen.

Citing these criticisms, the assumption relied upon by Slessor's re-normalization approach is not appropriate for the post-processing of compressible turbulent mixing layer growth rate data.

\subsubsection{Evaluation of Slessor's Compressibility Parameter}

Slessor's compressibility parameter was derived from the notion that compressibility acts to couple kinetic and thermal energy (see Slessor 1998). A maximum compressibility scale can be derived by joining the ratio of kinetic to thermal energy (enthalpy),

$$
\frac{U^{2}}{2 h}=\frac{\gamma-1}{2} M^{2}
$$

with the freestream velocity difference $(\Delta U)$. This scale, here referred to as Slessor's compressibility parameter, is defined as

$$
\Pi_{c}=\max _{i=1,2}\left[\frac{\sqrt{\gamma_{i}-1}}{a_{i}}\right] * \Delta U
$$

or explicitly in terms of freestream values, 


$$
\Pi_{c}=\max \left[\sqrt{\gamma_{1}-1}\left(M_{1}-\frac{a_{2}}{a_{1}} M_{2}\right), \sqrt{\gamma_{2}-1}\left(\frac{a_{1}}{a_{2}} M_{1}-M_{2}\right)\right]
$$

Slessor proposed that the systematic deviations in flows with extreme density and/or speed-of-sound ratios that he observed when using the convective Mach number as an ansatz were largely accounted for with this new parameter. Finally, Slessor offered a fitted smooth curve through the data (which had already been altered by his revised $\delta_{0}^{\prime}$ ) to be

$$
\frac{\delta^{\prime}}{\delta_{0}^{\prime}}=\left(1+4 \Pi_{c}^{2}\right)^{-0.5}
$$

The combination of Slessor's re-normalization and his new compressibility parameter resulted in the reduced deviation shown in Figure 18.

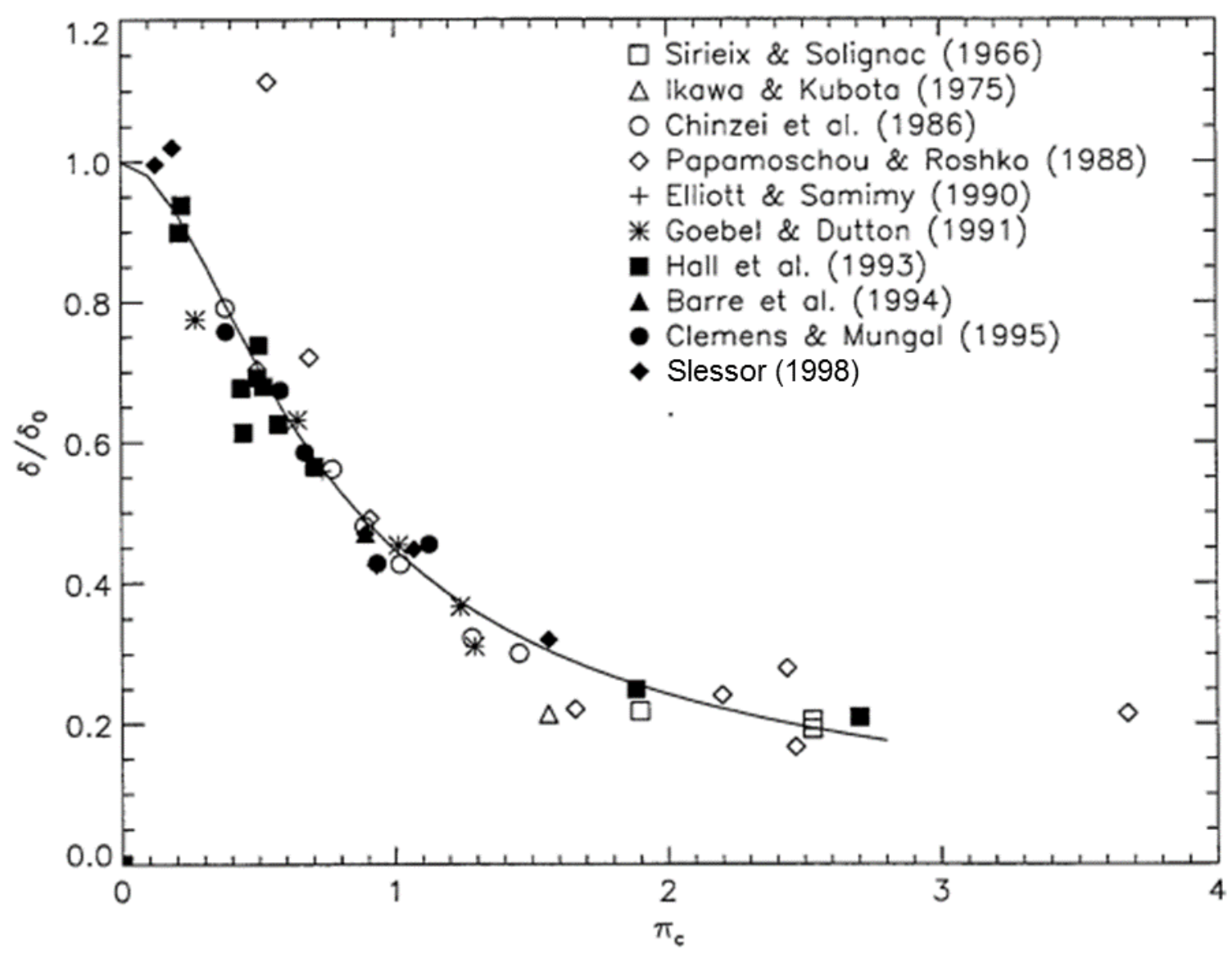

Figure 18. Slessor's re-normalized data set plotted against his compressibility parameter, reproduced from Figure 6.4 of his 1998 thesis. 
Although the trend in Figure 18 appears to present a marked improvement, it is no longer applicable after the discussion in Section 3.1.1. A more appropriate evaluation of the merits of Slessor's compressibility parameter is shown by comparing Figure 14 ( $\phi$ vs. $M_{c}$ ) and Figure 15 ( $\phi$ vs. $\Pi_{c}$ ) in Section 2.5 because the data being fit is equivalent between the two plots. This comparison shows only a minor decrease in variation affected by a transition from $M_{c}$ to $\Pi_{c}$. It is possible that Slessor's compressibility parameter may be applicable in future works, however the meager differences that are presently observed do not yet warrant a break from the widely accepted convective Mach number.

\subsection{The Convective Mach Number, Revisited}

The convective Mach number was originally suggested by Bogdanoff (1983), but it did not gain its popularity or its name until it was more fully derived by Papamoschou and Roshko (1988). Their derivation, which is reproduced here, covered the necessary steps for a reader to follow. However, it did not include a careful analysis of the assumptions and simplifications that were associated with the derivation. Such a careful analysis is included in this section.

The derivation began with an inspired perspective of the mixing layer. Rather than the typical laboratory frame of reference whereby the splitter plate is stationary and the large turbulent structures move at a convective speed of $U_{c}$, consider the streamlines as they would be shaped when the frame of reference is traveling downstream at speed $U_{c}$. Figure 19 demonstrates these two points of view. 


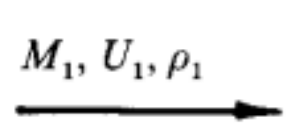

(a)
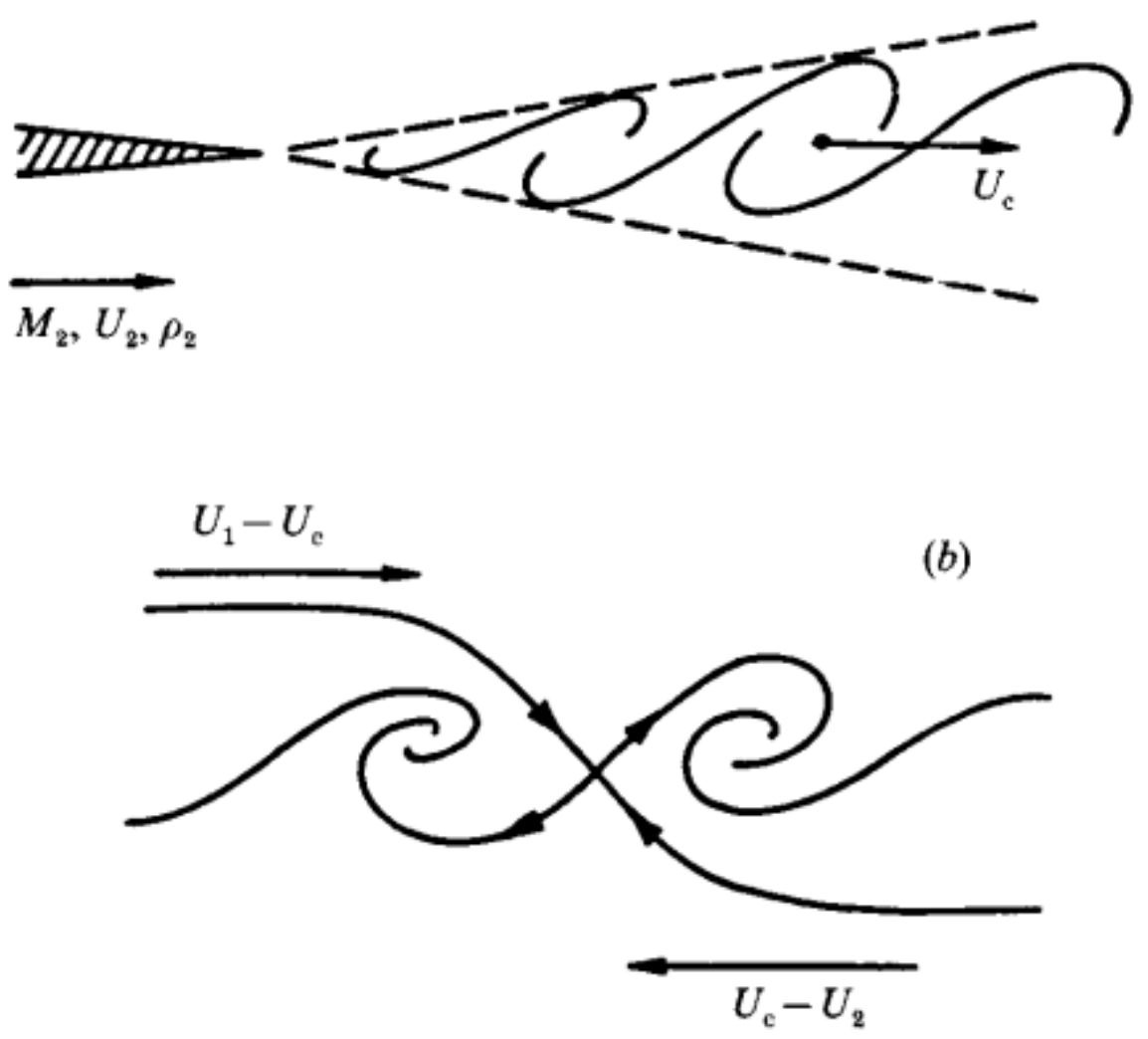

Figure 19. Sketches of streamlines of a turbulent mixing layer for (a) stationary frame of reference, and (b) convective frame of reference. From Papamoschou and Roshko (1988).

From the convective frame of reference, the Mach numbers of the primary and secondary streams are logically defined as

$$
\begin{aligned}
M_{c_{1}} & =\frac{U_{1}-U_{c}}{a_{1}} \\
\text { and } \quad M_{c_{2}} & =\frac{U_{c}-U_{2}}{a_{2}}
\end{aligned}
$$

This provides the definitions of the primary and secondary convective Mach numbers, respectively. With the new frame of reference in mind, the following three assumptions are made: 
1. Assume steady flow $(\partial / \partial t=0)$

2. Assume the saddle point between two adjacent turbulent structures is a common stagnation point $\left(P_{t_{2}}=P_{t_{1}}\right)$

3. Assume equal static pressures $\left(P_{s_{2}}=P_{s_{1}}\right)$

These three assumptions are fairly simple to justify or check. Steady flow is a trait that can be reproduced in a well-designed experiment. Although it is likely untrue for most practical applications, the assumption of steady flow is necessary for any remotely simple derivation. The shared stagnation point is easy to recognize when envisioning the streamlines as shown in Figure 19(b). The third assumption is quite easy to break in any practical setting, however it is well enough maintained in experimental settings. Metric \#7 of the qualification rubric for the present database ensured that this assumption was enforced to within $\pm 10 \%$. By accepting the above assumptions, the two streams can be related by

$$
\frac{P_{t_{1}}}{P_{S_{1}}}=\frac{P_{t_{2}}}{P_{S_{2}}}
$$

or in terms of isentropic flow relations,

$$
\left(1+\frac{\gamma_{1}-1}{2} M_{c_{1}}^{2}\right)^{\frac{\gamma_{1}}{\gamma_{1}-1}}=\left(1+\frac{\gamma_{2}-1}{2} M_{c_{2}}^{2}\right)^{\frac{\gamma_{2}}{\gamma_{2}-1}}
$$

Papamoschou and Roshko claimed that Eq. 32 could be simplified if $M_{c_{1}}$ is small and $\gamma_{2} \approx$ $\gamma_{1}$, resulting in

$$
M_{c_{2}}=\left(\frac{\gamma_{1}}{\gamma_{2}}\right)^{\frac{1}{2}} M_{c_{1}}
$$

What they did not mention is the justifying mathematics nor the error propagation associated with this simplification. These missing steps are described below. To begin, Eq. 32 is rearranged without simplification to form 


$$
M_{c_{2}}=\sqrt{\frac{2}{\gamma_{2}-1}\left[\left(1+\frac{\gamma_{1}-1}{2} M_{c_{1}}^{2}\right)^{\frac{\gamma_{1} \gamma_{2}-\gamma_{1}}{\gamma_{1} \gamma_{2}-\gamma_{2}}}-1\right]}
$$

Binomial expansion of the term in parentheses in Eq. 34 gives

$$
\begin{aligned}
\left(1+\frac{\gamma_{1}-1}{2} M_{c_{1}}^{2}\right)^{\frac{\gamma_{1} \gamma_{2}-\gamma_{1}}{\gamma_{1} \gamma_{2}-\gamma_{2}}} & \\
& \approx 1+\left(\frac{\gamma_{1} \gamma_{2}-\gamma_{1}}{\gamma_{1} \gamma_{2}-\gamma_{2}}\right)\left(\frac{\gamma_{1}-1}{2}\right) M_{c_{1}}^{2} \\
& +\frac{\left(\frac{\gamma_{1} \gamma_{2}-\gamma_{1}}{\gamma_{1} \gamma_{2}-\gamma_{2}}\right)\left(\frac{\gamma_{1} \gamma_{2}-\gamma_{1}}{\gamma_{1} \gamma_{2}-\gamma_{2}}-1\right)}{2}\left(\frac{\gamma_{1}-1}{2}\right)^{2} M_{c_{1}}^{4}+\cdots
\end{aligned}
$$

If $M_{c_{1}}$ is small and $\gamma_{2} \approx \gamma_{1}$, then the high order terms (i.e. $\left.\mathrm{O}\left(M_{c_{1}}^{4}\right)\right)$ can be neglected. This results in

$$
M_{c_{2}}=\sqrt{\frac{2}{\gamma_{2}-1}\left[1+\left(\frac{\gamma_{1} \gamma_{2}-\gamma_{1}}{\gamma_{1} \gamma_{2}-\gamma_{2}}\right)\left(\frac{\gamma_{1}-1}{2}\right) M_{c_{1}}^{2}-1\right]}
$$

which can be reduced without further assumptions to Eq. 33. The error associated with this simplification is graphically depicted in Figure 20, which shows the percent difference between Eq. 33 and Eq. 34 for typically found values of $M_{c_{1}}$ and $\gamma_{2} / \gamma_{1}$. Note that there is zero error when $\gamma_{2}=\gamma_{1}$ and that the error remains low when $M_{c_{1}}$ is small (i.e. $<1$ ) regardless of $\gamma_{2} / \gamma_{1}$. The error becomes significant when $M_{c_{1}}$ is above 1.5 and $\gamma_{2} \neq \gamma_{1}$. 


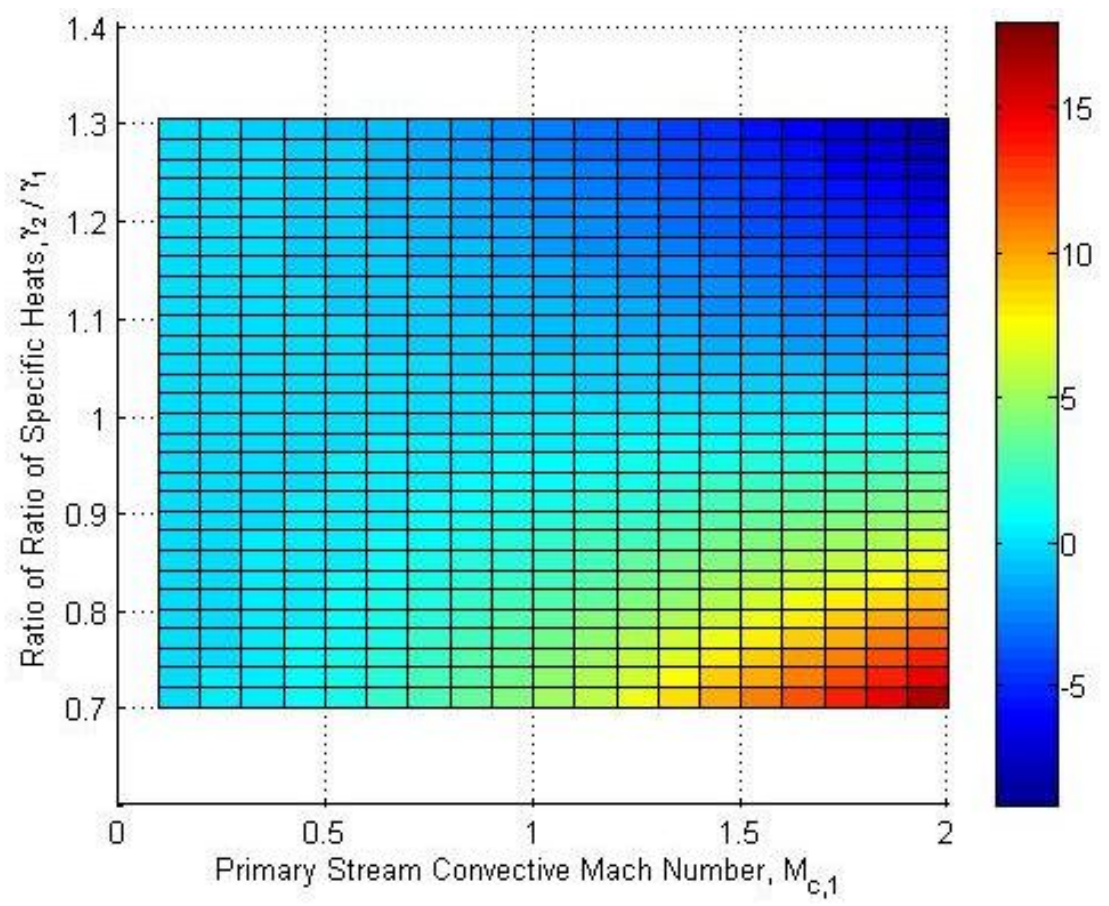

Figure 20. Percent error introduced to $M_{c_{2}}=f\left(M_{c_{1}}\right)$ relationship by the omission of $O\left(M_{c_{1}}^{4}\right)$ terms.

The next step involved calculating the convective velocity, $U_{c}$, in terms of freestream parameters. Once again, the assumption of equal static pressures was called upon. This time, it was used in conjunction with an assumption that both streams are ideal gases. Although the ideal gas assumption is justifiable for a vast variety of cases, the reader should take caution when applying it to hypersonic or high temperature cases such as scramjet fuel injection or air augmented rocket entrainment. These two assumptions combine to simplify the ratio of acoustic speeds to

$$
\frac{a_{2}}{a_{1}}=\sqrt{\frac{\gamma_{2} R_{2} T_{2}}{\gamma_{1} R_{1} T_{1}}}=\sqrt{\frac{\gamma_{2} \rho_{1}}{\gamma_{1} \rho_{2}}}
$$

Through simple algebra, it can be shown that the combination of Eq. 29, Eq. 30, Eq. 33, and Eq. 37 yields

$$
\frac{U_{c}}{U_{1}}=\frac{1+r \sqrt{s}}{1+\sqrt{s}}
$$


If $M_{c_{2}}$ is incorrect by a factor of $\varepsilon$ (i.e. $M_{c_{2}}=\varepsilon \sqrt{\gamma_{2} / \gamma_{1}} M_{c_{1}}$ ), then the propagation of error updates Eq. 38 to the form,

$$
\frac{U_{c}}{U_{1}}=\frac{\varepsilon+r \sqrt{s}}{\varepsilon+\sqrt{s}}
$$

Moreover, if the ideal gas and static pressure assumptions are found to be incorrect by a factor of $\alpha$ (i.e. $\alpha=\rho_{2} R_{2} T_{2} / \rho_{1} R_{1} T_{1}$ ), then the propagation of error updates Eq. 39 to the form,

$$
\frac{U_{c}}{U_{1}}=\frac{\varepsilon \sqrt{\alpha}+r \sqrt{s}}{\varepsilon \sqrt{\alpha}+\sqrt{s}}
$$

This relationship suggests that the error in $U_{c}$ prediction is greatest in the hypothetical case of a low density, high speed jet mixing with a high density stagnant gas of considerably different $\gamma$. If, for example, $\left|\gamma_{2} / \gamma_{1}\right|$ and the true value of $M_{c_{1}}$ were large enough to produce $\varepsilon=1.2$ while $s=7$, then the isentropic relationship with the above simplification would overpredict $U_{c}$ by approximately $14 \%$. The error is further compounded if $\alpha$ is large. In particular, researchers should take special caution when attempting to use this approach to study mixing layers with pressure gradients.

On the other hand, the errors inherent to the above derivation are quite small if any of the parameters are relaxed. That is, if $\gamma_{2} \approx \gamma_{1}$, the true value of $M_{c_{1}}$ is small, the velocity ratio is moderate to high, or the density ratio is low, then either $\varepsilon$ or its effect on Eq. 39 become quite small (i.e. $< \pm 2 \%$ ). These circumstances apply to a vast majority of the cases presently under consideration. Based on these findings, the convective Mach number and the simplified isentropic relationship for the convective speed are confirmed as an acceptable parameter for the study of most compressible turbulent mixing layers. 
No discussion about the prediction of the convective velocity is complete, however, without some mention of the controversy associated with its measurement. Several authors have presented contradicting evidence about the accuracy of the theoretical predictions. Recently, Thurow et al. (2008) published an assessment of the associated measurement techniques in which they found that flow visualization and inadequately seeded planar Doppler velocimetry (PDV) can provide misleading results. Meanwhile, the result from their fully seeded PDV case was close to the theoretically expected value.

\subsection{Normalization and the Incompressible Growth Rate Model}

The third potential source of variance within the compressibility model is the act of normalizing the observed growth rate by an estimate for what the growth rate would have been without the effects of compressibility. Accurate establishment and application of the incompressible growth rate model is necessary for the normalized growth rate to have meaning. The following subsections discuss these factors.

\subsubsection{Derivation of the Incompressible Growth Rate Model}

The accuracy of the incompressible growth rate model by which the observed growth rates are normalized is paramount to the isolation of the effects of compressibility. The standard form of the model is (Papamoschou \& Roshko 1988)

$$
\delta_{0}^{\prime}=C_{\delta} \frac{(1+r)(1+r \sqrt{s})}{1+\sqrt{s}}=C_{\delta} \lambda_{s}
$$

where $C_{\delta}$ is a relationship of proportionality. Specific values for $C_{\delta}$ have been suggested for each of the three velocity-based thickness definitions by Barone et al. (2006). However, complete derivations of parameter-based equations for the determination of $C_{\delta}$ are not 
found in the literature. As such, they are presented here. The products of these derivations (i.e. Eq. 49, Eq. 50, and Eq. 56) are applied in Section 4.3.1.

\section{Overview}

The velocity profile for incompressible mixing layers can be estimated using the error function profile,

$$
\begin{aligned}
U^{*}= & \frac{U-U_{2}}{U_{1}-U_{2}}=\frac{1}{2}\left[1+\operatorname{erf}\left(\frac{\sigma_{0} y}{\lambda_{s} x}\right)\right] \\
& \operatorname{erf}(z)=\frac{2}{\sqrt{\pi}} \int_{0}^{z} e^{-k^{2}} d k
\end{aligned}
$$

The three velocity-based mixing layer thickness definitions (described in more detail in Section 1.1.1) are listed below:

- Shear Layer Thickness: $\delta_{b}=y_{1}-y_{2}$, where $y_{1}$ and $y_{2}$ are the locations where $U^{*}=0.9$ and 0.1 , respectively.

- Stanford Thickness: $\delta_{S}=y_{1}-y_{2}$, where $y_{1}$ and $y_{2}$ are the locations where $U^{*}=$ $\sqrt{0.9}$ and $\sqrt{0.1}$, respectively.

- Vorticity Thickness: $\delta_{\omega}=\left(U_{1}-U_{2}\right) /(\partial U / \partial y)_{\max }=1 /\left(\partial U^{*} / \partial y\right)_{\max }$.

Because mixing layers are self-similar and also because of the nature of the error function velocity profile, the growth rates of these thicknesses vary as functions of $\sigma_{0}, \lambda_{s}$, and the individual definitions (subscript "def" is replaced by " $b$ ", " $S$ ", or " $\omega$ " accordingly) such that

$$
\delta_{0, \text { def }}^{\prime}=C_{d e f}\left(\sigma_{0}\right) * \lambda_{s}
$$

The derivations of $C_{b}\left(\sigma_{0}\right), C_{S}\left(\sigma_{0}\right)$, and $C_{\omega}\left(\sigma_{0}\right)$ are shown below. 


\section{Derivation of $C_{b}$ and $C_{S}$}

The derivations for $C_{b}$ and $C_{S}$ are similar, so only the derivation for $C_{b}$ is shown here. To begin, it is desired to know the values of $y$ when $U^{*}=0.9$ and 0.1 . For $U^{*}=0.9$,

$$
U^{*}=0.9=\frac{1}{2}\left[1+\operatorname{erf}\left(\frac{\sigma_{0} y_{1}}{\lambda_{s} x}\right)\right]
$$

Eq. 45

Solve for $y_{1}$ using the inverse error function to get

$$
y_{1}=\frac{\lambda_{s} x}{\sigma_{0}} \operatorname{erf}^{-1}[(0.9 * 2)-1]
$$

Repeat the process for $U^{*}=0.1$ and take the difference to yield the shear layer thickness,

$$
\delta_{b}=y_{1}-y_{2}=\frac{\lambda_{s} x}{\sigma_{0}}\left[\operatorname{erf}^{-1}(0.8)-\operatorname{erf}^{-1}(-0.8)\right] \cong 1.8124 \frac{\lambda_{s} x}{\sigma_{0}}
$$

Take the derivative with respect to $x$ to yield the mixing layer growth rate,

$$
\delta_{b}^{\prime} \cong 1.8124 \frac{\lambda_{s}}{\sigma_{0}}
$$

Finally, rearrange terms into the coefficient format,

$$
C_{b}=\frac{\delta_{b}^{\prime}}{\lambda_{s}} \cong \frac{1.8124}{\sigma_{0}}
$$

Through nearly equivalent steps, the Stanford thickness can be found to be

$$
C_{S} \cong \frac{1.4923}{\sigma_{0}}
$$

\section{Derivation of $C_{\omega}$}

For the derivation of the incompressible vorticity thickness growth rate coefficient, note that the derivative of the error function is as follows:

$$
\frac{d}{d z} \operatorname{erf}(z)=\frac{2}{\sqrt{\pi}} e^{-z^{2}}
$$

Begin by calculating the $y$-derivative of the error function velocity profile, 


$$
\frac{\partial U^{*}}{\partial y}=\frac{\partial}{\partial y}\left[\frac{1}{2}\left[1+\operatorname{erf}\left(\frac{\sigma_{0} y}{\lambda_{s} x}\right)\right]\right]=\frac{\sigma_{0}}{\sqrt{\pi} \lambda_{s} x} e^{-\left(\frac{\sigma_{0} y}{\lambda_{s} x}\right)^{2}}
$$

This derivative is at its maximum when $y=0$. Therefore,

$$
\left(\frac{\partial U^{*}}{\partial y}\right)_{\max }=\frac{\sigma_{0}}{\sqrt{\pi} \lambda_{s} x}
$$

The vorticity thickness is the inverse of this derivative,

$$
\delta_{\omega}=\frac{1}{\left(\frac{\partial U^{*}}{\partial y}\right)_{\max }}=\frac{\sqrt{\pi} \lambda_{s} x}{\sigma_{0}}
$$

Take the derivative with respect to $x$ to yield the growth rate,

$$
\delta_{\omega}^{\prime}=\frac{\sqrt{\pi} \lambda_{s}}{\sigma_{0}}
$$

Finally, rearrange terms into the coefficient format,

$$
C_{\omega}=\frac{\delta_{\omega}^{\prime}}{\lambda_{s}}=\frac{\sqrt{\pi}}{\sigma_{0}}
$$

\subsubsection{Apparent Dependence on Mixing Layer Thickness Definition}

The normalized growth rate, $\phi$, is created by dividing an observed growth rate with an incompressible growth rate model evaluated with the same $r, s$, and thickness definition as the observed case. The fact that the thickness definition can affect the incompressible growth rate estimate implies that the resulting normalized values of $\phi$ should be independent of the chosen thickness definition.

To test this notion, the unaltered database from Figure 14 was separated into categories based on the thickness definition of the data as shown in Figure 21. The velocity-based thicknesses category contains all data that was reported in terms of $\delta_{b}, \delta_{S}$, or $\delta_{\omega}$. The visual thicknesses category contains only the data that was extracted from flow imaging 
and reported as $\delta_{v i s}$. The density-based thicknesses category contains any thickness definition that is directly affected by the density profile, including $\delta_{\theta}, \delta_{p i t}$, and $\delta_{\rho m}$.

Visual inspection of the $\phi$ vs. $M_{c}$ plot in Figure 21 and the associated histogram of residuals in Figure 22 suggests that a discrepancy may exist between the velocity-based thicknesses and the other types. Moreover, it appears that the Dimotakis (1991a) trendline represents the visual thickness growth rates much better than the velocity-based thickness growth rates. Such a discrepancy could indicate that the density profile and the velocity profile may be affected differently by compressibility. Another possible explanation could be that the incompressible growth rate models for the velocity-based thicknesses and the density-based or visual thicknesses are not consistent with each other. Either way, the evidence shown here suggests that (with the present knowledge) a trendline for the velocity-based thickness growth rates should be fit without regard for the visual and density-based thicknesses. The data processing and model fitting approach in Section 4 reflects this decision. 


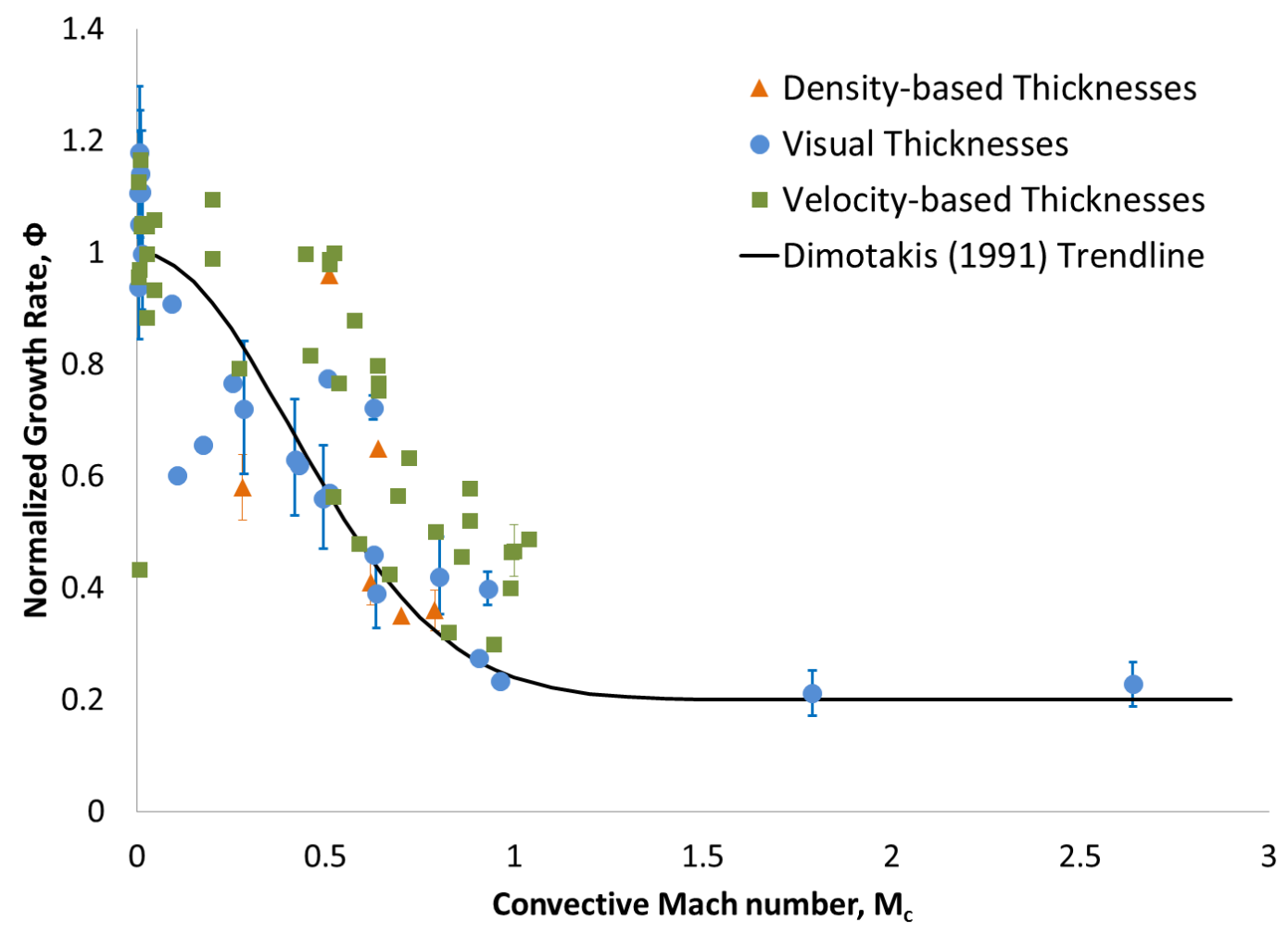

Figure 21. The unaltered mixing layer database represented in terms of convective Mach number and identified by thickness definition type.

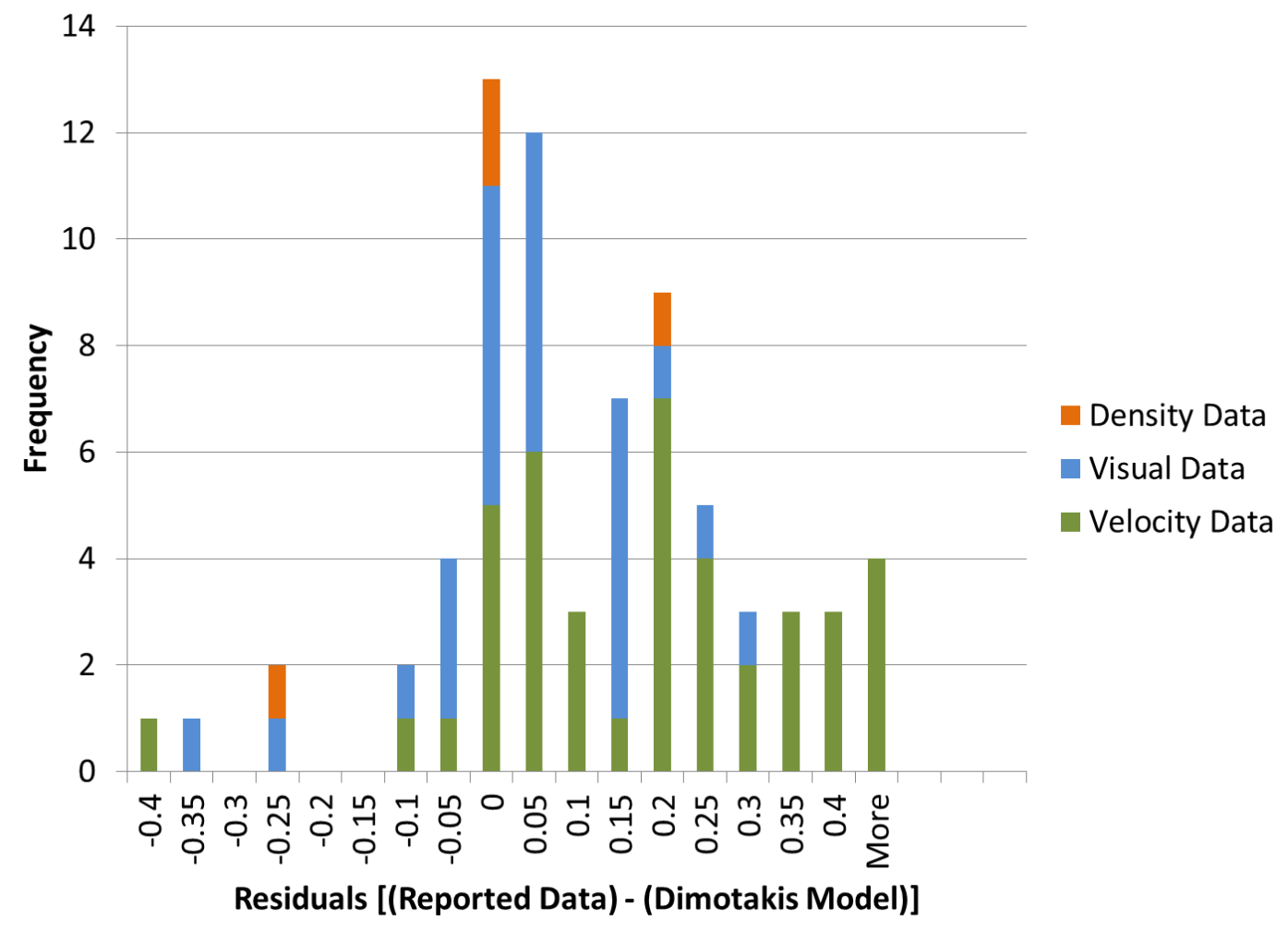

Figure 22. Histogram of residuals for Figure 21. 


\subsubsection{Inconsistent Normalization Case Example: Data from Chinzei et al.}

(1986)

What may be the most significant contributor for the large scatter in the data is the lack of consistency seen in authors' representations of their predecessors' work. It is customary to present comparable data from past studies alongside the results of one's own publication. The collection and treatment of each individual set of data is subject to many sources of augmentation that are capable of effectively altering the data's reception. As the original publications become older and less accessible, authors may be inclined to extract their data sets from secondary sources, which can compound the possibility of changing how the original data is represented.

Take the experimental data published by Chinzei et al. (1986) as a case example. Since it was published before the theoretical work of Papamoschou and Roshko (1988), the measured mixing layer growth rates were normalized according to a process that is quite different from the presently accepted incompressible growth rate models. As a result, each author wanting to compare his/her own results with those of Chinzei et al. had to make a decision about how to properly normalize the growth rates. In contrast, most of the experiments that came after 1988 were normalized by modern incompressible growth rate estimates within the original publication, thereby making it easier for future authors to reproduce the exact normalized growth rate without having to concern themselves with the raw, experimentally measured growth rate.

The outcome of the added freedom associated with the data from Chinzei et al. (1986) is shown in Figure 23. Each data series in this plot supposedly represents the exact same 
outcome of one experiment, however they were each extracted from a different source. The variables between these data series are the models used for the incompressible growth rate and each author's ability to accurately reproduce the original data. It is clear that the incompressible growth rate model used for normalization can play a significant role in how a raw data set is perceived. Moreover, the model appears to have taken many forms over the years. These variations suggest that a major source of the disagreement within the overall database could have been caused by inconsistent post-processing rather than from the experiments themselves.

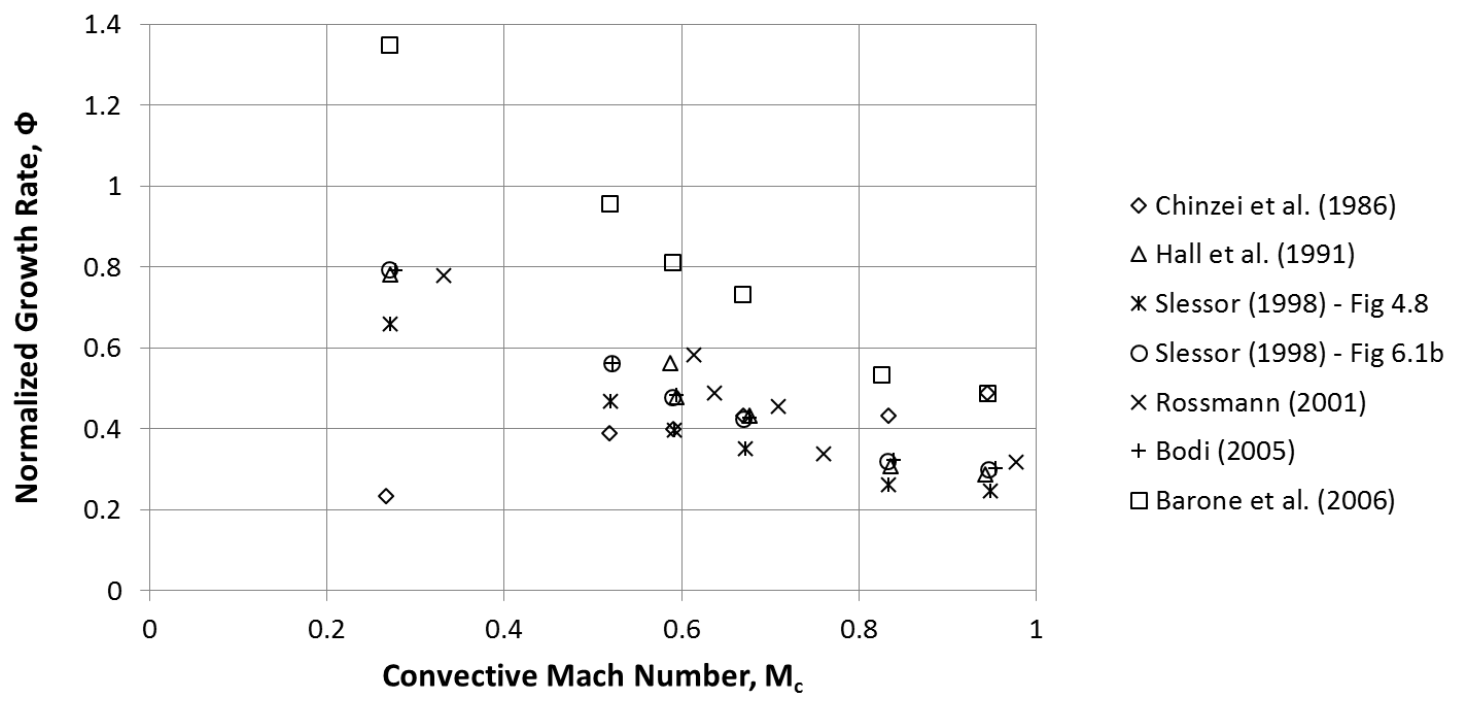

Figure 23. Various representations of the growth rates observed by Chinzei et al. (1986).

The compilation of data in the present study addresses the inconsistencies shown above in two ways. First, all of the data was extracted from their original publications in order to avoid copying erroneously translated information. Secondly, enough information was required of each case in order to clearly identify the observed mixing layer growth rate. A selection of article summaries and comments on data extraction is included in Appendix $D$ to clarify the processes used in some of the more difficult cases. The benefit derived 
from the careful data extraction process employed here was maintained in the modeling approach described in Section 4 by consistently applying a re-tuned incompressible growth rate model to all of the applicable data.

\subsection{Dimotakis (1991a) Trendline, Revisited}

The final aspect in creating a useful and trustworthy empirical model is the parametric function that is fitted to the data. The function used here as the basis of the model was generalized from the trendline originally suggested by Dimotakis (1991a). The function has the form

$$
\widehat{\phi}=(1-A) e^{-B\left(M_{C}\right)^{C}}+A
$$

where $A, B$, and $C$ are the parameters of the model. As presented by Dimotakis (1991a), these parameters had values of $A=0.2, B=3$, and $C=2$.

Since this function is the basis of the fitted model, further analysis of its form and sensitivities is warranted. The requirements of the function, as defined by conventionally accepted knowledge about compressible turbulent mixing layers and the data set in question, are listed below.

1. The model must have $\hat{\phi}=1$ at $M_{c}=0$.

2. Until some low value of $M_{c}$ (typically around $M_{c} \sim 0.3$ ), $\hat{\phi} \approx 1$.

3. For $M_{c} \gtrsim 1, \hat{\phi}$ is constant. The exact $M_{c}$ location and $\hat{\phi}$ constant value are allowed to vary with the data.

4. The curve should have a smooth transition between the "beginning" (requirement \#1) and the "end" (requirement \#3). 
Figure 24 through Figure 26 show how the model created by Dimotakis and expanded here meets these requirements while allowing sufficient flexibility to the statistician. In each figure, a single parameter is varied while the other parameters are fixed at nominal (approximate) values. In each, a bold curve indicates where the varying parameter equals the nominal value used in the other figures. The values of the parameters being plotted are listed above each figure.

Figure 24 shows how parameter $A$ sufficiently enables the model to meet requirements \#1 and \#3 while providing simple and precise control of the asymptotic value. According to the requirements, appropriate values of $A$ are within the set $(0,1)$ depending on the magnitude of compressibility's effect.

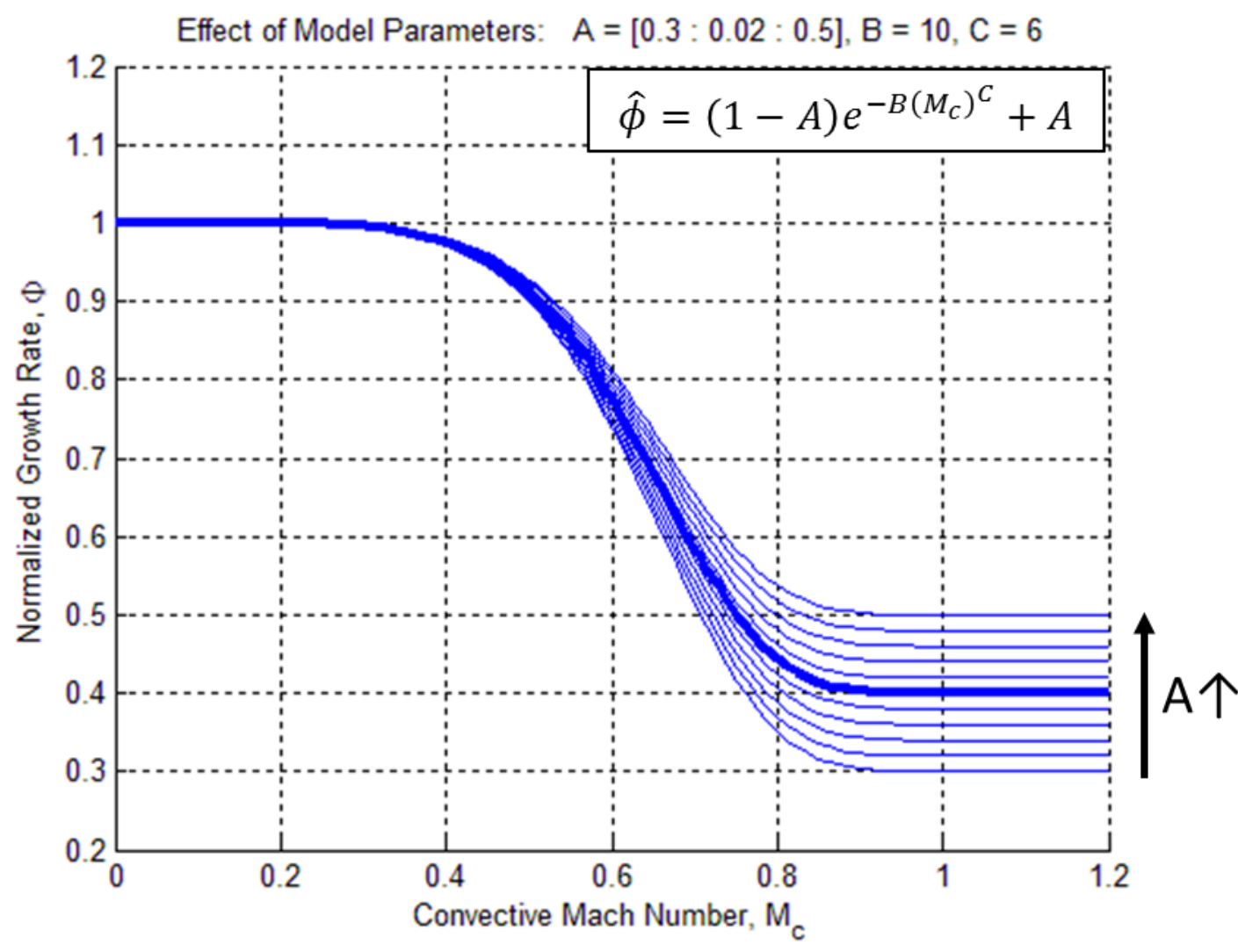

Figure 24. The effect of model parameter "A". 
Figure 25 shows how parameter $B$ can be used to tune the value of $M_{c}$ at which the model reaches its asymptotic $\hat{\phi}$ value. According to the requirements, appropriate values of $B$ are within the set $(0, \infty)$.

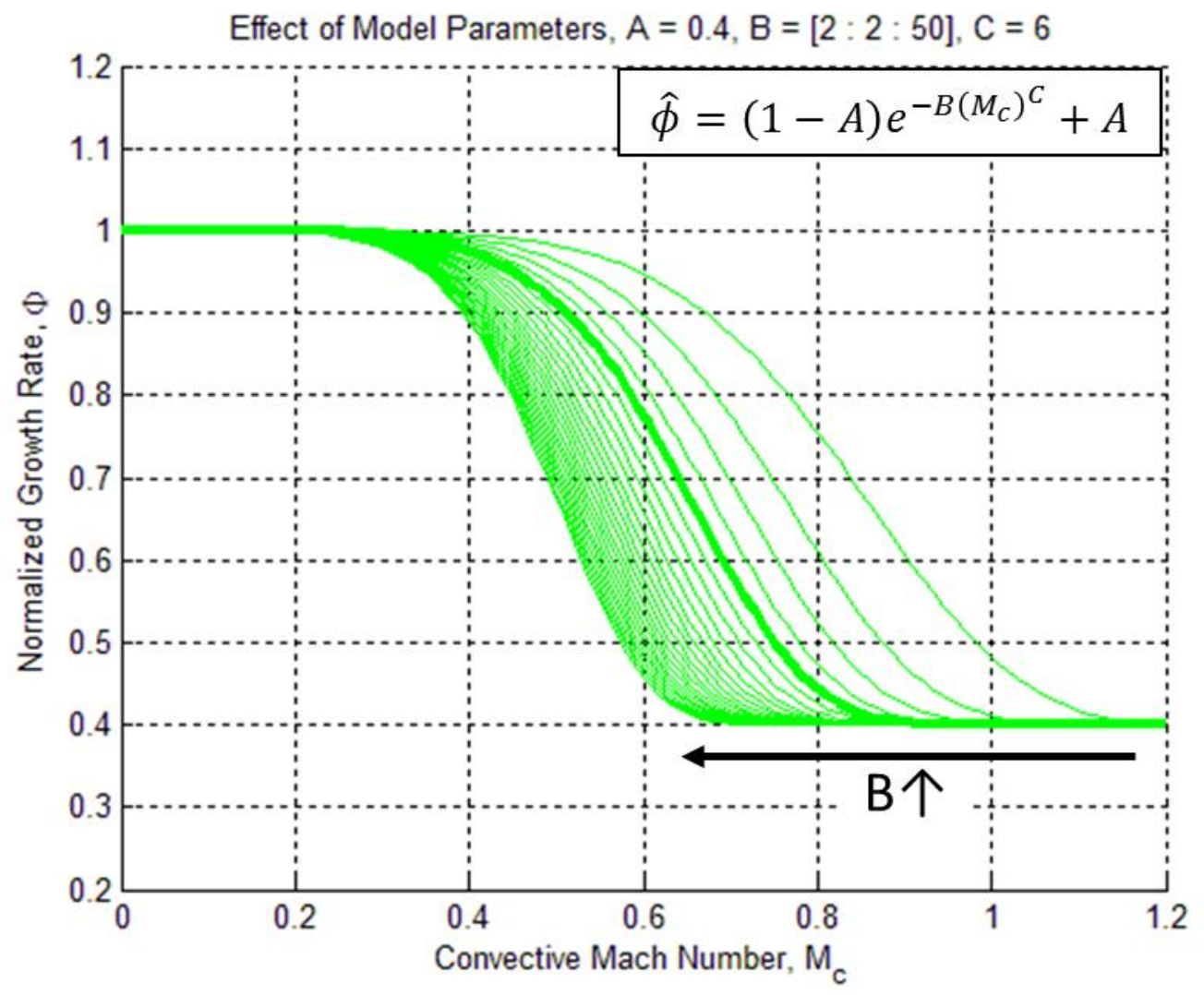

Figure 25. The effect of model parameter "B".

Figure 26 shows how parameter $C$ can be used to tune the value of $M_{c}$ at which the model departs from the incompressible $(\hat{\phi} \approx 1)$ region. According to the requirements, appropriate values of $C$ are approximately within the set $(2,40)$. Although these suggested limits can be crossed if desired, the smoothness of the curve is largely impacted. 


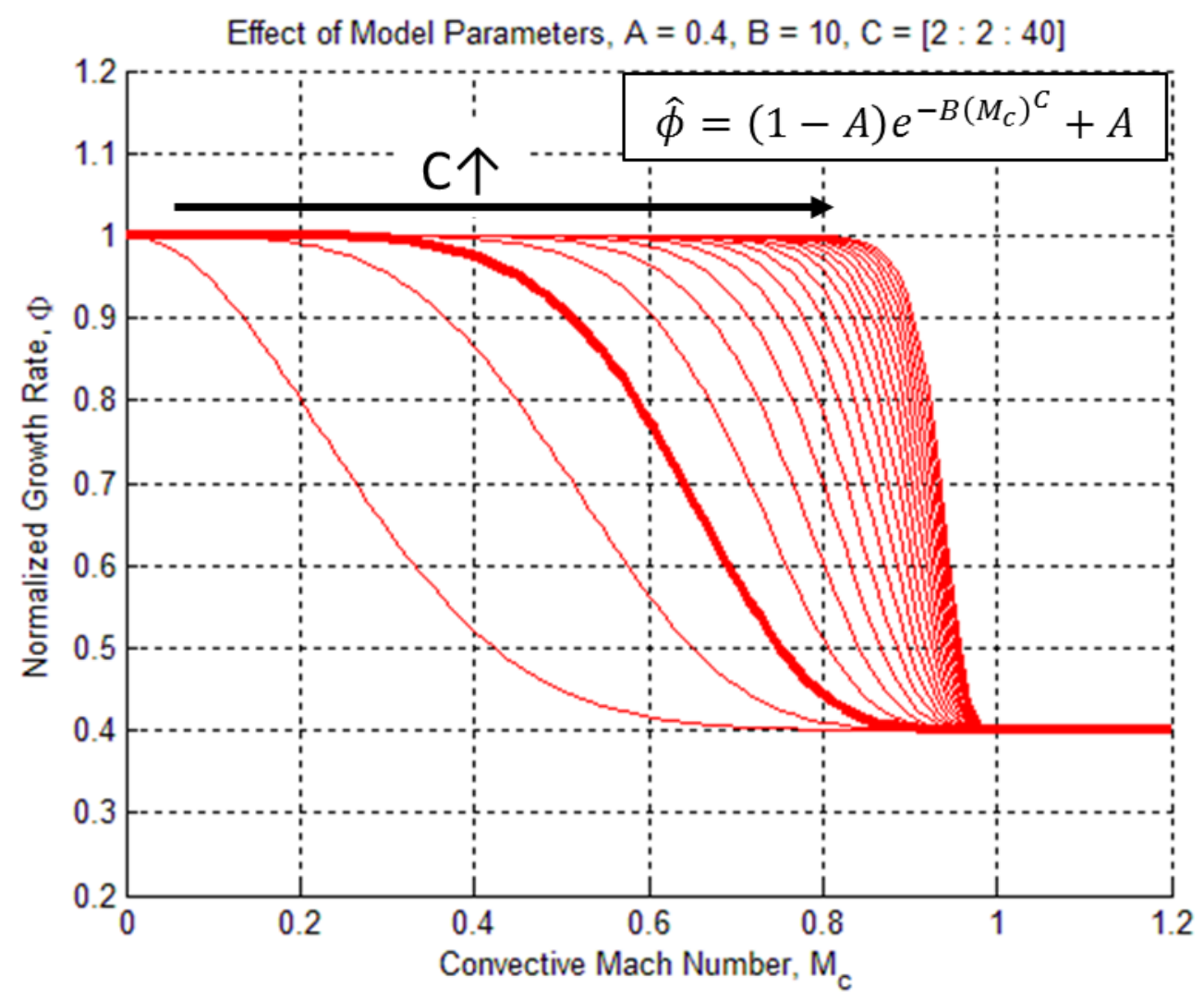

Figure 26. The effect of model parameter " $\mathrm{C}$ ".

It should be noted that the parameters $B$ and $C$ are not mutually exclusive. Both parameters affect the transition between the incompressible and highly compressible asymptotes in a similar fashion—higher values lead to sharper transitions, and vice versa. If the two parameters are not relatively well balanced, the larger parameter can dominate over the smaller one, affecting both the incompressible and highly compressible regions. This coupled relationship makes appropriate selection of parameter values very difficult without the aid of numerical optimizers.

Although parameter selection may be somewhat difficult, the form of the model adequately meets the minimal requirements. Moreover, the features of the model are sufficiently sensitive to the model's parameters. 


\section{A New Model for Turbulent Mixing Layer Growth Rates}

The analysis presented in Section 3 was used as a foundation for an improved model for the turbulent mixing layer growth rate. Sections 3.1 and 3.2 suggest that the convective Mach number is an adequate measure of compressibility. Meanwhile, Section 3.3 identifies the incompressible growth rate model as a prime candidate for modification. Based on the findings of Section 3.3.2 and the availability of parametric equations for velocity-based incompressible growth rates, the model is fit to only the velocity-based thickness data. Finally, Section 3.4 shows that a generalized, parametric form of the Dimotakis (1991a) model function is suitable for representing the trends found in the data.

In preparation for the modeling process, Section 4.1 identifies the statistical tools that are employed, and Section 4.2 presents the reduced data set that is considered. The process itself is quite simple, with proper tuning and consistent application of the incompressible growth rate model in Section 4.3 and the identification and removal of statistical outliers in Section 4.4 .

\subsection{Discussion of Statistical Tools Used During the Modeling Process}

This section offers a brief overview of the statistical tools that are used during the modeling process. Among these tools are least square best-fit model parameter tuning, the coefficient of determination, histograms of residuals, and boxplots. 


\section{Model Parameter Tuning}

The model's parameters were tuned to fit the data for the least squared sum of residuals. The incompressible growth rate model has only one parameter, $\sigma_{0}$, which was tuned using an optimization algorithm written in MATLAB (see Section 4.3). The model for the normalized growth rate has three parameters, $A, B$, and $C$, which were tuned using a program called ZunZun. ZunZun is an open-source curve fitting tool that was written by James R. Phillips, is powered by Python, and is available on the internet at http://zunzun.com/. It runs a very robust regression algorithm that is capable of finding best-fit parameter sets for hundreds of two-dimensional and three-dimensional nonlinear equations in addition to user-defined functions. The user-defined function feature was used in the present effort. A plethora of statistical metrics and plots are reported for each curve fit so that the user can assess the quality of the model.

\section{Goodness-of-Fit Metrics}

The coefficient of determination $\left(R^{2}\right)$ can be used to describe how well a model fits the data. More specifically, $R^{2}$ is a measure of how well the variance in the data is described by the model. It is defined as

$$
R^{2}=1-\frac{\sum_{i}\left(p_{i}-f_{i}\right)^{2}}{\sum_{i}\left(p_{i}-\bar{p}\right)^{2}}
$$

where $p_{i}$ is the set of observed data, $\bar{p}$ is the arithmetic mean of the observed data, and $f_{i}$ is the output of the model for the same independent variables associated with the respective $p_{i}$. There are criticisms about $R^{2}$ because it is only an index of proximity between the data points and the curve and not an index of the correctness of the model (Christopoulos \& Lew 2000). 
As a minimum, the use of $R^{2}$ should be paired with an analysis of the residuals to ensure that the correct model is chosen. Graphical representation of the residuals as histograms and scatter plots is often an adequate approach for finding any systematic deviations of the data from the model, which would indicate that the model is incorrect. Ideally, the residuals would be normally distributed (i.e. histogram of residuals resembles a bell curve) and small in magnitude.

\section{Outlier Identification}

Further analysis of the residuals is necessary to identify the possible existence of outliers. The use of boxplots for this purpose is simple yet effective, although it does have some limitations (Hodge \& Austin 2004). In boxplots, the median, lower quartile, and upper quartile are plotted to form a box as shown in Figure 27. Then, the inner quartile range is calculated as the difference between the upper and lower quartiles. The "extremes" reach to the most extreme data points that are within 1.5 times the inner quartile range of the upper and lower quartiles. Outliers are any points that lie beyond the extremes.

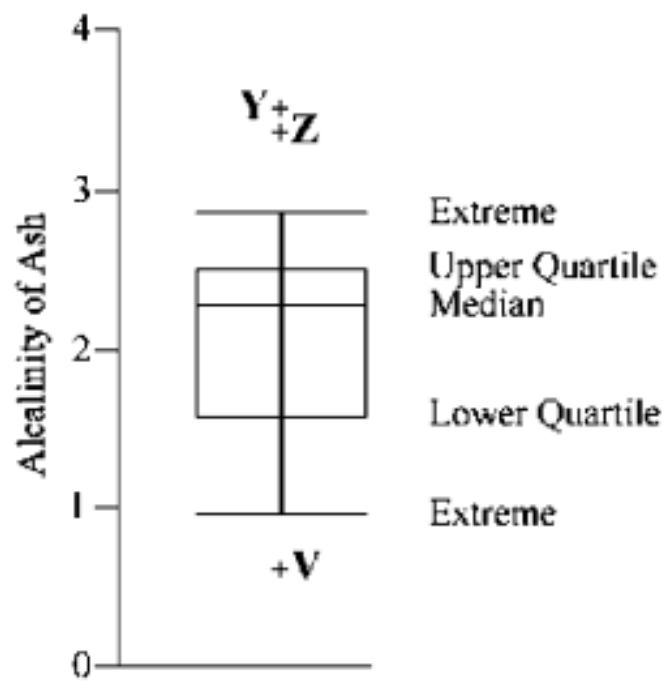

Figure 27. A sample boxplot, from Hodge and Austin (2004). 
In the present case of fitting data to a nonlinear regression, outliers are identified by creating a boxplot of the residuals between the data set and the regression. Any outliers that are identified are removed from the data set, and then a new boxplot is made to reflect the change. This process is repeated until no outliers are found. Ideally, the final boxplot will have no outliers, the median will be located where the residual is zero, and the lower and upper quartiles and extremes will display symmetry about the median. The approach used here is among the simplest of outlier identification techniques and can easily be applied by researchers without a strong background in statistics.

\subsection{Baseline Data Set}

The data fitting began with the velocity-based subset of the complete database presented in Section 2.5. This reduced data set, named Data Set \#0, serves as the baseline for later modifications. It includes 41 velocity-based mixing layer thickness growth rate observations from 11 sources. The model parameters discussed in Section 3.4 were reevaluated using ZunZun to provide an appropriate basis of comparison for the future steps. For Data Set \#0, the best-fit model parameters are $A=0.4428, B=7.9983$, and $C=4.8881$. Data Set \#0 and its best-fit regression are shown in Figure 28. For the sake of clarity, refer to Appendix $C$ to find a table with the data points and their respective citations (Data Set \#0 can be derived from the complete, unaltered database by sorting out the shear layer, Stanford, and vorticity thicknesses). 


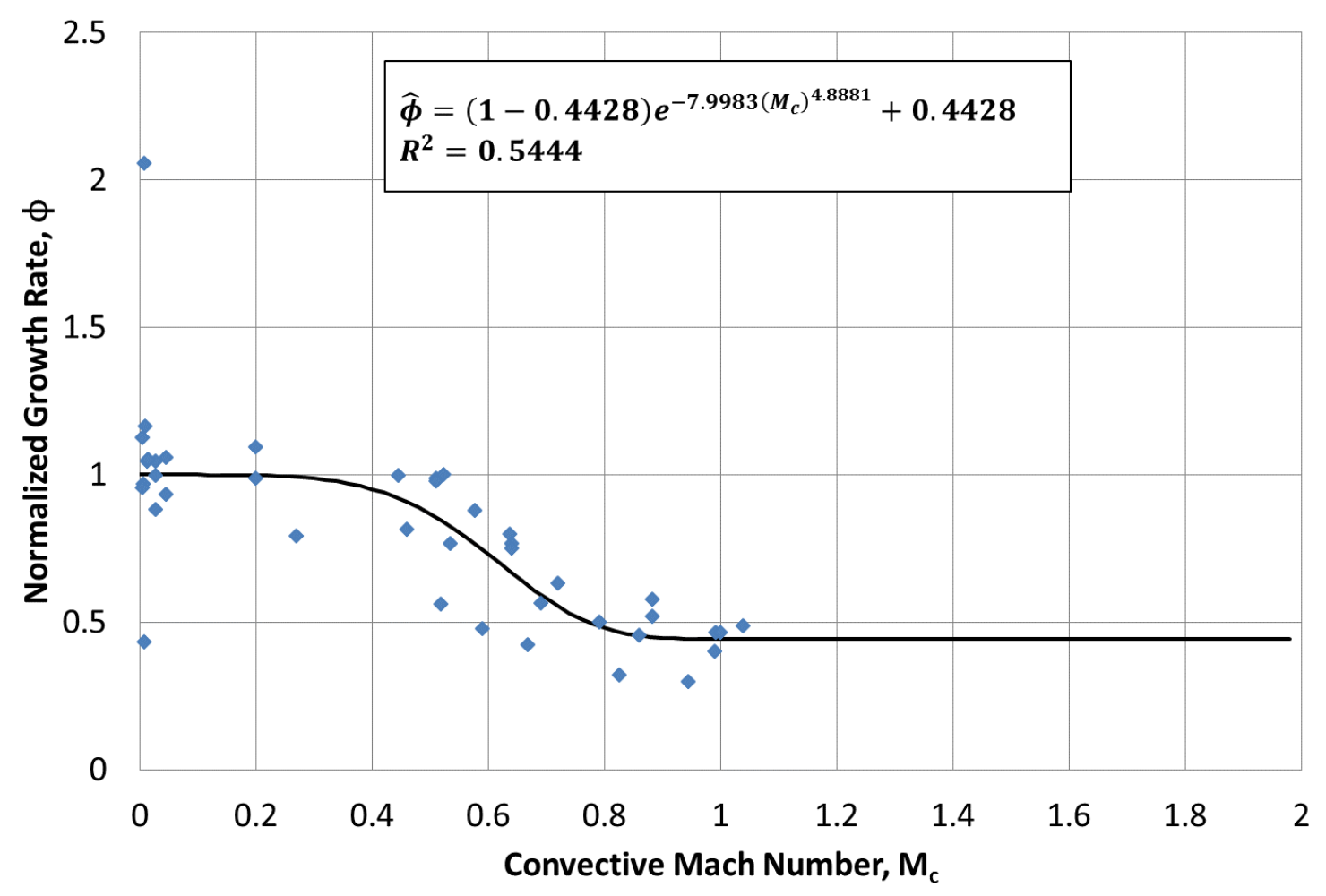

Figure 28. Data Set \#0 - Unmodified velocity-based data set with regression.

The first things one should notice are the two obviously errant data points in the incompressible region. These points are addressed in Section 4.3.1. These two points are largely responsible for the very low value of $R^{2}=0.5444$; if they were omitted, the same regression would yield $R^{2}=0.7974$, which is considerably better but still not sufficient for accurate prediction.

Figure 29 shows a histogram of residuals (observed value minus model value) for Data Set \#0. At first glance, the data appear to be shaped as a bell curve. However, one must recognize that there is a distinct positive shift in the trend. 


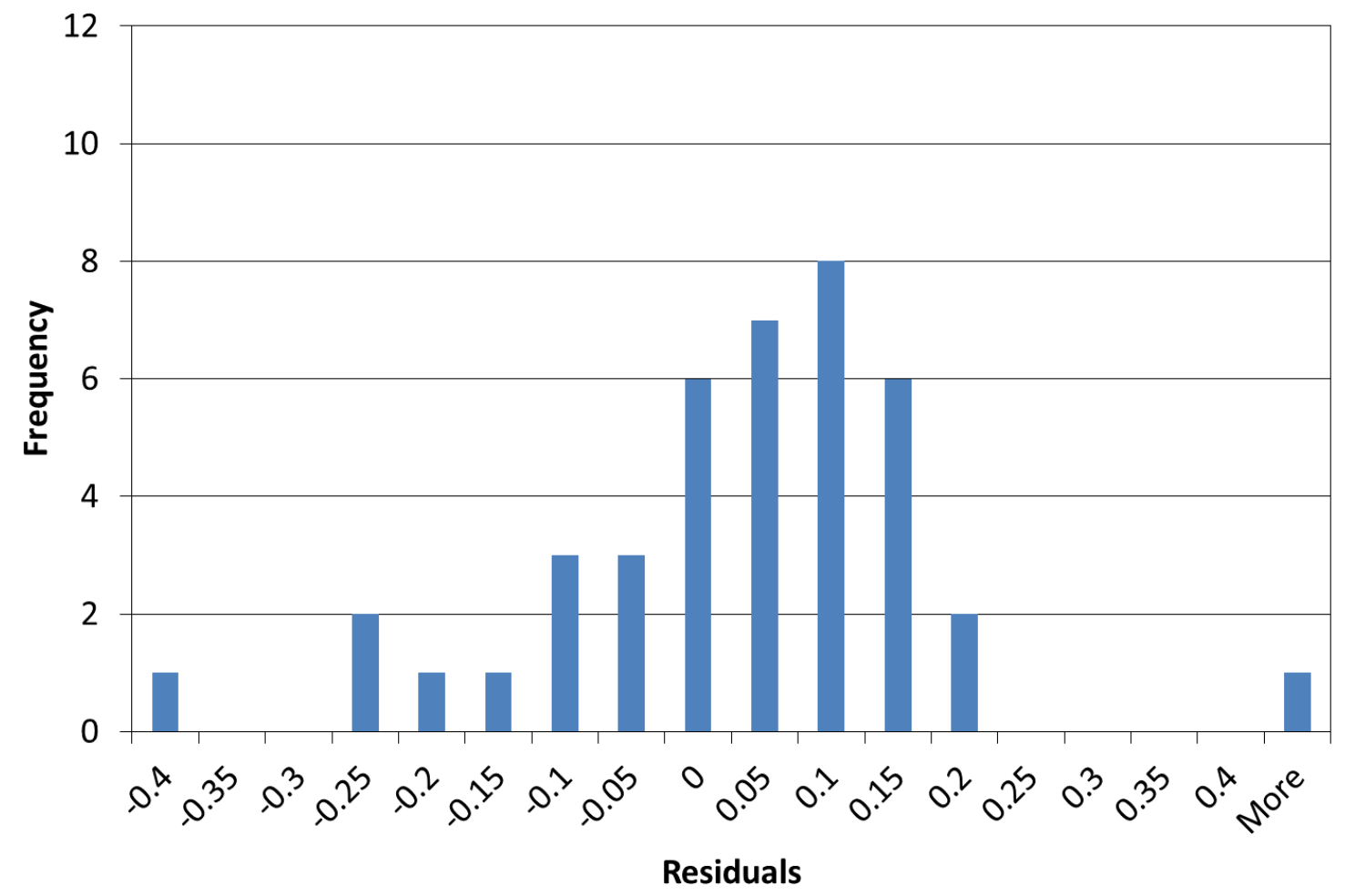

Figure 29. Data Set \#0 - Histogram of residuals.

\subsection{Tuning the Incompressible Growth Rate Model}

As suggested by the discussion in Section 3.3, the first step in improving the data set and the best-fit model associated with it is to re-evaluate the incompressible growth rate model that is used to normalize the observed growth rates. The incompressible model must then be consistently applied to the entire data set.

\subsubsection{Application of Coefficient Functions for Incompressible Growth Rate Model Tuning}

Modern computing capability provides a relatively simple yet accurate approach to the tuning of the empirical constant, $\sigma_{0}$. The approach used here applies MATLAB's fminbnd function, a robust bounded function minimizer, to determine the optimal value of $\sigma_{0}$ 
(however most optimizer algorithms would suffice). The algorithm varied $\sigma_{0}$, and the function in question determined the sum of squared residuals between a fixed set of incompressible observed growth rate data and the growth rate model which was defined by $\sigma_{0}$. The incompressible data set included all of the viable points from Data Set \#0 for which $M_{c} \leq 0.3$. The limit of 0.3 was chosen from visual inspection of the data which suggests that the effect of compressibility becomes significant beyond that value, which agrees with compressibility analysis of single stream flows. The incompressible data set is shown in Table 1. The incompressible growth rate model (see Section 3.3.1) was evaluated for each datum at each iteration of $\sigma_{0}$.

Table 1. Incompressible mixing layer data set.

\begin{tabular}{llllll} 
Author & Year & Thickness Type & $\mathrm{M}_{\mathrm{c}}$ & $\lambda_{\mathrm{s}}$ & $\delta^{\prime}{ }_{\mathrm{obs}}$ \\
\hline Liepmann \& Laufer & 1947 & Shear Layer & 0.026 & 1.000 & 0.164 \\
Liepmann \& Laufer & 1947 & Stanford & 0.026 & 1.000 & 0.120 \\
Liepmann \& Laufer & 1947 & Vorticity & 0.026 & 1.000 & 0.169 \\
Birch \& Eggers (Case 4 - Lee) & 1973 & Shear Layer & 0.046 & 0.481 & 0.084 \\
Birch \& Eggers (Case 4 - Lee) & 1973 & Stanford & 0.046 & 0.481 & 0.061 \\
Brown \& Roshko & 1974 & Vorticity & 0.004 & 0.560 & 0.107 \\
Brown \& Roshko & 1974 & Vorticity & 0.005 & 0.372 & 0.060 \\
Brown \& Roshko & 1974 & Vorticity & 0.006 & 1.133 & 0.187 \\
Brown \& Roshko & 1974 & Vorticity & 0.007 & 1.823 & 0.134 \\
Brown \& Roshko & 1974 & Vorticity & 0.008 & 0.689 & 0.241 \\
Brown \& Roshko & 1974 & Vorticity & 0.009 & 0.449 & 0.089 \\
Brown \& Roshko & 1974 & Vorticity & 0.012 & 0.755 & 0.134 \\
Brown \& Roshko & 1974 & Vorticity & 0.014 & 1.009 & 0.180 \\
Chinzei et al. & 1986 & Stanford & 0.270 & 0.147 & 0.027 \\
Messersmith et al. & 1990 & Shear Layer & 0.200 & 0.116 & 0.021 \\
Goebel \& Dutton & 1991 & Shear Layer & 0.200 & 0.123 & 0.020
\end{tabular}

The optimization algorithm described above was completed multiple times to identify any existing outliers, which were eliminated after each repetition. The iteration was continued 
until no outliers were present to ensure that the remaining data set was not contaminated by outliers.

Figure 30 shows a boxplot of the residuals after the first pass of the iteration. In the case shown here, two outliers are present (Note: these were the obviously errant data points identified in Section 4.2). Analysis of the function outputs identified the two outliers as the $\lambda_{s}=1.8229$ and $\lambda_{s}=0.6890$ cases from Brown and Roshko (1974), who noted large scatter for their cases with $U_{2}=0$, of which these are two. Brown and Roshko suggested that the $U_{2}=0$ cases could have caused difficulties in measurement due to increased sensitivities within the low-speed environment.

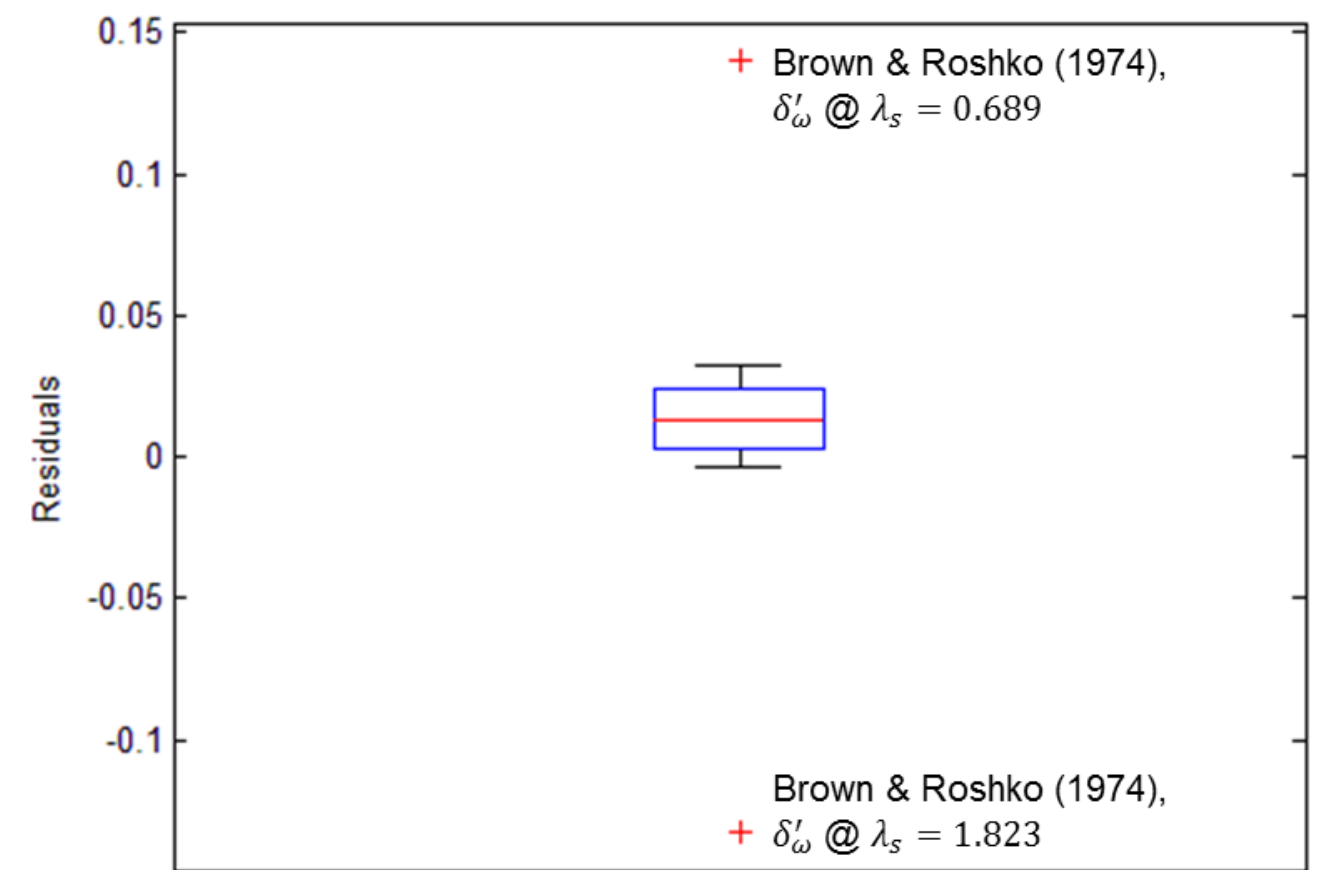

Figure 30. Boxplot of residuals for the first pass of incompressible growth rate model tuning.

After eliminating the two outliers from Figure 30, the optimization algorithm was repeated. As shown in Figure 31, the initial refinement provided the opportunity for a new outlier to be identified. This new outlier was the Stanford thickness measured from the experimental 
case by Liepmann and Laufer (1947). This reference was one of the few for which three different thickness definitions could be applied to a single test case. While it is curious that only one of the three observations would be identified as an outlier, it does not necessarily imply that the other data associated with Liepmann and Laufer's test case are invalid.

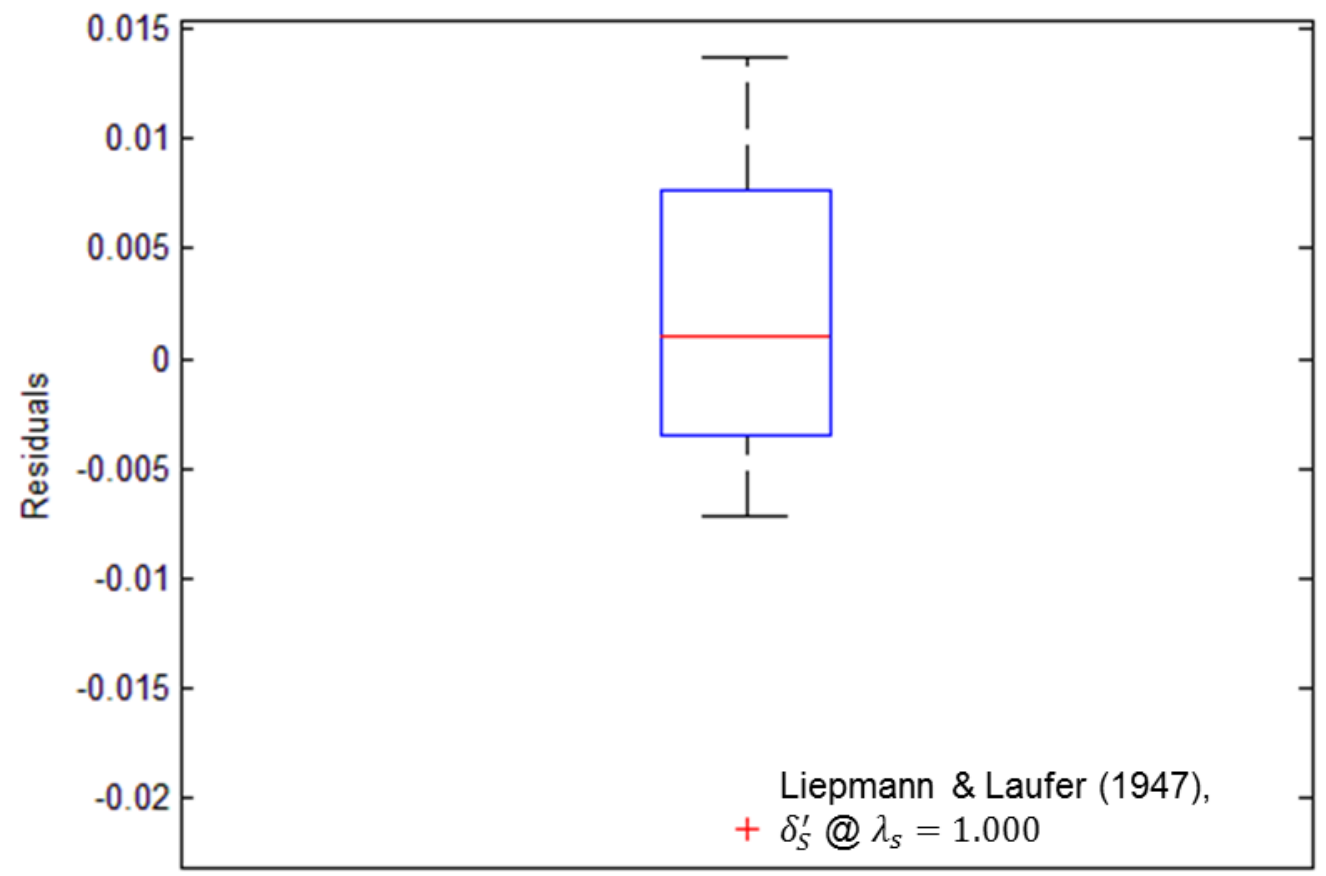

Figure 31. Boxplot of residuals for the second pass of incompressible growth rate model tuning.

After the elimination of the outlier identified in the second pass, a third and final optimization was performed. Figure 32 shows that no new outliers were identified and that the median of the residuals (denoted by the red line) is very nearly at 0 . Furthermore, the defining features of the boxplot are all fairly well centered about the median. 


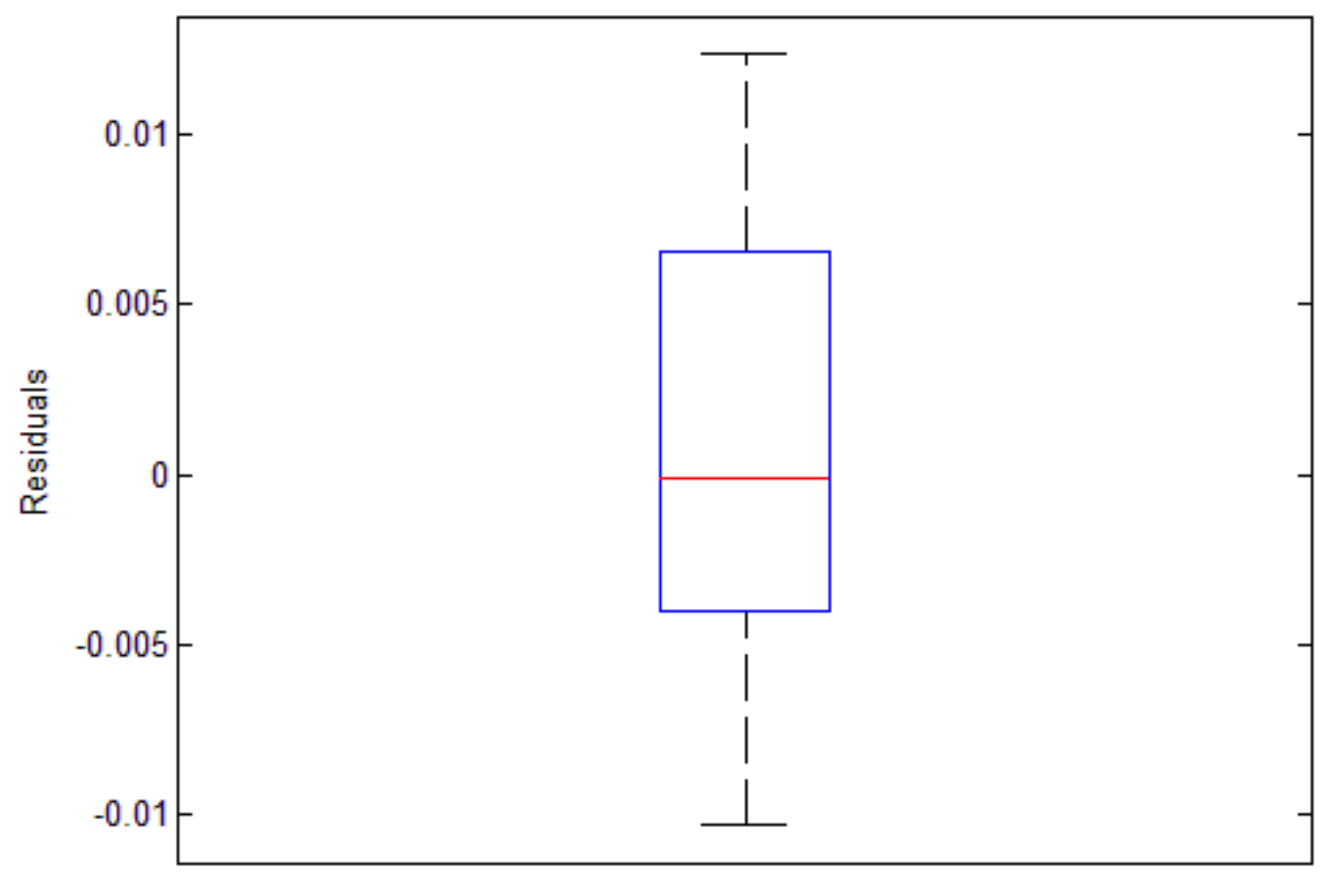

Figure 32. Boxplot of residuals for the third pass of incompressible growth rate model tuning.

The result of the process was a value for $\sigma_{0}$ that most closely fits the available aggregate of incompressible growth rate data without giving preference to any one experiment. This value is $\sigma_{0}=10.3773$, which is close yet notably less than the conventionally accepted value of $\sigma_{0}=11.0$. Table 2 shows the difference in model coefficients for the different thickness definitions for the conventional and new values of $\sigma_{0}$.

Table 2. Comparison of incompressible model coefficients for $\sigma_{0}=11.0$ and $\sigma_{0}=$ 10.3773.

\begin{tabular}{|c|c|c|}
\hline Coefficient & $\begin{array}{c}\text { Error Function Model } \\
\mathbf{\sigma}_{0}=11.0\end{array}$ & $\begin{array}{c}\text { Error Function Model } \\
\mathbf{\sigma}_{0}=10.3773\end{array}$ \\
\hline $\mathrm{C}_{\mathrm{b}}$ & 0.1648 & 0.1746 \\
\hline $\mathrm{C}_{\mathrm{s}}$ & 0.1357 & 0.1438 \\
\hline $\mathrm{C}_{\omega}$ & 0.1611 & 0.1708 \\
\hline
\end{tabular}


The resulting coefficient of determination for this tuned model, $R^{2}=0.9856$, is a marked improvement over the value of $R^{2}=0.5603$ found when using $\sigma_{0}=11.0$ for the entire incompressible data set listed in Table 1. It is also a slight improvement over the value of $R^{2}=0.9731$, which was found for $\sigma_{0}=11.0$ but with the outliers removed.

Figure 33 shows a histogram of residuals as another diagnostic plot for the incompressible model. Ideally, a histogram of residuals would have a normal distribution. The present model is afflicted by an underwhelming amount of data points, and as such, does not display the iconic bell curve that is desired. In spite of the unfortunate scarcity, certain features of the plot which suggest normalcy can be identified. For instance, the highest frequency is found very close to zero. Furthermore, all of the residuals seem moderately well centered about zero.

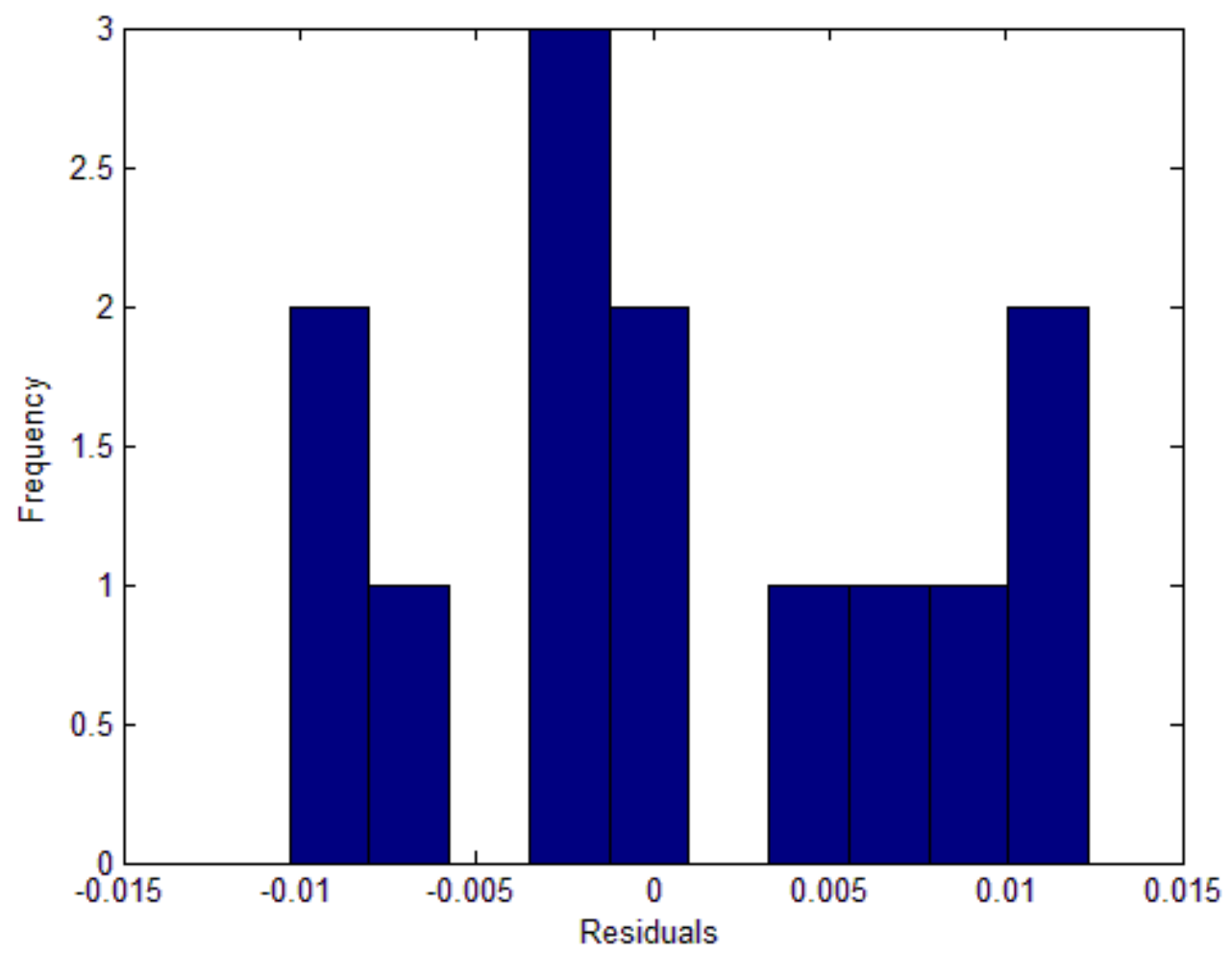

Figure 33. Histogram of residuals for the incompressible growth rate model. 


\subsubsection{Updated Data Set with Consistently Applied Incompressible Growth Rate Model}

The coefficients from Table 2 were used to predict incompressible mixing layer growth rates (according to Eq. 41, page 46) for all of the cases in Data Set \#0. These incompressible growth rates then replaced the existing values that the authors originally predicted in their publications. The outliers that were identified from the incompressible data set in Section 4.3.1 were removed from the Data Set \#0. The result of these modifications, called Data Set \#1, was processed in ZunZun to produce a new best-fit model, as shown in Figure 34. For Data Set \#1, the best-fit model parameters are $A=$ 0.4636, $B=17.0677$, and $C=7.4541$. As before, refer to Appendix $C$ to find a table with the data points and their respective citations.

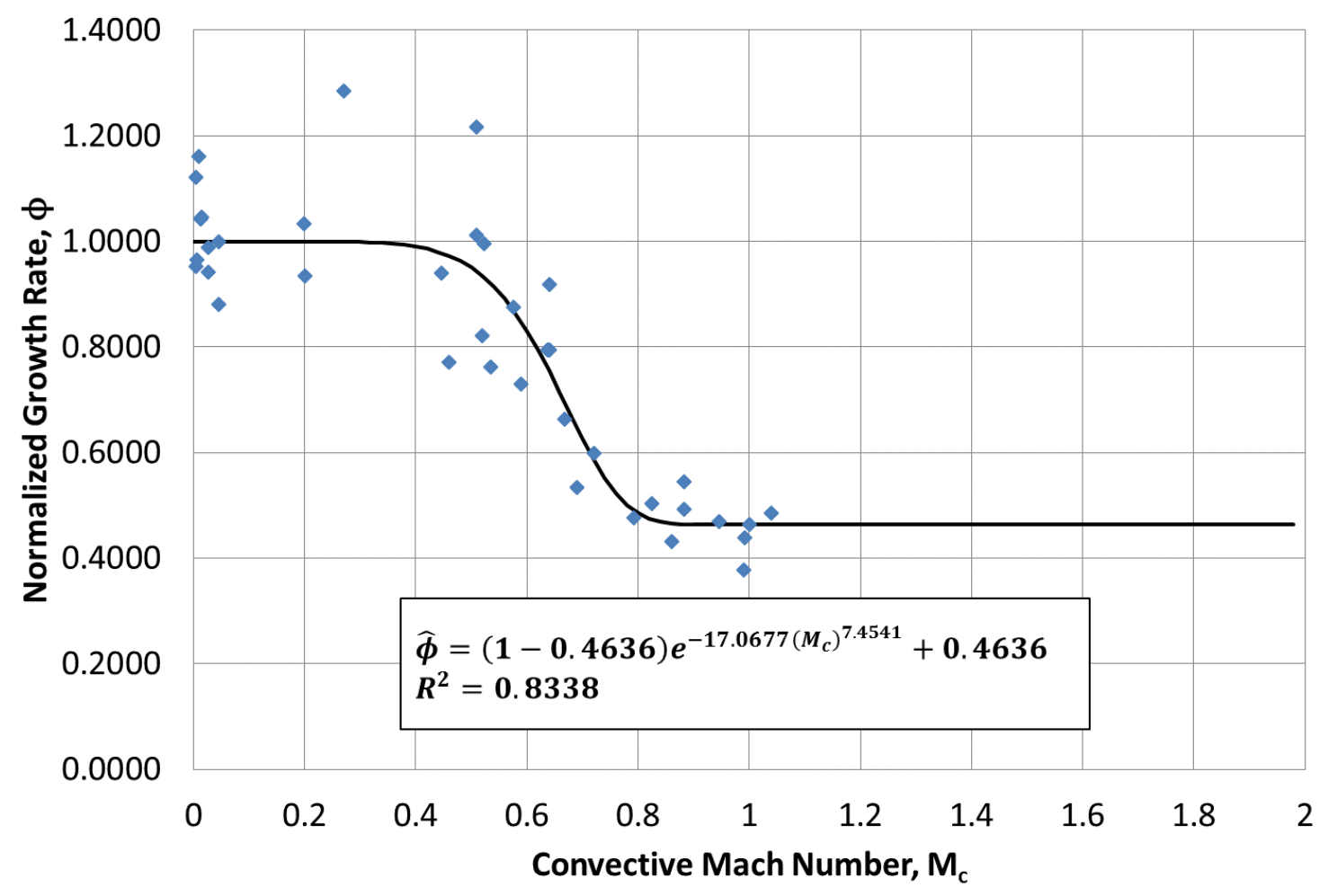

Figure 34. Data Set \#1 - Consistently normalized by incompressible growth rate model, $\sigma_{0}=10.3773$. 
The above modifications had a profound effect on the data set. Many cases that previously appeared to match the trend were shifted toward higher normalized growth rates. Some of these indicated normalized growth rates above unity at convective Mach numbers up to $M_{c} \approx 0.5$. On average, however, the data appear to have moved closer to one another, and the new model has a tighter fit. This is statistically evident by the higher value of $R^{2}=$ 0.8338 .

The histogram of residuals for Data Set \#1 is presented in Figure 35. When compared to Figure 29, this plot appears to be much more closely centered about zero. Furthermore, the bell curve shape is more prominent, although there is still a slight shift towards positive residuals.

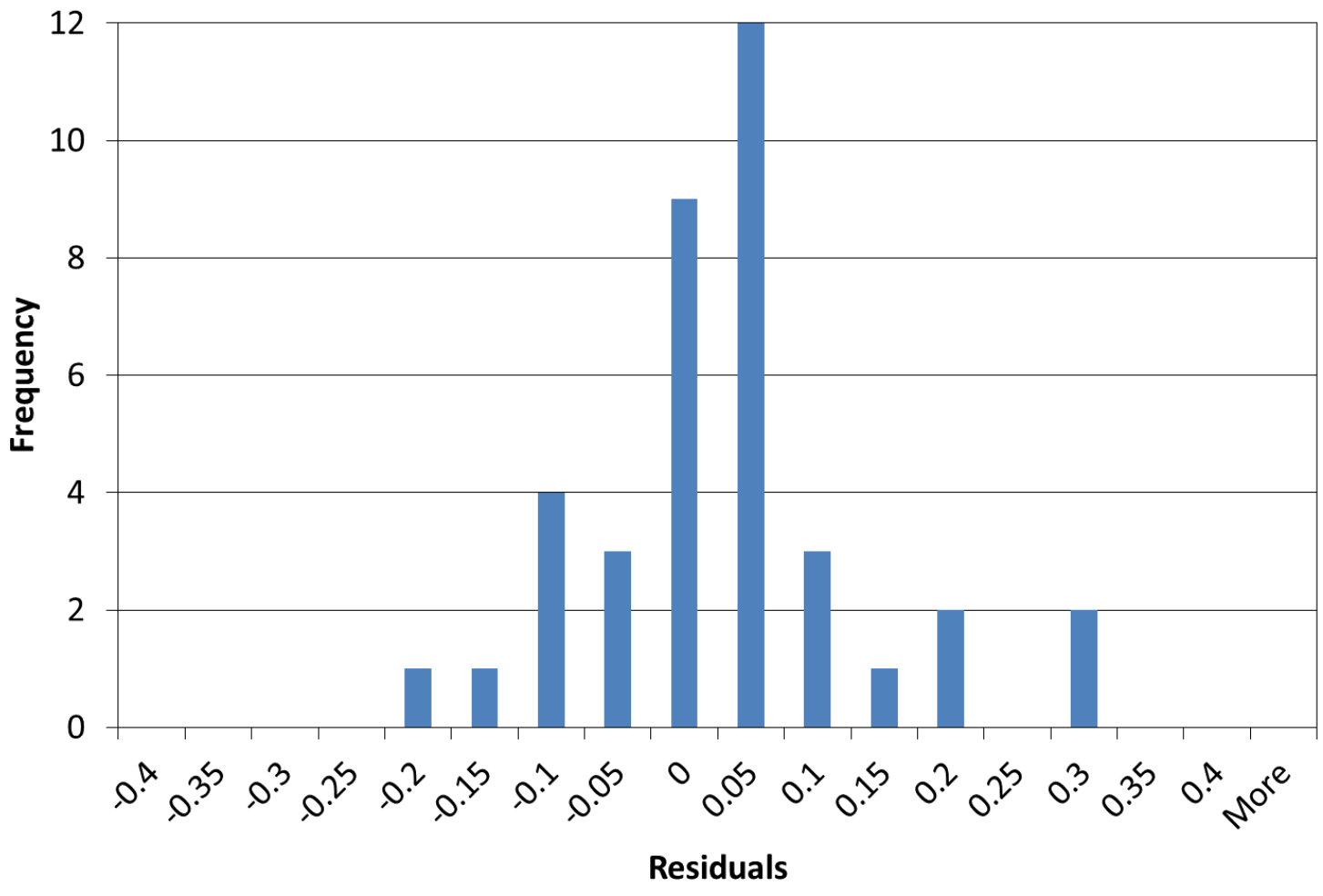

Figure 35. Data Set \#1 - Histogram of residuals. 


\subsection{Identification and Removal of Outliers}

Although the processes described in Section 4.3 effectively reduced a considerable portion of the scatter found in Data Set \#0 and provided a more reliable best-fit model, the resulting model could still have been affected by the contamination of possible outliers within the compressible regime. The effort described in this section identified any possible outliers present in Data Set \#1 using the same process that was applied in Section 4.3.1 to the incompressible data set. The cases that remain after the outliers were removed are grouped as Data Set \#2, the final data set.

The first boxplot, shown in Figure 36, identified three outliers. One of the two positiveresidual outlier cases was the $M_{c}=0.27$ case from Chinzei et al. (1986), which had previously been identified as anomalous by Barone et al. (2006) who noted pressure waves and/or freestream turbulence in the secondary stream that was visible in the Schlieren images. The second positive-residual outlier case was the vorticity thickness growth rate at $M_{c}=0.51$ from Samimy and Elliott (1990). The shear layer thickness growth rate associated with that same case is not necessarily affected by the anomalous report for vorticity thickness growth rate, although a physical explanation for the outlier could not be determined. The single negative-residual outlier was the $M_{c}=0.46$ case by Göebel and Dutton (1991), who noted that this case had streamwise deviations in freestream velocity as high as $13 \%$. 


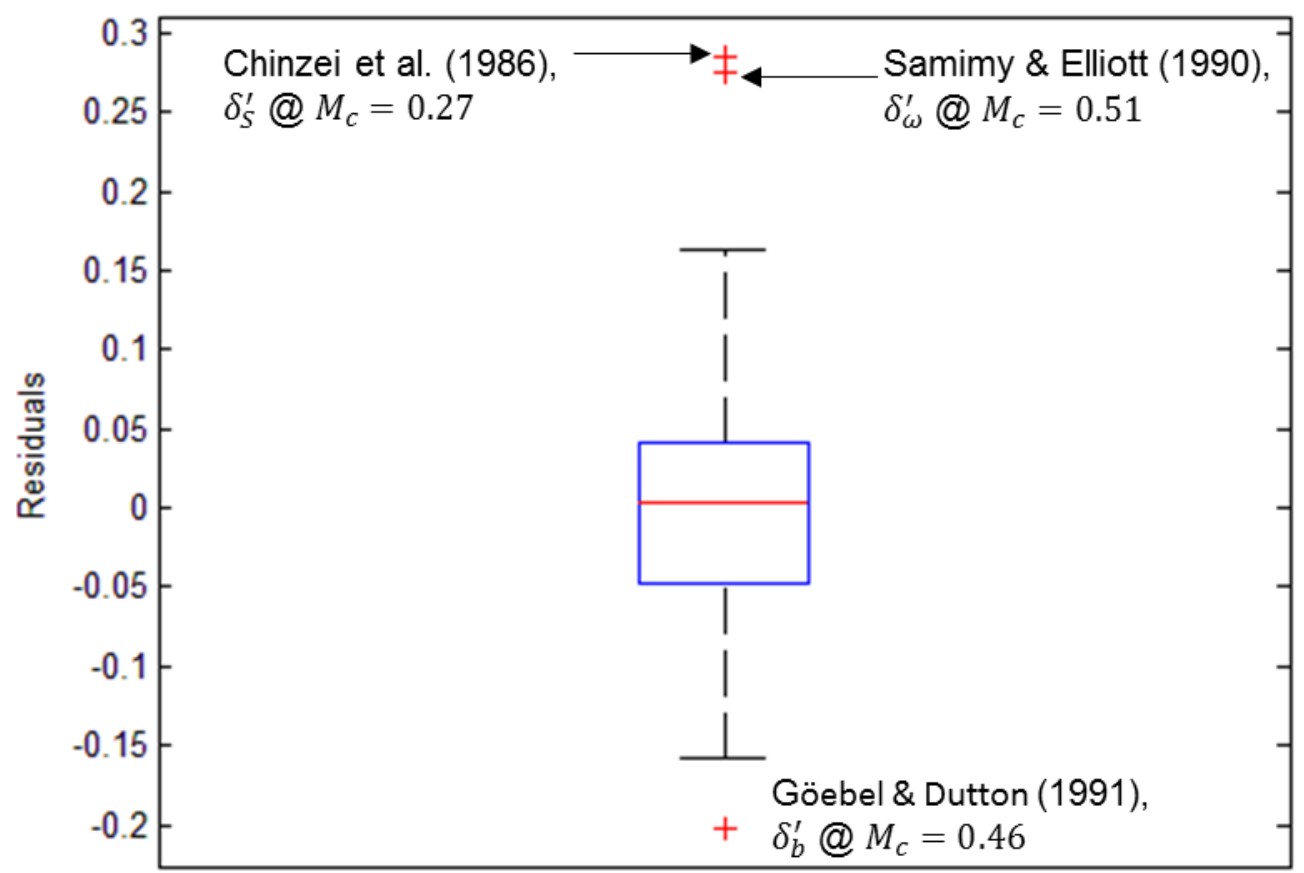

Figure 36. Boxplot of residuals for the first pass of outlier identification for Data Set \#1.

After the three outliers identified in Figure 36 were removed from the data set, the model parameters were re-evaluated to best fit the remaining data. The residuals associated with this new model are summarized in the boxplot in Figure 37. Since no further outliers were identified, this data set is finalized and named Data Set \#2. Had the new boxplot identified further outlier cases, the process would have been repeated. Figure 38 highlights all six of the identified outliers alongside Data Set \#2. The model included in this graph was fit to only Data Set \#2, and is the final model for the normalized growth rate. 


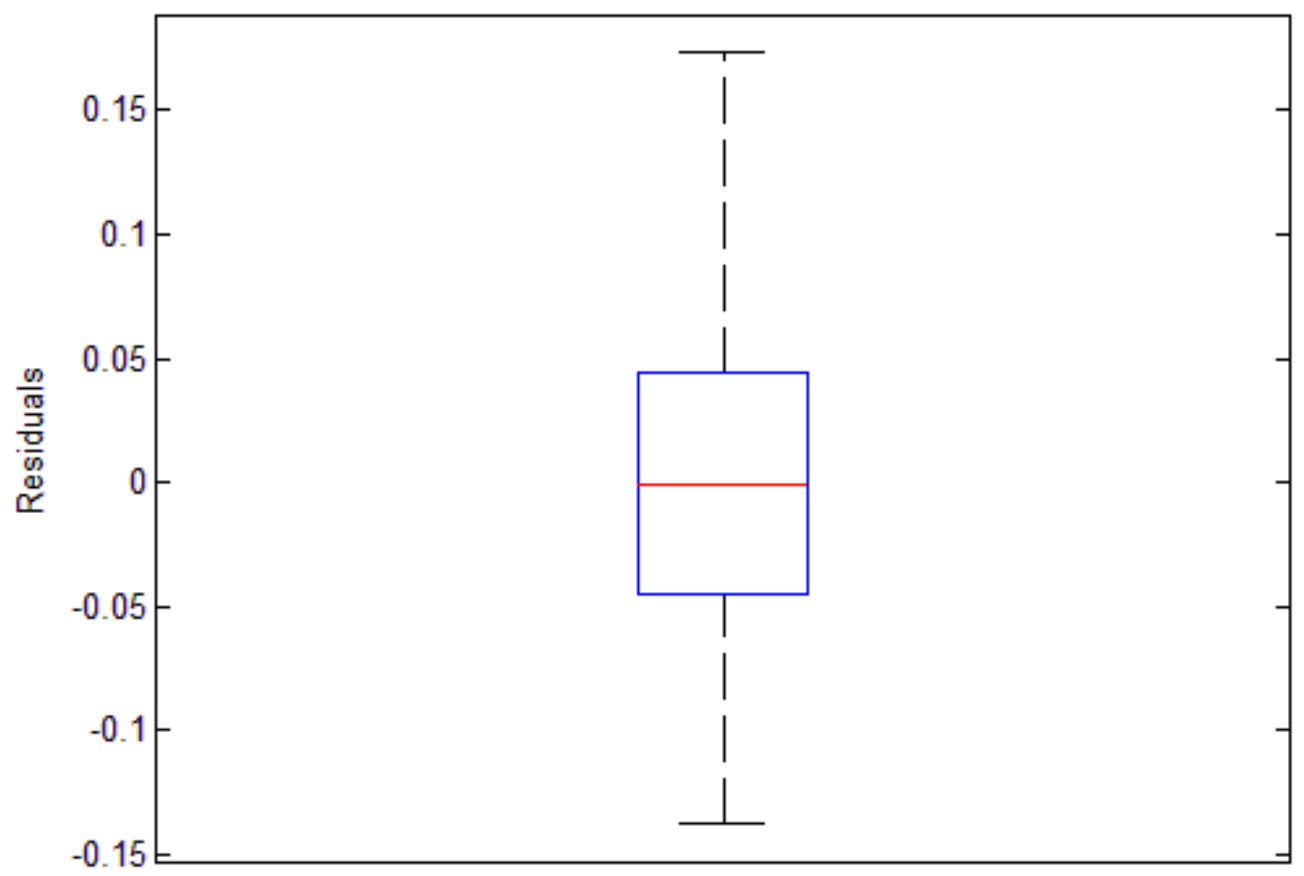

Figure 37. Boxplot of residuals for the second pass of outlier identification for Data Set \#2.

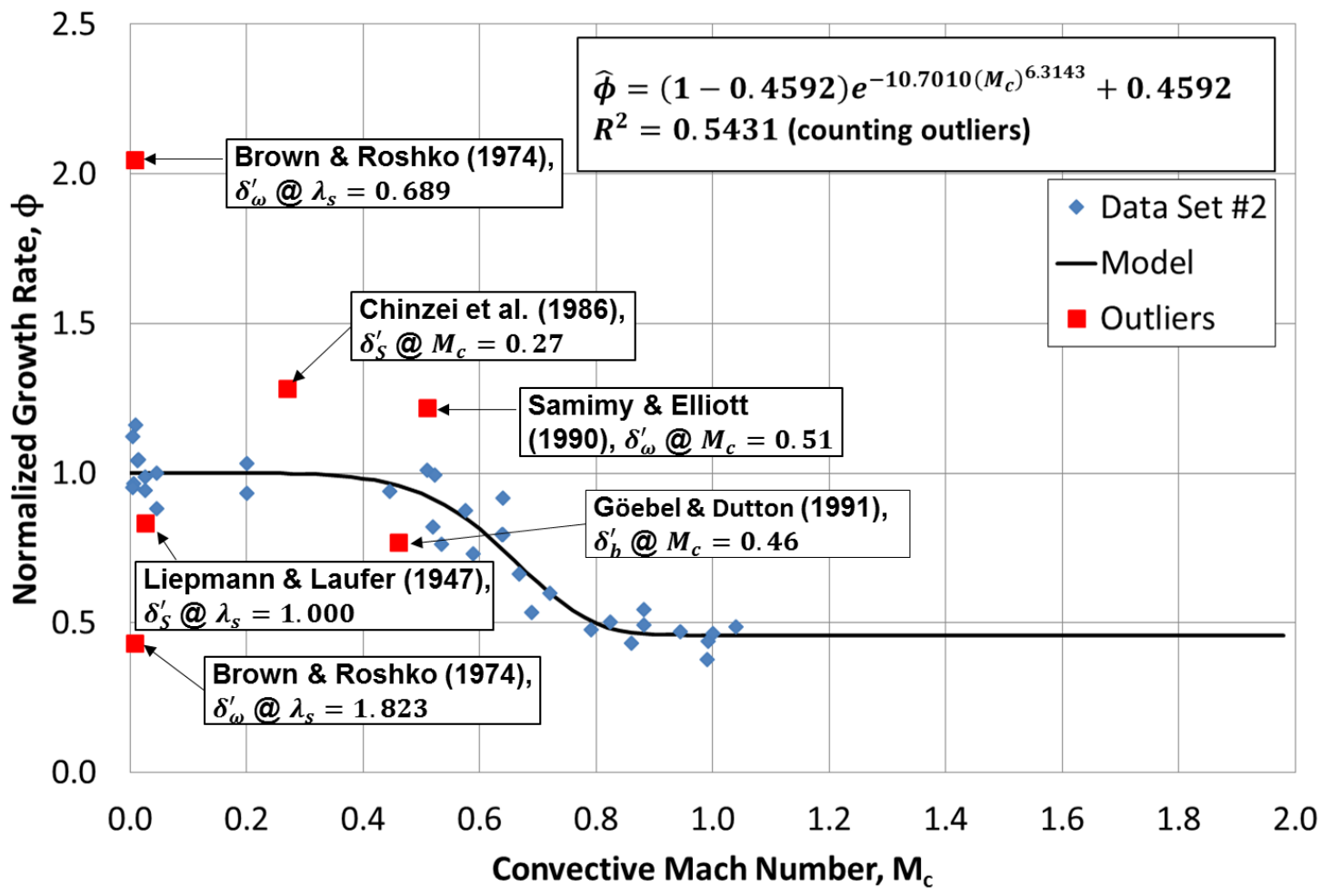

Figure 38. Six outliers shown alongside Data Set \#2. 


\subsection{Final Data Set and Fitted Model}

No further modifications were made to Data Set \#2. This final data set is shown with its best-fit model in Figure 39. For Data Set \#2, the best-fit model parameters are $A=0.4592$, $B=10.7010$, and $C=6.3143$. After the tuning and consistent application of the incompressible growth rate model and the removal of statistically identified outliers, the resulting data display considerably reduced variance. In fact, some of the largest residuals are found in the incompressible regime. The improved fit of the model is further supported by the respectable (although still not ideal) value of $R^{2}=0.9004$. In full equation form, the model is

$$
\widehat{\phi}=(1-0.4592) e^{-10.7010\left(M_{c}\right)^{6.3143}}+0.4592
$$

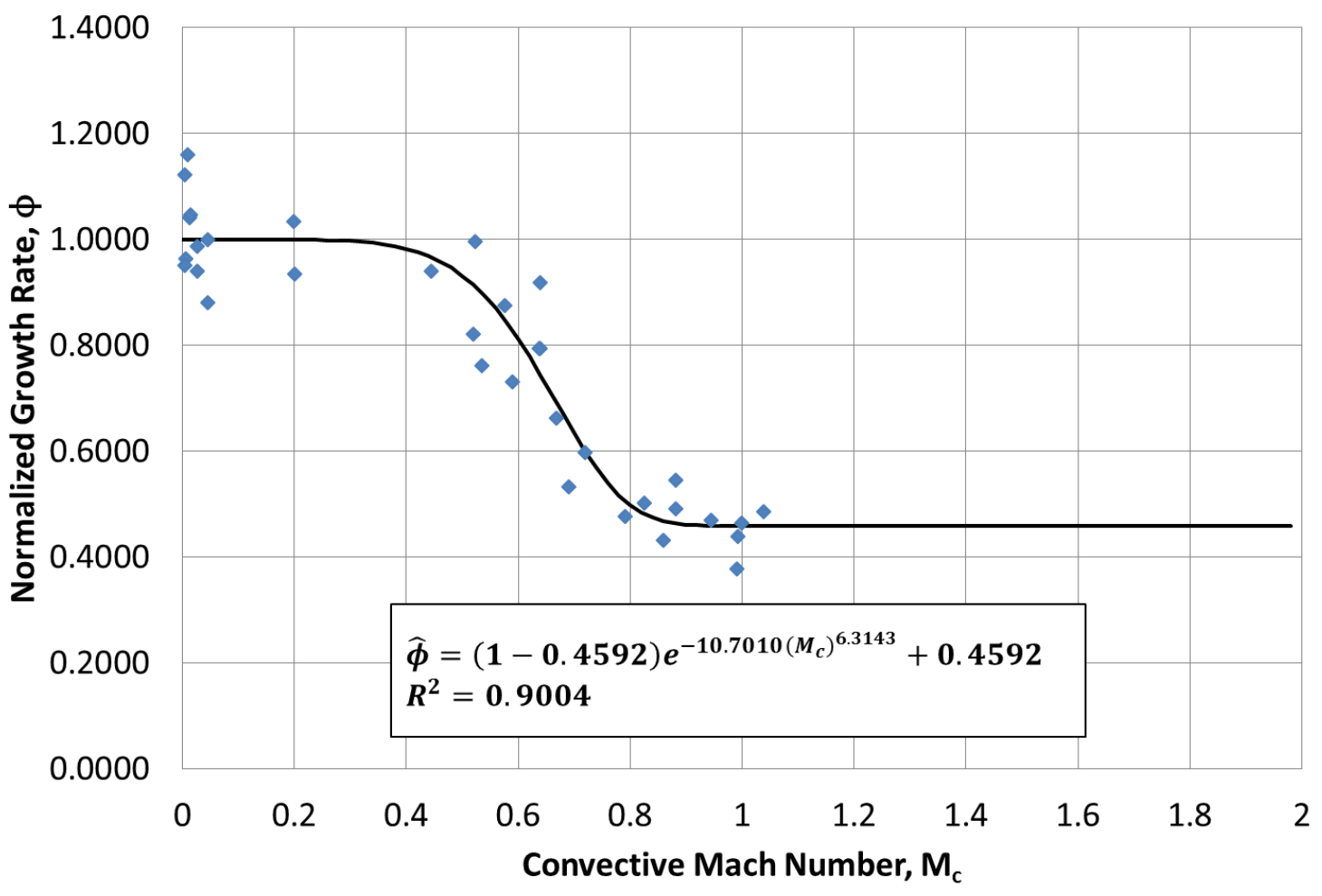

Figure 39. Data Set \#2 - Final data set - Outliers removed. 
The histogram of residuals for Data Set \#2, shown in Figure 40, exhibits good behavior. The overall shape of the curve exhibits normality. Although the residuals are still on average slightly skewed to the positive end, the magnitude of this shift is far improved from that of the baseline data set. Furthermore, the width of the histogram curve is much smaller than before. The largest residual in Data Set \#2 is only 0.173 , associated with the vorticity thickness growth rate measured by Samimy and Elliott (1990) for their underexpanded case.

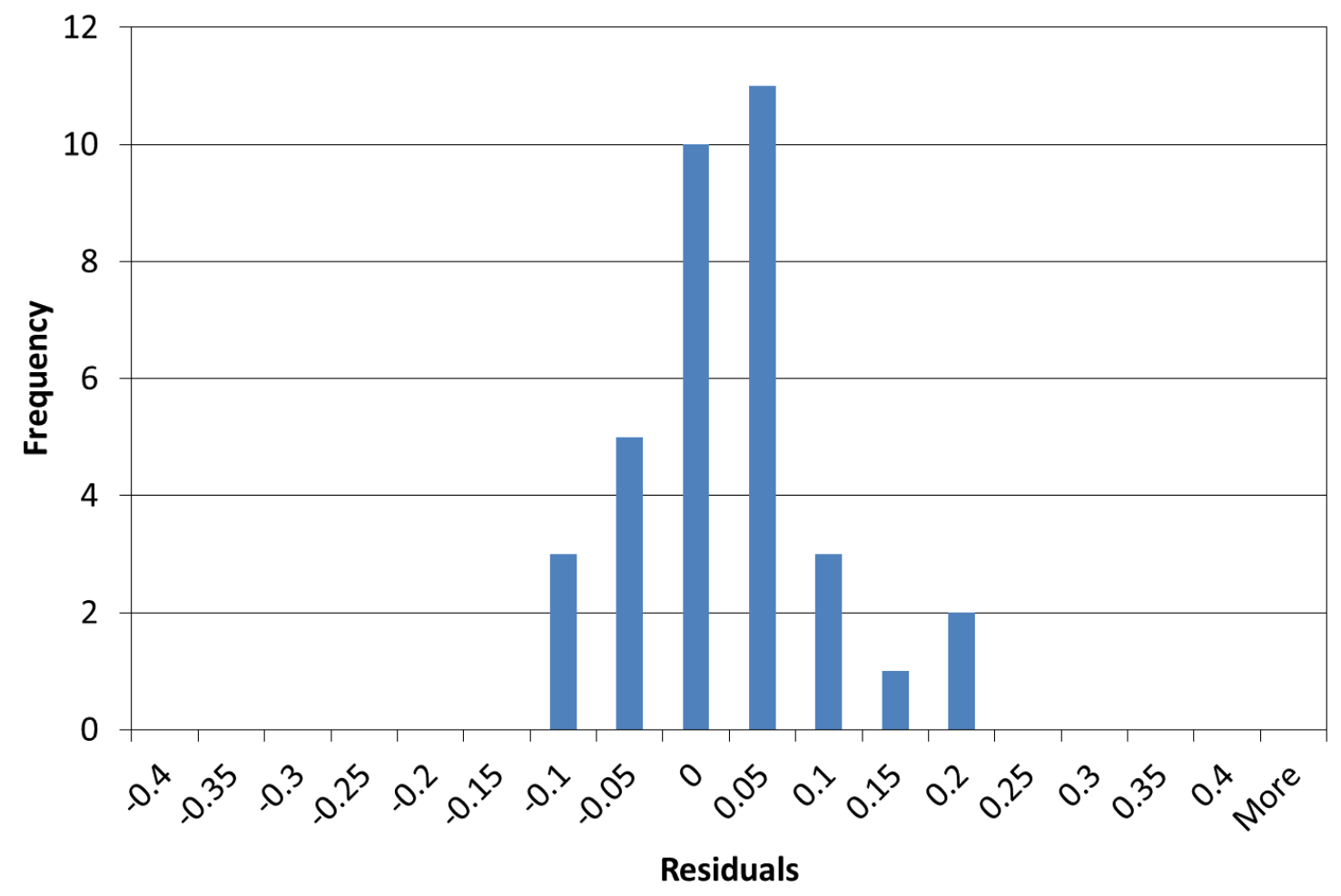

Figure 40. Data Set \#2 - Histogram of residuals.

A plot of percent difference between Data Set \#2 and its associated model is shown in Figure 41 as yet another diagnostic tool. From this plot, it can be easily seen that the model is accurate to roughly $\pm 20 \%$. 


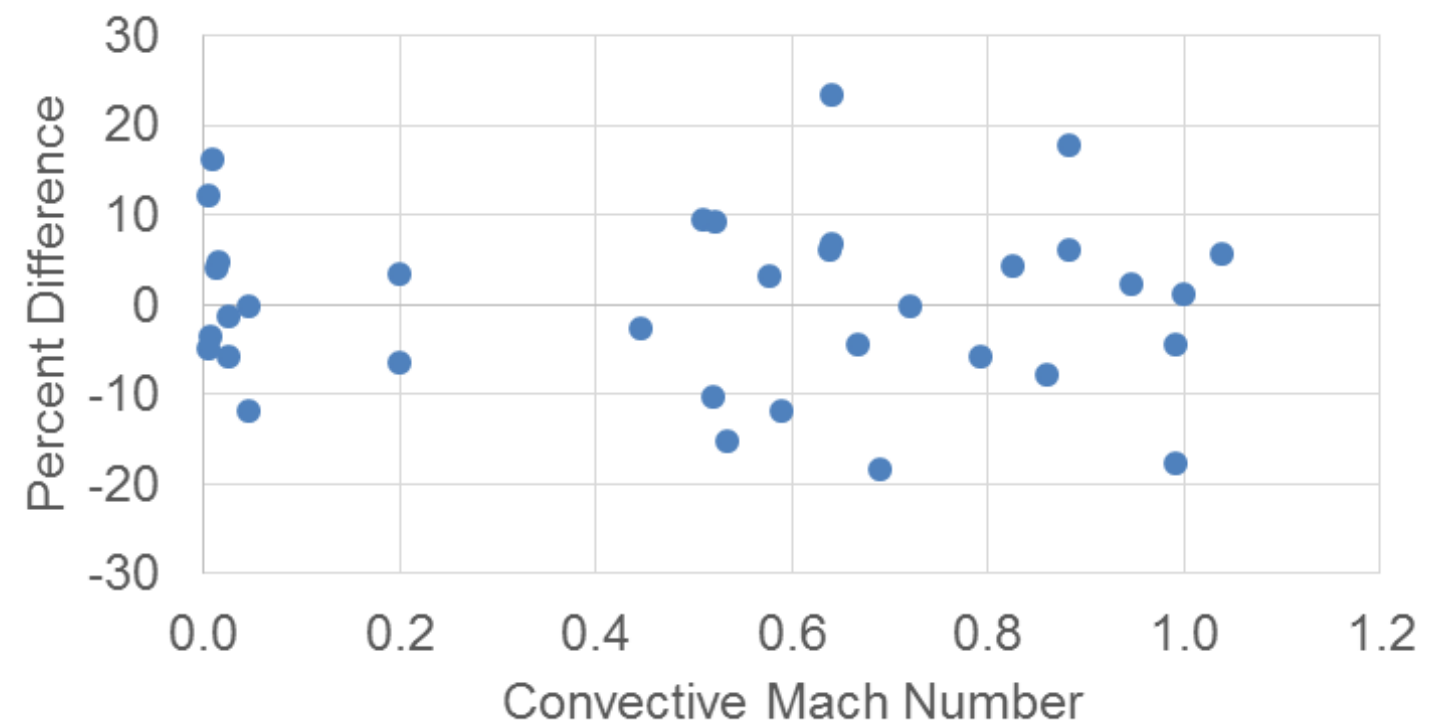

Figure 41. Percent difference between Data Set \#2 and its associated model.

Although this data set and model are considerably more trustworthy than those presently available in the literature, there is still room for improvement. First and foremost, there is a clear need for more data. The qualification rubric discussed in Section 2.2 was a necessary evil in this regard. It effectively prohibited numerous studies from inclusion within the baseline data set in order to ensure that the model was uncontaminated by additional parameters. Further studies that prove some of the requirements to be unnecessary could considerably increase the size of the data set. Moreover, a study that validates a functional relationship between the velocity-based mixing layer growth rates and those of either the visual or density-based definitions would have a similar (and perhaps more profound) effect. To validate such a relationship, one would need to test it for both incompressible and compressible cases. Fortunately, the process described in Section 4 should easily accommodate larger data sets; a new yet comparable model can be fitted with each forthcoming study. 
Another shortcoming of the present data set is the utter lack of error analysis for the individual data points. In comparison to the visual thickness data set, wherein 18 of the 27 cases were reported with uncertainty of the observed mixing layer growth rate, only 1 of the 41 velocity-based growth rates was reported with uncertainty information. It is an embarrassment to the original author and the research community as a whole when the uncertainty of the data acquisition is not propagated to the ultimate output of a report. 


\section{Conclusions}

The work discussed herein introduced an improved parametric model for the growth rate of turbulent mixing layers. The model was made using statistical regression of data from a collection of 162 related references. A data qualification rubric was established and used to narrow the variety of studies to the manageable subset of planar, spatial, nonreacting, free shear layers that were not affected by artificial mixing enhancement techniques. In keeping with existing practice, the model is two-fold; the growth rate $\left(\delta^{\prime}\right)$ can be predicted by determining an incompressible growth rate estimate $\left(\delta_{0}^{\prime}\right)$ and a compressibility factor (i.e. normalized growth rate, $\phi$ ) such that $\delta^{\prime}=\phi \delta_{0}^{\prime}$. The model for the incompressible growth rate was derived from the error function velocity profile for three different velocitybased mixing layer thickness definitions and tuned to match the incompressible subset of cases from the database. Afterwards, a parametric model for the normalized growth rate as a function of the convective Mach number $\left(M_{c}\right)$ was optimized to best fit the entire viable database. The two parts of the model are summarized in the following equations:

$$
\begin{gathered}
\phi=\frac{\delta^{\prime}}{\delta_{0}^{\prime}} \cong(1-0.4592) e^{-10.7010\left(M_{c}\right)^{6.3143}}+0.4592 \\
\delta_{0}^{\prime}=C_{\delta} \frac{(1+r)(1+r \sqrt{s})}{1+\sqrt{s}}=C_{\delta} \lambda_{s} \\
C_{b} \cong \frac{1.8124}{\sigma_{0}}, \quad C_{S} \cong \frac{1.4923}{\sigma_{0}}, \quad C_{\omega} \cong \frac{\sqrt{\pi}}{\sigma_{0}} \\
\sigma_{0}=10.3773
\end{gathered}
$$

Both aspects of the model displayed improved goodness of fit, with $R^{2}=0.9856$ for the incompressible growth rate model (up from baseline $R^{2}=0.5603$ ) and $R^{2}=0.9004$ for the normalized growth rate model (up from baseline $R^{2}=0.5444$ ). These improvements are 
attributed to the following theoretical and statistical findings which were reflected in the modeling process:

- $\quad$ The greatest impact on the $R^{2}$ values was achieved through the identification and removal of six outlier observations from Liepmann and Laufer (1947), Brown and Roshko (1974), Chinzei et al. (1986), Samimy and Elliott (1990), and Göebel and Dutton (1991).

- Despite contemporary thought, a systematic difference was found between normalized growth rates for the different thickness definitions based on the velocity profile, the density profile, and visualizations. Only the velocity-based thicknesses were carried into the modeling process because of the existence of the error function velocity profile, which was paramount to the development of the incompressible growth rate model. No comparable models presently exist for density profiles or visualization techniques.

- The process used by Slessor to re-evaluate the incompressible growth rates was shown to utilize an inappropriate assumption. If the assumption is disregarded, the benefits of Slessor's compressibility parameter $\left(\Pi_{c}\right)$ are substantially reduced.

- The derivation for the convective Mach number was assessed for the errors introduced through assumptions and mathematical simplifications. The parameter was deemed generally acceptable with errors smaller than $\pm 2 \%$ for most scenarios, however caution is recommended for rare scenarios in which errors can exceed $\pm 10 \%$. In particular, the model becomes invalid if strong pressure gradients exist. 
- The normalized growth rate model from Dimotakis (1991a) was generalized into a parametric function, and its adequacy as a model for the present data was confirmed. The function met all four sensitivity criteria that were identified.

- Considerable inconsistencies regarding the application of an incompressible growth rate model as a normalizing agent were recognized throughout the literature. The data from Chinzei et al. (1986) served as a case example of the extent to which later authors effectively modified the representation of the observed growth rates through inconsistent normalization. The present analysis resolved this issue by normalizing the entire set of observed growth rates by a single incompressible growth rate model.

The logical continuation of this effort would be to somehow add more data to the set being modeled, which can be accomplished in a number of ways. The obvious method would involve novel studies that target parameter combinations that are not well populated in the present database. Care should be taken to ensure that such studies meet the criteria listed in the data qualification rubric. Another approach could be an even more thorough search of the literature, perhaps focusing on the older, more difficult to obtain studies. Of course, such an approach would inevitably suffer from diminishing returns. Theoretical investigations could also be used to achieve the desired effect by somehow providing justification for the relaxation of one or more of the criteria used to isolate the pertinent parameters. As data both new and old are added to the database, the systematic approach described herein will provide a simple, effective, and robust method for refining the turbulent mixing layer growth rate model. 


\section{References}

ALVI, F. S., KROTHAPALLI, A. \& WASHINGTON, D. 1995a An experimental study of a compressible countercurrent turbulent shear layer. AIAA Paper 95-0580.

ALVI, F. S., KROTHAPALLI, A. \& WASHINGTON, D. 1995b Fluctuating pressure measurements in a highly compressible countercurrent turbulent shear layer. AIAA Paper 95-2175.

ASO, S., INOUE, K., YAMAGUCHI, K. \& TANI, Y. 2009 A study on supersonic mixing by circular nozzle with various injection angles for air breathing engine. Acta Astronautica 65, 687-695.

BARONE, M. F., OBERKAMPF, W. L. \& BLOTTNER, F. G. 2006 Validation case study: Prediction of compressible turbulent mixing layer growth rate. AIAA J. 44(7), 14881497.

BARRE, S., BRAUD, P., CHAMBRES, O. \& BONNET, J. P. 1997 Influence of inlet pressure conditions on supersonic turbulent mixing layers. Exp. Therm. Fluid Sci. 14 (1), 68-74.

BIRCH, S. F. \& EGGERS, J. M. 1973 Free turbulent shear flows. NASA Tech. Rep. SP321.

BOGDANOFF, D. W. 1983 Compressibility effects in turbulent shear layers. AIAA J. 21, 926-927.

BRADSHAW, P. 1966 The effect of initial conditions on the development of a free shear layer. J. Fluid Mech. 26, 225-236.

BROWN, G. L. \& ROSHKO, A. 1974 On density effects and large structure in turbulent mixing layers. J. Fluid Mech. 64, 775-816.

BRUMMUND, U. \& NUDING, J. R. 1997 Interaction of a compressible shear layer with shock waves - An experimental study. AIAA Paper 97-0392.

CHINZEI, N., MASUA, G., KOMURO, T., MURAKAMI, A. \& KUDOU, K. 1986 Spreading of two-stream supersonic turbulent mixing layers. Phys. Fluids 29, $1345-1347$.

CHRISTOPOULOS, A. \& LEW, M. J. 2000 Beyond eyeballing: Fitting models to experimental data. Crit. Rev. Biochem. Mol. Biol. 35(5), 359-391.

DAY, M. J., REYNOLDS, W. C. \& MANSOUR, N. N. 1998 The structure of the compressible reacting mixing layer: insights from linear stability analysis. Phys. Fluids 10 (4), 993-1007

DIMOTAKIS, P. E. 1986 Two-dimensional shear-layer entrainment. AIAA J. 24, 17911796. 
DIMOTAKIS, P. E. 1991a Turbulent free shear layer mixing and combustion. In High Speed Flight Propulsion Systems. Prog. Astro. Aero. 137, pp. 265-340.

DIMOTAKIS, P. E. 2005 Turbulent mixing. Annu. Rev. Fluid Mech. 37, 329-356

DOTY, M. J. \& MCLAUGHLIN, D. K. 2000 Experiments on Mach-wave interactions in a compressible shear layer. AIAA J. 38(10), 1871-1878.

ERDOS, J., TAMANGO, J., BAKOS, R. \& TRUCCO, R. 1992 Experiments on shear layer mixing at hypervelocity conditions. AIAA Paper 92-0628.

FREUND, J. B., LELE, S. K. \& MOIN, P. 1997 Direct simulation of a supersonic round turbulent shear layer. AlAA Paper 97-0760.

FREUND, J. B., LELE, S. K. \& MOIN, P. 2000 Compressibility effects in a turbulent annular mixing layer. Part 1. Turbulence and growth rate. J. Fluid Mech. 421, 229-267.

GATSKI, T. B. \& BONNET, J. P. 2009 Compressibility, turbulence and high speed flows, 1st ed. Elsevier Science. ISBN: 978-0-08-044565-6.

GÖEBEL, S. G. \& DUTTON, J. C. 1991 Experimental study of compressible turbulent mixing layers. AIAA J. 29, 538-546.

HODGE, V. J. \& AUSTIN, J. 2004 A survey of outlier detection methodologies. Artif. Intell. Rev. 22, 85-126.

ISLAND, T. C., URBAN, W. D. \& MUNGAL, M. G. 1998 Mixing enhancement in compressible shear layers via sub-boundary layer disturbances. Phys. Fluids 10(4), 1008-1020.

KLINE, S. J., CANTWELL, B. J. \& LILLEY, G. M. 1983 The 1980-81 AFOSR-HTTMStanford conference on complex turbulent flows: Comparison of computation and experiment - Volume I. AFOSR-TR-83-1001.

LIEPMANN, H. W. \& LAUFER, J. 1947 Investigation of free turbulent mixing. NACA Tech. Note, no. 1257.

MAYDEW, R. C. \& REED, J. F. 1963 Turbulent mixing of axisymmetric compressible jets (in the half-jet region) with quiescent air. Sandia Corp. Report SC-4764(RR).

NAUGHTON, J. W., CATTAFESTA, L. N. \& SETTLES, G. S. 1997 An experimental study of compressible turbulent mixing enhancement in swirling jets. J. Fluid Mech. 330, 271-305.

PANTANO, C. \& SARKAR, S. 2002 A study of compressibility effects in the high-speed turbulent shear layer using direct simulation. J. Fluid Mech. 451, 329-371.

PAPAMOSCHOU, D. 1995 Evidence of shocklets in a counterflow supersonic shear layer. Phys. Fluids 7, 233-235.

PAPAMOSCHOU, D. \& ROSHKO, A. 1988 The compressible turbulent shear layer: an experimental study. J. Fluid Mech. 197, 453-477. 
PETRIE, H. L., SAMIMY, M. \& ADDY, A. L. 1985 A study of compressible turbulent free shear layers using laser Doppler velocimetry. AIAA Paper 85-0177.

RAMASWAMY, M. \& LOTH, E. 1996 Tone excitation of a supersonic bounded shear layer. AIAA J. 34(10), 1997-2004.

REICHERT, R. S. \& BIRINGEN, S. 2006 Numerical simulation of compressible plane jets. Mech. Res. Commun. 34, 249-259.

SAMIMY, M. \& ELLIOTT, G. S. 1990 Effects of compressibility on the characteristics of free shear layers. AIAA J. 28, 439-445.

SCHLICHTING, H. 1979 Boundary-Layer Theory, 7th ed. McGraw - Hill, New York.

SLESSOR, M. D. 1998 Aspects of turbulent-shear-layer dynamics and mixing. PhD thesis, California Institute of Technology.

SMITS, A. J. \& DUSSAUGE, J. P. 2006 Turbulent shear layers in supersonic flow. Springer. ISBN 0387263055.

STRYKOWSKI, P. J., KROTHAPALLI, A. \& JENDOUBI, S. 1996 The effect of counterflow on the development of compressible shear layers. J. Fluid Mech. 308, 63-96.

STRYKOWSKI, P. J., KROTHAPALLI, A. \& WISHART, D. 1993 Enhancement of mixing in high-speed heated jets using a counterflowing nozzle. AIAA J. 31, 2033-2038.

THUROW, B. S., JIANG, N., KIM, J. H., LEMPERT, W. \& SAMIMY, M. 2008 Issues with measurements of the convective velocity of large-scale structures in the compressible shear layer of a free jet. Phys. Fluids 20, 066101.

VREMAN, A. W., SANDHAM, N. D. \& LUO, K. H. 1996 Compressible mixing layer growth rate and turbulence characteristics. J. Fluid Mech. 320, 235-258.

WYGNANSKI, I. \& FIEDLER, H. E. 1970 The two-dimensional mixing region. J. Fluid Mech. 41, 327.

ZHOU, Q., HE, F. \& SHEN, M. Y. 2012 Direct numerical simulation of a spatially developing compressible plane mixing layer: flow structures and mean flow properties. J. Fluid Mech. 711, 437-468. 


\section{Appendix A: Complete Library Reference List}

The following multi-page table includes all citations for all of the articles, papers, textbooks, etc. that were identified for the turbulent mixing layer library. The references are organized alphabetically. The column on the right identifies which of these references were collected and processed for the present database. Undoubtedly, several applicable references exist beyond the reaches of this library. It is the hope of this author that the list provided here can serve as a foundation for other authors to make even more extensive libraries.

Citation

Acquired

ABE, T. \& FUNABIKI, K. 1992 Effect of streamwise pressure gradient on Yes the supersonic mixing layer. AIAA J. 30(10), 2564-2566.

AGARD 1998 A selection of test cases for the validation of large-eddy Yes simulations of turbulent flows. AGARD Advisory Report No. 345.

ALVI, F. S., KROTHAPALLI, A. \& WASHINGTON, D. 1995a An Yes experimental study of a compressible countercurrent turbulent shear layer. AIAA Paper 95-0580.

ALVI, F. S., KROTHAPALLI, A. \& WASHINGTON, D. 1995b Fluctuating pressure measurements in a highly compressible countercurrent turbulent shear layer. AIAA Paper 95-2175.

ASO, S., INOUE, K., YAMAGUCHI, K. \& TANI, Y. 2009 A study on Yes supersonic mixing by circular nozzle with various injection angles for air breathing engine. Acta Astronautica 65, 687-695.

AUPOIX, B. 2004 Modelling of compressibility effects in mixing layers. J. Yes Turbulence 5, N7.

BARLOW, R. S., FOURGUETTE, D. C., MUNGAL, M. G. \& DIBBLE, R. W. Yes 1992 Experiments on the structure of an annular compressible reacting shear layer. AIAA J. 30(9), 2244-2251.

BARONE, M. F., OBERKAMPF, W. L. \& BLOTTNER, F. G. 2006 Validation Yes case study: Prediction of compressible turbulent mixing layer growth rate. AIAA J. 44(7), 1488-1497.

BARRE, S. 1994 Estimate of convective velocity in a supersonic turbulent Yes mixing layer. AIAA J. 32(1), 211-213.

BARRE, S., QUINE, C. \& DUSSAUGE, J. P. 1994 Compressibility effects Yes on the structure of mixing layers: Experimental results. J. Fluid Mech. 259, 47-78.

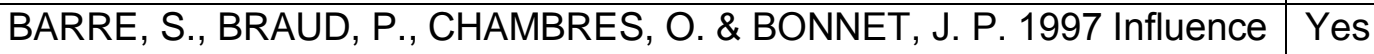
of inlet pressure conditions on supersonic turbulent mixing layers. Exp. Therm. Fluid Sci. 14 (1), 68-74.

BELL, J. H. \& MEHTA, R. D. 1990 Development of a two-stream mixing yes layer with tripped and untripped boundary layers. AIAA J. 28, 20342042 
BIRCH, S. F. \& EGGERS, J. M. 1973 Free turbulent shear flows. NASA yes Tech. Rep. SP-321.

BLOHM, A., LEMPERT, W., SAMIMY, M. \& THUROW, B. 2006 A study of yes convective velocity in supersonic jets using $\mathrm{MHz}$ rate imaging. AIAA Paper 2006-45.

BODI, K. V. R. 2005 Large eddy simulations of compressible mixing layers. $y e s$ M.Sc. Thesis, India Institute of Science.

BOGDANOFF, D. W. 1983 Compressibility effects in turbulent shear layers. yes AIAA J. 21, 926-927.

BOGDANOFF, D. W. 1984 Interferometric measurement of heterogeneous yes shear-layer spreading rates. AIAA J. 22, 1550.

BONNET, J. P., DEBISSCHOP, J. R. \& CHAMBERS, O. 1993 Experimental yes studies of the turbulent structure of supersonic mixing layers. AIAA Paper 93-0217.

BOUZADA, B. E., WIJEYAKULASURIYA, S. D. \& NALIM, M. R. 2010 yes Improved compressible-jet shear-layer simulations using RANS models for steady confined jets. AIAA Paper 2010-4746.

BOWERSOX, R. D. \& SCHETZ, J. A. 1995 Measurements of compressible yes turbulent flow structure in a supersonic mixing layer. AIAA J. 33(11), 2101-2106.

BRADSHAW, P. 1966 The effect of initial conditions on the development of yes a free shear layer. J. Fluid Mech. 26, 225-236.

BRADSHAW, P. 1977 Compressible turbulent shear layers. Ann. Rev. Fluid Yes Mech. 9, 33-54.

BROWN, G. L. \& ROSHKO, A. 1974 On density effects and large structure yes in turbulent mixing layers. J. Fluid Mech. 64, 775-816.

BRUMMUND, U. \& MESNIER, B. 1999 Experimental study of compressible no mixing layers. In 14th Int. Symp. On Airbreathing Engines.

BRUMMUND, U. \& NUDING, J. R. 1997 Interaction of a compressible shear yes layer with shock waves - An experimental study. AIAA Paper 97-0392.

BUSH, R. H., CULVER, H. C. M., WEISSBEIN, D. \& GEORGIADIS, N. J. no 2013 Low velocity difference thermal shear layer mixing rate measurements. AIAA Paper 2013-1074.

BUTTSWORTH, D. R., MORGAN, R. G. \& JONES, T. V. 1995 A gun tunnel yes investigation of hypersonic free shear layers in a planar duct. J. Fluid Mech. 299, 133-152.

CHAMBRES, O., BARRE, S. \& BONNET, J. 1998 Detailed turbulent no characteristics of a highly compressible supersonic turbulent plane mixing layer. J. Fluid Mech. Submitted.

CHINZEI, N., MASUA, G., KOMURO, T., MURAKAMI, A. \& KUDOU, K. yes 1986 Spreading of two-stream supersonic turbulent mixing layers. Phys. Fluids 29, $1345-1347$.

CLEMENS, N. T. \& MUNGAL, M. G. 1992a Effects of sidewall disturbances yes on the supersonic mixing layer. J. Propul. Power 8, 249.

CLEMENS, N. T. \& MUNGAL, M. G. 1992b Two- and three-dimensional yes effects in the supersonic mixing layer. AIAA J. 30, 973-981.

CLEMENS, N. T. \& MUNGAL, M. G. 1995 Large-scale structure and yes entrainment in the supersonic mixing layer. J. Fluid Mech. 284, 171216. 
CLEMENS, N. T. \& PAUL, P. H. 1995 Scalar measurements in yes compressible axisymmetric mixing layers. Phys. Fluids 7, 1071-1081.

CLEMENS, N. T., PAUL, P. H., MUNGAL, M. G. \& HANSON, R. K. 1991 yes Scalar mixing in the supersonic shear layer. AIAA Paper 91-1720.

CLEMENS, N. T., PETULLO, S. P. \& DOLLING, D. S. 1996 Large-scale yes structure evolution in supersonic interacting shear layers. AIAA J. 34(10), 2062-2070.

COTTRELL, D. C. \& PLESNIAK, M. W. 2001 Sensitivity of mixed subsonic- yes supersonic turbulent mixing layers. J. Propul. Power 17(4), 823-835.

DAY, M. J., REYNOLDS, W. C. \& MANSOUR, N. N. 1998 The structure of yes the compressible reacting mixing layer: insights from linear stability analysis. Phys. Fluids 10 (4), 993-1007.

DEBIĖVE, J. F., DUPONT, P., LAURENT, H., MENAA, M. \& DUSSAUGE, yes J. P. 2000 Compressibility and structure of turbulence in supersonic shear flows. Eur. J. Mech. B - Fluids 19, 597-614.

DEBISSCHOP, J. R. \& BONNET, J. P. 1993 Mean and fluctuating velocity no measurements in supersonic mixing layers. In Engineering Turbulence Modeling and Experiments 2, (ed. W. Rodi and F. Martelli) Elsevier.

DEBISSCHOP, J. R., CHAMBRES, O. \& BONNET, J. P. 1994 Velocity-field yes characteristics in supersonic mixing layers. Exp. Therm. Fluid Sci. 9, $147-155$.

DELARUE, B. J. \& POPE, S. B. 1997 Application of PDF methods to yes compressible turbulent flows. Am. Inst. Phys. 9(9), 2704-2716.

DIMOTAKIS, P. E. 1986 Two-dimensional shear-layer entrainment. AIAA J. yes 24, 1791-1796.

DIMOTAKIS, P. E. 1991a Turbulent free shear layer mixing and yes combustion. In High Speed Flight Propulsion Systems. Prog. Astro. Aero. 137, pp. 265-340.

DIMOTAKIS, P. E. 1991b On the convection velocity of turbulent structures yes in supersonic shear layers. AIAA Paper 91-1724.

DIMOTAKIS, P. E. 2005 Turbulent mixing. Annu. Rev. Fluid Mech. 37, 329- yes 356.

DOTY, M. J. \& MCLAUGHLIN, D. K. 2000 Experiments on Mach-wave yes interactions in a compressible shear layer. AIAA J. 38(10), 1871-1878.

DUTTON, J. C., BURR, R. F., GOEBEL, S. G. \& MESSERSMITH, N. L. yes 1990 Compressibility and mixing in turbulent free shear layers. Preprints for the Symposium on Turbulence, -A22.12.

ELLIOTT, G. S. \& SAMIMY, M. 1990 Compressibility effects in free shear yes layers. Phys. Fluids A 2, 1231-1240.

ELLIOTT, G. S., SAMIMY, M. \& REEDER, M. F. 1990 Pressure-based real- yes time measurements in compressible free shear layers. AIAA Paper 90-1980.

ELLIOTT, G. S., SAMIMY, M. \& ARNETTE, S. A. 1992 Study of yes compressible mixing layers using filtered Rayleigh scattering based visualizations. AIAA J. 30(10), 2567-2569.

ELLIOTT, G. S., SAMIMY, M. \& ARNETTE, S. A. 1995 The characteristics yes and evolution of large-scale structures in compressible mixing layers. Phys. Fluids 7, 864-876. 
ELZAWAWY, A., BRAVO, L., ANDREOPOULOS, Y. \& WATKINS, C. B. yes 2007 Compressibility and density effects in free subsonic jet flows: Three dimensional PIV measurements of turbulence. AIAA Paper 2007-3858.

ERDOS, J., TAMANGO, J., BAKOS, R. \& TRUCCO, R. 1992 Experiments yes on shear layer mixing at hypervelocity conditions. AIAA Paper 920628.

FOURGUETTE, D. C., DIBBLE, R. W. \& MUNGAL, M. G. 1990 Time yes evolution of the shear layer of a supersonic axisymmetric jet at matched conditions. AIAA J. 10, 157-166.

FOURGUETTE, D. C., MUNGAL, M. G., BARLOW, R. S. \& DIBBLE, R. W. yes 1991a Concentration measurements in a supersonic shear layer. AIAA Paper 91-0627.

FOURGUETTE, D. C., MUNGAL M. G. \& DIBBLE, R. W. 1991b Time yes evolution of the shear layer of a supersonic axisymmetric jet. AIAA J. 29, 1123-1130.

FOYSI, H. \& SARKAR, S. 2010 The compressible mixing layer: an LES study. Theor. Comput. Fluid Dyn. 24, 565-588.

FREUND, J. B., LELE, S. K. \& MOIN, P. 1997 Direct simulation of a yes supersonic round turbulent shear layer. AIAA Paper 97-0760.

FREUND, J. B., LELE, S. K. \& MOIN, P. 2000 Compressibility effects in a yes turbulent annular mixing layer. Part 1. Turbulence and growth rate. $J$. Fluid Mech. 421, 229-267.

FU, S. \& LI, Q. B. 2006 Numerical simulation of compressible mixing layers. yes Intl J. Heat Fluid Flow 27, 895-901.

FUJIWARA, H., MATSUO, Y. \& ARAKWA, C. 2000 A turbulence model for yes the pressure-strain correlation term accounting for compressibility effects. Int. J. Heat Fluid Flow 21, 354-358.

GATSKI, T. B. \& BONNET, J. P. 2009 Compressibility, turbulence and high yes speed flows, 1st ed. Elsevier Science. ISBN: 978-0-08-044565-6.

GLAWE, D. D. \& SAMIMY, M. 1993 Dispersion of solid particles in yes compressible mixing layers. J. Propul. Power 9(1), 83-89.

GÖEBEL, S. G. \& DUTTON, J. C. 1991 Experimental study of compressible yes turbulent mixing layers. AIAA J. 29, 538-546.

GÖEBEL, S. G., DUTTON. C., KRIER, H. \& RENIE, J. P. 1990 Mean and yes turbulent velocity measurements of supersonic mixing layers. Exps. Fluids 8, 263-272.

GÖRTLER, H. 1942 Berechnung von aufgaben der freien trubulenz auf no grund eines neuen näherungsansatzes. Z. angew. Math. Mech. 22, 244-254.

GRUBER, M. R., MESSERSMITH, N. L. \& DUTTON, J. C. 1992 Three- yes dimensional velocity measurements in a turbulent, compressible mixing layer. AIAA Paper 92-3544.

GRUBER, M. R., MESSERSMITH, N. L. \& DUTTON, J. C. 1993 Three- yes dimensional velocity field in a compressible mixing layer. AIAA J. 31, 2061-2067.

GUIRGUIS, R. H. 1988 Mixing enhancement in supersonic shear layers: III. yes Effect of convective Mach number. AIAA Paper 88-0701. 
GUIRGUIS, R. H., GRINSTEIN, F. F., YOUNG, T. R., ORAN, E. S., yes KAILASANATH, K. \& BORIS, J. P. 1987 Mixing enhancement in supersonic shear layers. AIAA Paper 87-0373.

HADJADJ, A., YEE, H. C. \& SJÖGREEN, B. 2011 LES of temporally yes evolving mixing layers by an eighth-order filter scheme. Int. J. Numer. Methods Fluids.

HALL, J. L., DIMOTAKIS, P. E. \& ROSEMANN, H. 1991 Experiments in yes non-reacting compressible shear layers. AIAA J. 31, $2247-2254$.

HATAUE, I. 1990 Study of compressibility effects in mixing layer by yes numerical simulation. AIAA Paper 90-1464.

HATAUE, I. \& TAKAMI, H. 1992 Study of compressibility effects in three- yes dimensional mixing layer by numerical simulation. AIAA Paper 923442.

HEDGES, L. S. \& EBERHARDT, D. S. 1993 Comparison of confined, yes compressible, spatially developing mixing layers with temporal mixing layers. AIAA J. 31(11), 1977-1983.

HERMANSON, J. C. \& WINTER, M. 1990 Laser-induced fluorescence yes imaging of supersonic shear flow. AIAA Paper 90-0501.

IKAWA, H. \& KUBOTA, T. 1975 Investigation of supersonic turbulent mixing yes layer with zero pressure gradient. AIAA J. 13, 566-572.

ISLAND, T. C., URBAN, W. D. \& MUNGAL, M. G. 1998 Mixing yes enhancement in compressible shear layers via sub-boundary layer disturbances. Phys. Fluids 10(4), 1008-1020.

JACKSON, T. L. \& GROSCH, C. E. 1990 Absolute / convective instabilities yes and the convective Mach number in a compressible mixing layer. Phys. Fluids A 2, 949.

KENNEDY, C. A. \& GATSKI, T. B. 1994 Self-similar supersonic variable- yes density shear layers in binary systems. Phys. Fluids 6, 662.

KIM, S. 1990 A new mixing length model for supersonic shear layers. AIAA Yes Paper 90-0018.

KIM, J. \& PARK, S. O. 2010 New compressible turbulence model for free No and wall-bounded shear layers. J. Turbulence 11.

KLINE, S. J., CANTWELL, B. J. \& LILLEY, G. M. 1983 The 1980-81 Yes AFOSR-HTTM-Stanford conference on complex turbulent flows: Comparison of computation and experiment - Volume I. AFOSR-TR83-1001.

KOURTA, A. \& SAUVAGE, R. 2002 Computation of supersonic mixing layers. Phys. Fluids 14 (11), 3790-3797.

KOZUSKO, F., GROSCH, C. E., JACKSON, T. L., KENNEDY, C. A. \& Yes GATSKI, T. B. 1996 The structure of variable property, compressible mixing layers in binary gas mixtures. Phys. Fluids 8(7), 1945.

KWOK, F. T., ANDREW, P. L., NG, W. F. \& SCHETZ, J. A. 1990 Yes Experimental investigation of a supersonic shear layer with slot injection of helium. AIAA Paper 90-0093.

KWOK, F. T., ANDREW, P. L., NG, W. F. \& SCHETZ, J. A. 1991 Yes Experimental investigation of a supersonic shear layer with slot injection of helium. AIAA J. 29, 1426-1435.

LEEP, L. J., DUTTON, J. C. \& BURR, R. F. 1993 Three-dimensional Yes simulations of compressible mixing layers: Visualizations and statistical analysis. AIAA J. 31(11), 2039-2046. 
LELE, S. 1989 Direct numerical simulation of compressible free shear flows. $\mid$ Yes AIAA Paper 1989-0374.

LELE, S. K. 1994 Compressibility effects on turbulence. Ann. Rev. Fluid Yes Mech. 26, 211-254.

LI, Q. \& FU, S. 2003 Numerical simulation of high-speed planar mixing Yes layer. Comp. Fluids 32, 1357-1377.

LI, D., FAN, J. \& CEN, K. 2012 Direct numerical simulation of the Yes entrainment coefficient and turbulence properties for compressible spatially evolving axisymmetric jets. Fuel 102, 470-477.

LIEPMANN, H. W. \& LAUFER, J. 1947 Investigation of free turbulent Yes mixing. NACA Tech. Note, no. 1257.

LIOU, T., LIEN, W. \& HWANG, P. 1995 Compressibility effects and mixing Yes enhancement in turbulent free shear flows. AIAA J. 33, 2332-2338.

LU, G. \& LELE, S. K. 1994 On the density ratio effect on the growth rate of Yes a compressible mixing layer. Phys. Fluids 6, 1073-1075.

LU, P. J. \& WU, K. C. 1991 Numerical investigation on the structure of a Yes confined supersonic mixing layer. Phys. Fluids A 3, 3063.

LUI, C. \& LELE, S. K. 2001 Direct numerical simulation of spatially Yes developing, compressible, turbulent mixing layers. AIAA Paper 20010291.

MAIDI, M. \& LESIEUR, M. 2005 Large eddy simulations of spatially growing No subsonic and supersonic turbulent round jets. J. Turbulence 6.

MARTHA, C. S., BLAISDELL, G. A. \& LYRINTZIS, A. S. 2011 Large eddy Yes simulations of 2-D and 3-D spatially developing mixing layers. AIAA Paper 2011-830.

MAYDEW, R. C. \& REED, J. F. 1963 Turbulent mixing of axisymmetric Yes compressible jets (in the half-jet region) with quiescent air. Sandia Corp. Report SC-4764(RR).

MESSERSMITH, N. L. \& DUTTON, J. C. 1996 Characteristic features of Yes large structures in compressible mixing layers. AIAA J. 34, 1814-1821.

MESSERSMITH, N. L., GOEBEL, S. G., RENIE, J. P., DUTTON, J. C. \& yes KRIER, H. 1990 Investigation of supersonic mixing layers. J. Propul. Power 6(4), 353-354.

MORRISETTE, E. L., BIRCH, S. F. \& WAGNER, R. D. 1973 Mean flow and yes turbulence measurements in a Mach 5 free shear layer. In Joint Meeting of Fluid Mechanics on Mixing, 79-82.

NAUGHTON, J. W., CATTAFESTA, L. N. \& SETTLES, G. S. 1993 yes Experiments on the enhancement of compressible mixing via streamwise vorticity, Part II - Vortex strength assessment and seed particle dynamics. AIAA Paper 93-0742.

NAUGHTON, J. W., CATTAFESTA, L. N. \& SETTLES, G. S. 1997 An yes experimental study of compressible turbulent mixing enhancement in swirling jets. J. Fluid Mech. 330, 271-305.

NIXON, D., KEEFE, L. R. \& KUHN, G. D. 1990 The effects of compressibility yes on a supersonic mixing layer. AIAA Paper 90-0706.

NUDING, J. R. 1996 Interaction of compressible shear layers with shock yes waves: an experimental study, Part I. AIAA Paper 96-4515.

OBERKAMPF, W. L. \& BARONE, M. F. 2004 Measures of agreement yes between computation and experiment: Validation metrics. AIAA Paper 2004-2626. 
OERTLER 1983 Coherent structures producing Mach waves inside and outside of the supersonic jet. In Structure of Complex Turbulent Shear Flow (ed. R. Dumas \& L. Fulachier) 1983. Springer-Verlag, ISBN: 0387121560.

$\mathrm{OH}$, C. K. \& LOTH, E. 1995 Unstructured grid simulations of spatially yes evolving supersonic shear layers. AIAA J. 33(7), 1229-1238.

OLSEN, M. G. \& DUTTON, J. C. 2003 Planar velocity measurements in a yes weakly compressible mixing layer. J. Fluid Mech. 486, 51-77.

OSLAND, T. C., URBAN, W. D. \& MUNGAL, M. G. 1996 Quantitative scalar yes measurements in compressible mixing layers. AIAA Paper 96-0685.

PANTANO, C. \& SARKAR, S. 2002 A study of compressibility effects in the yes high-speed turbulent shear layer using direct simulation. J. Fluid Mech. 451, 329-371.

PAPAMOSCHOU, D. 1991 Structure of the compressible turbulent shear yes layer. AIAA J. 29, 680.

PAPAMOSCHOU, D. 1992 Effect of three-dimensionality on compressible yes mixing. J. Propul. Power 8(1), 247-249.

PAPAMOSCHOU, D. 1993 Model for entropy production and pressure yes variation in confined turbulent mixing. AIAA J. 31(9), 1643-1650.

PAPAMOSCHOU, D. 1994 Thrust loss due to supersonic mixing. J. Propul. yes Power 10, 804.

PAPAMOSCHOU, D. 1995 Evidence of shocklets in a counterflow yes supersonic shear layer. Phys. Fluids 7, 233-235.

PAPAMOSCHOU, D. \& BUNYAJITRADULYA, A. 1997 Evolution of large yes eddies in compressible shear layers. Phys. Fluids A 9, 756-765.

PAPAMOSCHOU, D. \& ROSHKO, A. 1986 Observations of supersonic free yes shear layers. AIAA Paper 86-0162.

PAPAMOSCHOU, D. \& ROSHKO, A. 1988 The compressible turbulent yes shear layer: an experimental study. J. Fluid Mech. 197, 453-477.

PETRIE, H. L., SAMIMY, M. \& ADDY, A. L. 1985 A study of compressible yes turbulent free shear layers using laser Doppler velocimetry. AIAA Paper 85-0177.

PLESNIAK, M. W. \& JOHNSTON, J. P. 1988 The effects of stabilizing and no destabilizing curvature on a plane mixing layer. In Transport phenomena in Turbulent Flows: Theory, experiment, and numerical simulation (ed. M. Hirata \& N. Kaskagi). Hemisphere Publishing Corp., 377-390.

POGGIE, J. 1995 On the control of a compressible, reattaching shear layer. no PhD Thesis, Princeton University, Princeton, NJ.

POGGIE, J. \& SMITS, A. J. 1996 Large-scale coherent turbulence Yes structures in a compressible mixing layer flow. AIAA Paper 96-0436.

PUI, N. K. \& GARTSHORE I. S. 1979 Measurements of the growth rate and no structure in plane turbulent mixing layers. J. Fluid Mech. 91, 111-130.

RAGAB, S. A. \& SHEEN, S. 1990 Numerical simulation of a compressible Yes shear layer. AIAA Paper 90-1669.

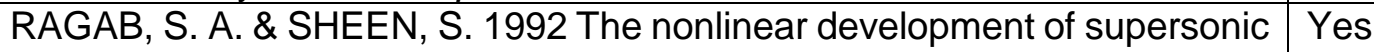
instability waves in a mixing layer. J. Fluid Mech. 486, 51-77.

RAGAB, S. A. \& WU, J. L. 1989 Linear instabilities in two-dimensional Yes compressible mixing layers. Phys. Fluids A 1, 957-966. 
RAMASWAMY, M. \& LOTH, E. 1996 Tone excitation of a supersonic $\mid$ Yes bounded shear layer. AIAA J. 34(10), 1997-2004.

REICHERT, R. S. \& BIRINGEN, S. 2006 Numerical simulation of Yes compressible plane jets. Mech. Res. Commun. 34, 249-259.

ROSSMANN, T. 2001 An experimental investigation of high compressibility Yes mixing layers. Stanford University Tech. Report, TSD-138.

ROSSMANN, T., MUNGAL, M. G. \& HANSON, R. K. 2001 Acetone PLIF Yes and Schlieren imaging of high compressibility mixing layers. AIAA Paper 2001-0290.

ROSSMANN, T., MUNGAL, M. G. \& HANSON, R. K. 2002 Character of Yes Mach wave radiation and convection velocity estimation in supersonic shear layers. AIAA Paper 2002-2571.

SABIN, C. M. 1965 An analytical and experimental study of the plane, Yes incompressible, turbulent free-shear layer with arbitrary velocity ratio and pressure gradient. Trans. ASME D 87, 421.

SAMIMY, M. \& ELLIOTT, G. S. 1990 Effects of compressibility on the yes characteristics of free shear layers. AIAA J. 28, 439-445.

SAMIMY, M. \& LELE, S. K. 1990 Particle-laden compressible free shear yes layers. AIAA Paper 90-1977.

SAMIMY, M., REEDER, M. F. \& ELLIOTT, G. S. 1992 Compressibility yes effects on large structures in free shear flows. Phys. Fluids A 4, 12511258.

SANDHAM, N. D. \& REYNOLDS, W. C. 1990 Compressible mixing layer: yes linear theory and direct simulation. AIAA J. 28, 618-624.

SANDHAM, N. D. \& REYNOLDS, W. C. 1991 Three-dimensional yes simulations of large eddies in the compressible mixing layer. J. Fluid Mech. 224, 133-158.

SARKAR, S. 2004 Modeling compressibility effects in high-speed turbulent yes flows. NAG-1-02028.

SARKAR, S. \& BALAKRISHNAN, L. 1990 Application of a Reynolds stress yes turbulence model to the compressible shear layer. NASA Contractor Report 182002. ICASE Report No. 90-18.

SARKAR, S., ERLEBACHER, G. \& HUSSAINI, M. Y. 1991 Direct simulation yes of compressible turbulence in a shear flow. Theoret. Comput. Fluid Dyn. 2, 291-305.

SCHLICHTING, H. 1979 Boundary-Layer Theory, 7th ed. McGraw - Hill, yes New York.

SETTLES, G. S. \& DODSON, L. J. 1993 Hypersonic turbulent boundary- yes layer and free shear database. NASA Contractor Report 177610.

SHAU, Y. R., DOLLING, D. S. \& CHOI, K. Y. 1993 Organized structure in a yes compressible turbulent shear layer. AIAA J. 31(8), 1398-1405.

SIMON, F., DECK, S., GUILLEN, P., SAGAUT, P. \& MERLEN, A. 2007 yes Numerical simulation of the compressible mixing layer past an axisymmetric trailing edge. J. Fluid Mech. 591, 215-253.

SIRIEIX, M. \& SOLIGNAC, J. L. 1966 Contribution a l'etude experimentale no de la couche de melange turbulent isobare d'un ecoulment supersonique. In Separated Flows, AGARD CP4.

SLESSOR, M. D. 1998 Aspects of turbulent-shear-layer dynamics and yes mixing. PhD thesis, California Institute of Technology. 
SLESSOR, M. D., ZHUANG, M. \& DIMOTAKIS, P. E. 2000 Turbulent shear- $\mid$ yes layer mixing: Growth-rate compressibility scaling. J. Fluid Mech. 414, 35-45.

SMITS, A. J. \& DUSSAUGE, J. P. 2006 Turbulent shear layers in yes supersonic flow. Springer. ISBN 0387263055.

SPARKS, C. A. \& WU, X. 2008 Nonlinear development of subsonic modes yes on compressible mixing layers: a unified strongly nonlinear criticallayer theory. J. Fluid Mech. 614, 105-144.

STRYKOWSKI, P. J., KROTHAPALLI, A. \& JENDOUBI, S. 1996 The effect yes of counterflow on the development of compressible shear layers. $J$. Fluid Mech. 308, 63-96.

STRYKOWSKI, P. J., KROTHAPALLI, A. \& WISHART, D. 1993 yes Enhancement of mixing in high-speed heated jets using a counterflowing nozzle. AIAA J. 31, 2033-2038.

TAM, C. K. W. 1971 Directional acoustic radiation from a supersonic jet yes generated by shear layer instability. J. Fluid. Mech. 46, 757-768.

TAM, C. K. W. \& HU, F. Q. 1989 The instability and acoustic wave modes yes of supersonic mixing layers inside a rectangular channel. J. Fluid Mech. 203, 51-76.

THUROW, B., LEMPERT, W. \& SAMIMY, M. $2000 \mathrm{MHz}$ rate imaging of yes large-scale structures within a high-speed axisymmetric jet. AIAA Paper 2000-0659.

THUROW, B., SAMIMY, M. \& LEMPERT, W. 2003 Compressibility effects yes on turbulence structures of axisymmetric mixing layers. Phys. Fluids $15,1755$.

THUROW, B. S., BLOHM, M., LEMPERT, W. R. \& SAMIMY, M. 2005 High yes repetition rate planar velocity measurements in a Mach 2.0 compressible axisymmetric jet. AIAA Paper 2005-0515.

THUROW, B. S., JIANG, N., KIM, J. H., LEMPERT, W. \& SAMIMY, M. 2008 yes Issues with measurements of the convective velocity of large-scale structures in the compressible shear layer of a free jet. Phys. Fluids 20, 066101.

URBAN, W. D., WATANABE, S. \& MUNGAL, M. G. 1998 Velocity field of yes the planar shear layer: Compressibility effects. AIAA Paper 98-0697.

VIEGAS, J. R. \& RUBESIN, M. W. 1991 A comparative study of several yes compressibility corrections to turbulence models applied to highspeed shear layers. AIAA Paper 91-1783.

VISWANATHAN, K. \& MORRIS, P. J. 1992 Predictions of turbulent mixing yes in axisymmetric compressible shear layers. AIAA J. 30(6), 1529-1536.

VREMAN, A. W., SANDHAM, N. D. \& LUO, K. H. 1996 Compressible mixing yes layer growth rate and turbulence characteristics. J. Fluid Mech. 320, 235-258.

WANG, Z. \& ANDREOPOULOS, Y. 2010 Density and compressibility yes effects in turbulent subsonic jets part 1: mean velocity field. Exp. Fluids 48, 327-343.

WYGNANSKI, I. \& FIEDLER, H. E. 1970 The two-dimensional mixing yes region. J. Fluid Mech. 41, 327.

YANG, W. B., ZHUANG, F. G., SHEN, Q., et al. 2009 Experimental and yes numerical study on instability structure of the supersonic mixing layer $\left(\mathrm{M}_{\mathrm{c}}=0.5\right)$. Sci. China Ser. G 52(10), 1624-1631. 
YEE, H. C., SJÖGREEN, B. \& HADJADJ, A. 2012 Comparative study of yes three high order schemes for LES of temporally evolving mixing layers. In 50th AIAA ASM Meeting, Paper ARC-E-DAA-TN4508.

YU, K. H. \& SCHADOW, K. C. 1997 Role of large coherent structures in yes turbulent compressible mixing. Exp. Therm. Fluid Sci. 14, 75-84.

ZEMAN, O. 1990 Dilatation dissipation: The concept and application in yes modeling compressible mixing layers. Phys. Fluids $A$ 2, 178-187.

ZEMAN, O. 1992 Similarity in supersonic mixing layers. AIAA J. 30(5), yes $1277-1283$.

ZHAO, Y. X., YI, S. H., TIAN, L. F., HE, L. \& CHENG, Z. Y. 2010 Density yes field measurement and approximate reconstruction of supersonic mixing layer. Chin. Sci Bull. 55, 2004-2009.

ZHOU, Q., HE, F. \& SHEN, M. Y. 2012 Direct numerical simulation of a yes spatially developing compressible plane mixing layer: flow structures and mean flow properties. J. Fluid Mech. 711, 437-468.

ZHUANG, M., DIMOTAKIS, P. E. \& KUBOTA, T. 1990 The effect of walls yes on a spatially growing supersonic shear layer. Phys. Fluids A 2, 599604. 


\section{Appendix B: Data Qualification Rubric}

The following multi-page table assesses whether each reference contributed qualifying data. Values of "1" (green) meet the metric. Values of "0" (red) fail the metric. Each reference must meet all of the criteria to qualify, so frivolous time was not spent on a reference if it failed a metric. The references are organized by year. See Section 2.2 for an explanation of each metric.

\begin{tabular}{|c|c|c|c|c|c|c|c|c|c|c|c|c|c|c|}
\hline Author(s) & Date & 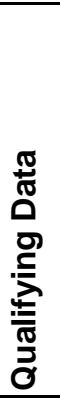 & 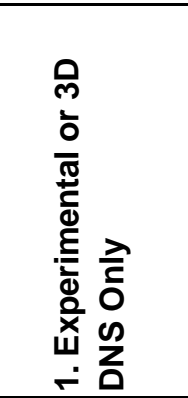 & 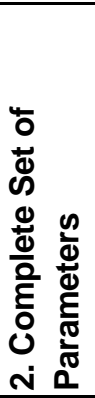 & 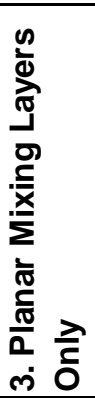 & 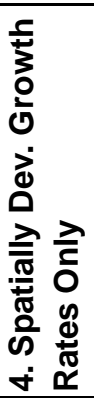 & 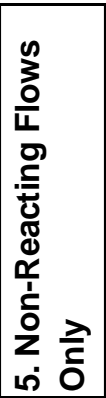 & 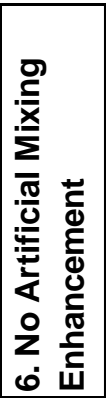 & 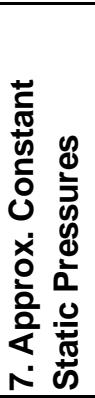 & 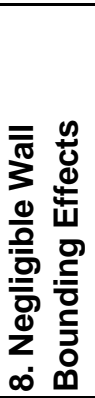 & 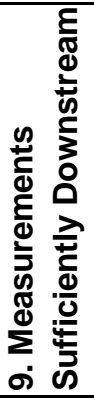 & 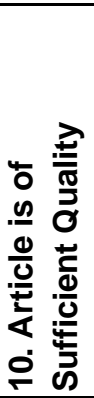 & 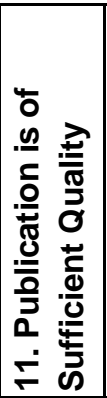 & Notes \\
\hline Liepmann \& Laufer & 1947 & 1 & Experiment & 1 & 1 & 1 & 1 & 1 & 1 & 1 & 1 & 1 & 1 & \\
\hline Maydew \& Reed & 1963 & 0 & Experiment & 1 & 0 & 1 & 1 & 1 & 0 & 1 & 1 & 1 & 1 & \\
\hline Sabin & 1965 & 0 & Experiment & 1 & 1 & 1 & 1 & 1 & 1 & 1 & 0 & 1 & 1 & $\begin{array}{l}\text { Growth rates from } \\
\text { comparison of just two x- } \\
\text { locations. }\end{array}$ \\
\hline Bradshaw & 1966 & 0 & Experiment & & 0 & & & & & & & & & \\
\hline Wygnanski \& Fiedler & 1970 & 0 & Experiment & 1 & 1 & 1 & 1 & 0 & 1 & 1 & 1 & 1 & 1 & \\
\hline Tam & 1971 & 0 & Theory & & & & & & & & & & & \\
\hline Birch \& Eggers & 1973 & 1 & Experiment & 1 & 1 & 1 & 1 & 1 & 1 & 1 & 1 & 1 & 1 & Consortium \\
\hline Morrisette \& Birch & 1973 & 0 & Experiment & & 0 & & & & & & & & & \\
\hline Brown \& Roshko & 1974 & 1 & Experiment & 1 & 1 & 1 & 1 & 1 & 1 & 1 & 1 & 1 & 1 & Seminal work \\
\hline kkawa \& Kubota & 1975 & 1 & Experiment & 1 & 1 & 1 & 1 & 1 & 1 & 1 & 1 & 1 & 1 & \\
\hline Bradshaw & 1977 & 0 & Review & & & & & & & & & & & \\
\hline Schlichting & 1979 & 0 & Textbook & & & & & & & & & & & \\
\hline Bogdanoff & 1983 & 0 & Theory & & & & & & & & & & & \\
\hline Kline et al. & 1983 & 0 & Experiment & 0 & & & & & & & & & & Consortium \\
\hline Bogdanoff & 1984 & 1 & Experiment & 1 & 1 & 1 & 1 & 1 & 1 & 1 & 1 & 1 & 1 & \\
\hline Petrie et al. & 1985 & 0 & Experiment & & & & & 0 & & & & & & Countercurrent \\
\hline
\end{tabular}




\begin{tabular}{|c|c|c|c|c|c|c|c|c|c|c|c|c|c|c|}
\hline Author(s) & Date & Qual & 1. & 2. & 3. & 4. & 5. & 6. & 7. & 8. & 9. & 10. & 11. & Notes \\
\hline Chinzei et al. & 1986 & 1 & Experiment & 1 & 1 & 1 & 1 & 1 & 1 & 1 & 1 & 1 & 1 & $\begin{array}{l}\text { Effort made during post- } \\
\text { processing transformed } \\
\text { results to constant } \\
\text { pressure. }\end{array}$ \\
\hline Dimotakis & 1986 & 0 & Theory & & & & & & & & & & & \\
\hline $\begin{array}{l}\text { Papamoschou \& } \\
\text { Roshko }\end{array}$ & 1986 & 0 & Superseded & 0 & 1 & 1 & 1 & 1 & 1 & 1 & 1 & 1 & 1 & $\begin{array}{l}\text { Results Superseded by } \\
\text { higher quality } 1988 \\
\text { paper by same authors. }\end{array}$ \\
\hline Guirguis et al. & 1987 & 0 & Numerical & & & 0 & & & & & & & & Non-DNS \\
\hline Guirguis & 1988 & 0 & Numerical & & & & & & & & & & & Non-DNS \\
\hline $\begin{array}{l}\text { Papamoschou \& } \\
\text { Roshko }\end{array}$ & 1988 & 0 & Experiment & 1 & 1 & 1 & 1 & 1 & 1 & 1 & 1 & 0 & 1 & $\begin{array}{l}\text { Significant disagreement } \\
\text { between figures for } \\
\text { certain data points }\end{array}$ \\
\hline Lele & 1989 & 0 & 3D DNS & 0 & 1 & 1 & 1 & 1 & 1 & 1 & 1 & 1 & 1 & \\
\hline Tam \& Hu & 1989 & 0 & Numerical & 0 & & & & & & & & & & Non-DNS \\
\hline Bell \& Mehta & 1990 & 0 & Experiment & 0 & & & & & & & & & & $\begin{array}{l}\text { Abnormal thickness } \\
\text { definition }\end{array}$ \\
\hline Dutton et al. & 1990 & 0 & Superseded & 1 & 1 & 1 & 1 & 1 & 1 & 1 & 1 & 0 & 1 & $\begin{array}{l}\text { Superseded by Goebel } \\
\text { \& Dutton (1991) }\end{array}$ \\
\hline Elliott \& Samimy & 1990 & 0 & Experiment & 0 & 1 & 1 & 1 & 1 & & & & 1 & 1 & \\
\hline Elliott et al. & 1990 & 0 & Experiment & 1 & 1 & 1 & 1 & 1 & 1 & 1 & 0 & 1 & 1 & $\begin{array}{l}\text { Growth rates determined } \\
\text { from comparison of just } \\
\text { two x-locations. }\end{array}$ \\
\hline Fourguette et al. & 1990 & 0 & Superseded & & & 0 & & & & & & 0 & & \begin{tabular}{|l|} 
Superseded by \\
Fourguette et al. (1991) \\
of similar title
\end{tabular} \\
\hline Goebel et al. & 1990 & 0 & Superseded & 1 & 1 & 1 & 1 & 1 & 1 & 1 & 1 & 0 & 1 & $\begin{array}{l}\text { Superseded by Goebel } \\
\text { \& Dutton (1991) }\end{array}$ \\
\hline Hataue & 1990 & 0 & 3D DNS & 0 & & & & & & & & & & \\
\hline Kim & 1990 & 0 & Theory & & & & & & & & & & & \\
\hline
\end{tabular}




\begin{tabular}{|c|c|c|c|c|c|c|c|c|c|c|c|c|c|c|}
\hline Author(s) & Date & Qual & 1. & 2. & 3. & 4. & 5. & 6. & 7. & 8. & 9. & 10. & 11. & Notes \\
\hline Kwok et al. & 1990 & 0 & Superseded & 0 & & & & & & & & 1 & 0 & $\begin{array}{l}\text { Superseded by } 1991 \\
\text { journal article of same } \\
\text { name }\end{array}$ \\
\hline Messersmith et al. & 1990 & 1 & Experiment & 1 & 1 & 1 & 1 & 1 & 1 & 1 & 1 & 1 & 1 & \\
\hline Nixon et al. & 1990 & 0 & Theory & & & & & & & & & & & \\
\hline Ragab \& Sheen & 1990 & 0 & SA \& 2D DNS & 0 & 1 & 1 & 1 & 1 & 1 & 1 & 1 & 1 & 1 & \\
\hline Samimy \& Elliot & 1990 & 1 & Experiment & 1 & 1 & 1 & 1 & 1 & 1 & 1 & 1 & 1 & 1 & \\
\hline Samimy \& Lele & 1990 & 0 & Experiment & 0 & 1 & 1 & 1 & 1 & 1 & 1 & 1 & 1 & 1 & \\
\hline $\begin{array}{l}\text { Sandham \& } \\
\text { Reynolds }\end{array}$ & 1990 & 0 & SA \& 2D DNS & & & & & & & & & 1 & 1 & \\
\hline $\begin{array}{l}\text { Sarkar \& } \\
\text { Balakrishnan }\end{array}$ & 1990 & 0 & CFD & & & & & & & & & & & \\
\hline Zeman & 1990 & 0 & Numerical & & & & & & & & & & & Non-DNS \\
\hline Zhuang et al. & 1990 & 0 & LSA & & & & & & & & & & & \\
\hline Clemens et al. & 1991 & 0 & Experiment & 0 & 1 & 1 & 1 & 1 & 1 & 1 & 1 & 1 & 1 & \\
\hline Dimotakis & 1991 & 0 & Theory & & & & & & & & & 1 & 1 & "On the convection ..." \\
\hline Dimotakis & 1991 & 0 & Review & & & & & & & & & 1 & 1 & "Turbulent free shear ..." \\
\hline Fourguette et al. & 1991 & 0 & Experiment & 1 & 1 & 0 & 1 & 1 & 1 & 1 & 1 & 1 & 1 & "Time evolution ..." \\
\hline Fourguette et al. & 1991 & 0 & Experiment & 1 & 0 & 1 & 1 & 1 & 1 & 1 & 1 & 1 & 1 & "Concentration Meas. \\
\hline Goebel \& Dutton & 1991 & 1 & Experiment & 1 & 1 & 1 & 1 & 1 & 1 & 1 & 1 & 1 & 1 & $\begin{array}{l}\text { Discard Case 1d - } \\
\text { Increased secondary } \\
\text { freestream turbulent } \\
\text { intensity }\end{array}$ \\
\hline Hall et al. & 1991 & 1 & Experiment & 1 & 1 & 1 & 1 & 1 & 1 & 1 & 1 & 1 & 1 & $\begin{array}{l}\text { Data points with } \\
\text { significantly different } \\
\text { static pressures are } \\
\text { omitted } \\
\end{array}$ \\
\hline Kwok et al. & 1991 & 0 & Experiment & & 1 & 1 & 1 & 1 & 0 & 1 & 1 & 1 & 1 & \\
\hline Lu \& Wu & 1991 & 0 & 2D DNS & 1 & 1 & 1 & 1 & 1 & 1 & 1 & 1 & 1 & 1 & \\
\hline Papamoschou & 1991 & 0 & Experiment & 0 & & & & & & & & & & \\
\hline $\begin{array}{l}\text { Sandham \& } \\
\text { Reynolds } \\
\end{array}$ & 1991 & 0 & 3D DNS & & 1 & 0 & 1 & & & & & & & \\
\hline Sarkar et al. & 1991 & 0 & 3D DNS & 0 & & & & & & & & & & \\
\hline Viegas \& Rubesin & 1991 & 0 & CFD & & & & & & & & & & & \\
\hline Abe et al. & 1992 & 0 & Experiment & 0 & & & & & & & & & & \\
\hline
\end{tabular}




\begin{tabular}{|c|c|c|c|c|c|c|c|c|c|c|c|c|c|c|}
\hline Author(s) & Date & Qual & 1. & 2. & 3. & 4. & 5. & 6. & 7. & 8. & 9. & 10. & 11. & Notes \\
\hline Barlow et al. & 1992 & 0 & Experiment & 0 & & & & & & & & & & \\
\hline Clemens \& Mungal & 1992 & 0 & Experiment & 0 & & & & & & & & & & "Effects of sidewall ..." \\
\hline Clemens \& Mungal & 1992 & 1 & Experiment & 1 & 1 & 1 & 1 & 1 & 1 & 1 & 1 & 1 & 1 & "Two- and three-dim. ..." \\
\hline Gruber et al. & 1992 & 0 & Superseded & 1 & 1 & 1 & 1 & 1 & 1 & 1 & 1 & 0 & 1 & $\begin{array}{l}\text { Superseded by Gruber } \\
\text { et al. (1993) }\end{array}$ \\
\hline Erdos et al. & 1992 & 0 & Experiment & 1 & 1 & 1 & 1 & 1 & 0 & 0 & 0 & 1 & 1 & \\
\hline Elliott et al. & 1992 & 0 & Experiment & 0 & 1 & 1 & 1 & 1 & & & & & & \\
\hline Hataue \& Takami & 1992 & 0 & 3D DNS & 0 & 1 & 1 & 1 & 1 & & & & & & \\
\hline Papamoschou & 1992 & 0 & Theory & & & & & & & & & & & \\
\hline Ragab \& Sheen & 1992 & 0 & 3D DNS & 0 & 1 & 1 & 1 & & & & & & & \\
\hline Samimy et al. & 1992 & 0 & Experiment & 0 & 1 & 1 & 1 & 1 & & & & 1 & 1 & \\
\hline $\begin{array}{l}\text { Viswanathan \& } \\
\text { Morris }\end{array}$ & 1992 & 0 & Theory & & & & & & & & & & & \\
\hline Zeman & 1992 & 0 & Theory & & & & & & & & & & & \\
\hline Bonnet et al. & 1993 & 0 & Experiment & 0 & & & & & & & & & & \\
\hline Glawe \& Samimy & 1993 & 0 & Experiment & 0 & 1 & 1 & 1 & 1 & & & & & & \\
\hline Gruber et al. & 1993 & 1 & Experiment & 1 & 1 & 1 & 1 & 1 & 1 & 1 & 1 & 1 & 1 & \\
\hline Hedges \& Eberhardt & 1993 & 0 & Numerical & 0 & & & & & & & & & & Non-DNS \\
\hline Leep et al. & 1993 & 0 & Numerical & & & 0 & & & & & & & & Non-DNS \\
\hline Naughton et al. & 1993 & 0 & Superseded & 1 & 0 & 1 & 1 & 1 & 1 & 1 & 1 & 0 & 1 & $\begin{array}{l}\text { Superseded by } \\
\text { Naughton et al. (1997) }\end{array}$ \\
\hline Papamoschou & 1993 & 0 & Numerical & & & & & & & & & & & Non-DNS \\
\hline Settles \& Dodson & 1993 & 0 & Database & & & & & & & & & 1 & 1 & \\
\hline Shau et al. & 1993 & 0 & Experiment & 1 & 1 & 1 & 1 & 1 & 0 & 1 & 1 & 1 & 1 & $\begin{array}{l}\text { Shock impingement on } \\
\text { mixing layer has } \\
\text { considerable effect }\end{array}$ \\
\hline Strykowski et al. & 1993 & 0 & Experiment & 1 & 1 & 1 & 1 & 0 & 1 & 1 & 1 & 1 & 1 & Countercurrent \\
\hline Barre & 1994 & 0 & Theory & 0 & & & & & & & & 1 & 1 & \\
\hline Barre et al. & 1994 & 0 & Experiment & 1 & 1 & 1 & 1 & 1 & 1 & 1 & 0 & 1 & 1 & \\
\hline Debisschop et al. & 1994 & 1 & Experiment & 1 & 1 & 1 & 1 & 1 & 1 & 1 & 1 & 1 & 1 & \\
\hline Kennedy \& Gatski & 1994 & 0 & Numerical & & & & & & & & & 1 & 1 & Non-DNS \\
\hline Lele & 1994 & 0 & Review & & & & & & & & & 1 & 1 & \\
\hline Lu \& Lele & 1994 & 0 & LSA & & & & & & & & & 1 & 1 & \\
\hline Papamoschou & 1994 & 0 & Theory & & & & & & & & & 1 & 1 & \\
\hline Alvi et al. & 1995 & 0 & Experiment & & & & & 0 & & & & 1 & 1 & Countercurrent \\
\hline Alvi et al. & 1995 & 0 & Experiment & & & & & 0 & & & & 1 & 1 & Countercurrent \\
\hline
\end{tabular}




\begin{tabular}{|c|c|c|c|c|c|c|c|c|c|c|c|c|c|c|}
\hline Author(s) & Date & Qual & 1. & 2. & 3. & 4. & 5. & 6. & 7. & 8. & 9. & 10. & 11. & Notes \\
\hline Bowersox \& Schetz & 1995 & 0 & Experiment & 1 & 1 & 1 & 1 & 1 & 0 & 1 & 1 & 1 & 1 & \\
\hline Buttsworth et al. & 1995 & 0 & Experiment & 1 & 1 & 1 & 1 & 1 & 1 & 1 & 1 & 0 & 1 & $\begin{array}{l}\text { Authors claim inaccurate } \\
\text { determination of edge } \\
\text { between mixing layer } \\
\text { and secondary stream } \\
\end{array}$ \\
\hline Clemens \& Paul & 1995 & 0 & Experiment & 0 & & 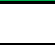 & 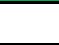 & - & - & 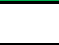 & 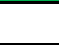 & 1 & 1 & \\
\hline Clemens \& Mungal & 1995 & 1 & Experiment & 1 & 1 & 1 & 1 & 1 & 1 & 1 & 1 & 1 & 1 & \\
\hline Elliott et al. & 1995 & 0 & Experiment & 0 & 1 & 1 & 1 & 1 & & t & & 1 & 1 & \\
\hline Liou et al. & 1995 & 0 & Numerical & & & 1 & 1 & 1 & & 1 & & 1 & 1 & Non-DNS \\
\hline Oh \& Loth & 1995 & 0 & Numerical & & & 1 & 1 & 1 & & 1 & & 1 & 1 & Non-DNS \\
\hline Papamoschou & 1995 & 0 & Experiment & & & & & 0 & & & & & - & Countercurrent \\
\hline Clemens et al. & 1996 & 0 & Experiment & 0 & 1 & 1 & 1 & 1 & & & & 1 & 1 & \\
\hline Kozusko et al. & 1996 & 0 & Numerical & & & & & & & & & & & Non-DNS \\
\hline $\begin{array}{l}\text { Messersmith \& } \\
\text { Dutton }\end{array}$ & 1996 & 0 & Experiment & 0 & 1 & 1 & 1 & 1 & & & & 1 & 1 & \\
\hline Nuding & 1996 & 0 & Experiment & & & & & & & & & 0 & & \\
\hline Osland et al. & 1996 & 1 & Experiment & 1 & 1 & 1 & 1 & 1 & 1 & 1 & 1 & 1 & 1 & \\
\hline Poggie \& Smits & 1996 & 0 & Experiment & 0 & 1 & 1 & 1 & & & & & 1 & 1 & \\
\hline Ramaswamy \& Loth & 1996 & 0 & Experiment & & & & & 0 & & & & & & \\
\hline Strykowski et al & 1996 & 0 & Experiment & & & & & 0 & & & & & & \\
\hline Vreman et al. & 1996 & 0 & 3D DNS & & & 0 & & & & & & & & \\
\hline Barre et al. & 1997 & 1 & Experiment & 1 & 1 & 1 & 1 & 1 & 1 & 1 & 1 & 1 & 1 & $\begin{array}{l}\text { Using equal static } \\
\text { pressures case only. } \\
\text { Static pressure ratios } \\
\text { presented in text used to } \\
\text { calculate density ratio. }\end{array}$ \\
\hline Brummund \& Nuding & 1997 & 0 & Experiment & & & & & 0 & & & & & & $\begin{array}{l}\text { Shock-induced mixing } \\
\text { enhancement }\end{array}$ \\
\hline Deralue \& Pope & 1997 & 0 & PDF & & & & & & & & & & & \\
\hline Freund et al & 1997 & 0 & 3D DNS & & & 0 & & & & & & & & \\
\hline Naughton et al. & 1997 & 0 & Experiment & 1 & 0 & 1 & 1 & 1 & 1 & 1 & 1 & 1 & 1 & \\
\hline $\begin{array}{l}\text { Papamoschou \& } \\
\text { Bunyajitradulya }\end{array}$ & 1997 & 0 & Experiment & 0 & & & & & & & & 1 & 1 & \\
\hline Yu \& Schadow & 1997 & 0 & Experiment & 0 & 0 & & & & & & & & & \\
\hline $\begin{array}{l}\text { AGARD Fluid } \\
\text { Dynamics Panel }\end{array}$ & 1998 & 0 & Database & & & & & & & & & & & \\
\hline
\end{tabular}




\begin{tabular}{|c|c|c|c|c|c|c|c|c|c|c|c|c|c|c|}
\hline Author(s) & Date & Qual & 1. & 2. & 3. & 4. & 5. & 6. & 7. & 8. & 9. & 10. & 11. & Notes \\
\hline Day et al. & 1998 & 0 & LSA & & & & & & & & & & & \\
\hline Island et al. & 1998 & 1 & Experiment & 1 & 1 & 1 & 1 & 1 & 1 & 1 & 1 & 1 & 1 & $\begin{array}{l}\text { Control case of mixing } \\
\text { enhancement study } \\
\text { qualifies here }\end{array}$ \\
\hline Slessor & 1998 & 0 & Experiment & 1 & 1 & 1 & 1 & 1 & 1 & 1 & 1 & 0 & 1 & $\begin{array}{l}\text { Inconsistent } \\
\text { presentation of results }\end{array}$ \\
\hline Urban et al. & 1998 & 0 & Experiment & 0 & 1 & 1 & 1 & & & & & & & \\
\hline Debiève et al. & 2000 & 0 & Experiment & 0 & & & & & & & & & & \\
\hline Doty \& McLaughlin & 2000 & 0 & Experiment & & & & & 0 & & & & & & \\
\hline Freund et al & 2000 & 0 & 3D DNS & & 0 & 0 & & & & & & & & \\
\hline Fujiwara & 2000 & 0 & CFD & & & & & & & & & & & \\
\hline Slessor et al. & 2000 & 0 & Theory & & & & & & & & & & & \\
\hline Thurow et al. & 2000 & 0 & Experiment & 0 & & & & & & & & & & \\
\hline Cottrell \& Plesniak & 2001 & 0 & RANS & & & & & & & & & & & \\
\hline Lui \& Lele & 2001 & 0 & 3D DNS & 0 & & 1 & 1 & 1 & & & & & & \\
\hline Rossmann & 2001 & 1 & Experiment & 1 & 1 & 1 & 1 & 1 & 1 & 1 & 1 & 1 & 1 & $\begin{array}{l}\text { Could not include } \\
\text { dozens of data points } \\
\text { because of insufficient } \\
\text { data. }\end{array}$ \\
\hline Rossmann et al. & 2001 & 0 & Experiment & 0 & 1 & 1 & 1 & 1 & & & & 1 & 1 & \\
\hline Kourta \& Sauvage & 2002 & 0 & 3D DNS & & & 0 & & & & & & & & \\
\hline Pantano \& Sarkar & 2002 & 0 & 3D DNS & & & 0 & & & & & & & & \\
\hline Rossmann et al. & 2002 & 0 & Experiment & 0 & & & & & & & & & & \\
\hline $\mathrm{Li} \& \mathrm{Fu}$ & 2003 & 0 & Numerical & & & & & & & & & & & Non-DNS \\
\hline Olsen \& Dutton & 2003 & 0 & Experiment & 0 & 1 & 1 & 1 & 1 & 1 & 1 & 1 & 1 & 1 & \\
\hline Thurow et al. & 2003 & 0 & Experiment & 0 & & & & & & & & & & \\
\hline Aupoix & 2004 & 0 & RANS & & & & & & & & & & & \\
\hline Sarkar & 2004 & 0 & Numerical & & & & & & & & & & & Non-DNS \\
\hline Bodi & 2005 & 0 & LES & & & & & & & & & & & \\
\hline Dimotakis & 2005 & 0 & Review & & & & & & & & & & & \\
\hline Thurow et al. & 2005 & 0 & Experiment & 0 & & & & & & & & & & \\
\hline Barone et al. & 2006 & 0 & Theory & & & & & & & & & & & \\
\hline Blohm et al. & 2006 & 0 & Experiment & 0 & & & & & & & & & & \\
\hline Fu \& Li & 2006 & 0 & Numerical & & 1 & 1 & & & & & & & & $\begin{array}{l}\text { Non-DNS (Simplified } \\
\text { gas-dynamic BGK } \\
\text { scheme) }\end{array}$ \\
\hline
\end{tabular}




\begin{tabular}{|c|c|c|c|c|c|c|c|c|c|c|c|c|c|c|}
\hline Author(s) & Date & Qual & 1. & 2. & 3. & 4. & 5. & 6. & 7. & 8. & 9. & 10. & 11. & Notes \\
\hline Oberkampf \& Barone & 2006 & 0 & Theory & & & & & & & & & & & \\
\hline Smits \& Dussuage & 2006 & 0 & Textbook & & & & & & & & & & & \\
\hline Reichert \& Biringen & 2006 & 0 & 3D DNS & 0 & 1 & 1 & 1 & 1 & & & & 1 & 1 & \\
\hline Elzawawy et al. & 2007 & 0 & Experiment & & 0 & 1 & 1 & 1 & & 1 & & 1 & 1 & \\
\hline Simon et al. & 2007 & 0 & Numerical & & & & & & & & & & & Non-DNS \\
\hline Sparks \& Wu & 2008 & 0 & Theory & & & & & & & & & & & \\
\hline Thurow et al. & 2008 & 0 & Experiment & 0 & & & & & & & & & & \\
\hline Aso et al. & 2009 & 0 & Experiment & & 0 & & & & & & & & & \\
\hline Yang et al. & 2009 & 1 & Experiment & 1 & 1 & 1 & 1 & 1 & 1 & 1 & 1 & 1 & 1 & \\
\hline Gatski \& Bonnet & 2009 & $\overline{0}$ & Textbook & & & & & & & & & & & \\
\hline Bouzada et al. & 2010 & 0 & RANS & & & & & & & & & & & \\
\hline Foysi \& Sarkar & 2010 & 0 & LES & & & & & & & & & & & \\
\hline $\begin{array}{l}\text { Wang \& } \\
\text { Andreopoulos }\end{array}$ & 2010 & 0 & Experiment & & 0 & & & & & & & & & \\
\hline Zhao et al. & 2010 & 0 & Experiment & 0 & & & & & & & & 1 & 1 & \\
\hline Hadjadj et al. & 2011 & 0 & LES & & & 0 & & & & & & & & \\
\hline Martha & 2011 & 0 & LES & & & 1 & & & & & & & & \\
\hline Yee et al. & 2011 & 0 & LES & & & 0 & & & & & & & & \\
\hline Li et al. & 2012 & 0 & 3D DNS & & 0 & & & & & & & & & \\
\hline Zhou et al. & 2012 & 1 & 3D DNS & 1 & 1 & 1 & 1 & 1 & 1 & 1 & 1 & 1 & 1 & $\begin{array}{l}\text { Periodic forcing used for } \\
\text { the computational } \\
\text { production of large } \\
\text { structures, not } \\
\text { considered artificial } \\
\text { enhancement }\end{array}$ \\
\hline
\end{tabular}




\section{Appendix C: Database}

\section{Complete, Unaltered Database}

The following multi-page table presents the complete, unaltered database. Due to its length, the table is split into two parts. The first part of the table includes top level parameters such as gas compositions, measures of compressibility, the mixing parameter, and the observed, predicted incompressible, and normalized growth rates. The second part includes details about the case definition, including primary and secondary Mach numbers, and the secondary-to-primary ratios for velocity, density, total temperature, acoustic speeds, and static pressure. A prediction for the convective velocity (as a fraction of the primary velocity) is also included. Some cases (marked by red text) assumed equal static pressures based on a comment that was made in the reference. This was done only when the static pressure ratio was required to calculate some other missing parameter. In cases where an incompressible growth rate model was not applied by the author, some other model was used in its place (typically from Papamoschou \& Roshko 1988). These cases are identified by green highlight. Since these highlighted values are all revised in Section 4.3, the model selection here was purely for preliminary plotting. As such, caution should be used when reading the normalized growth rates from this database. There were a few density-based thickness definitions for which this could not be done due to a lack of existing models. The thickness definitions are color coded by velocity-based (green), density-based (orange), and visual (blue) thicknesses. Wherever applicable, values are reported in SI units. 


\section{Part 1}

\begin{tabular}{|c|c|c|c|c|c|c|c|c|c|c|c|c|c|c|c|c|}
\hline Author & Year & Thickness Type & $M_{c}$ & $\Pi_{c}$ & $\begin{array}{c}\text { Comp. } \\
\text { Factor (Ф) }\end{array}$ & Error & $(\delta / x)_{\text {obs }}$ & $(\delta / x)_{0}$ & $\lambda r$ & $\lambda_{\mathrm{s}}$ & Gas 1 & $V_{1}$ & $\mathbf{R}_{1}$ & Gas 2 & $v_{2}$ & $\mathbf{R}_{\mathbf{2}}$ \\
\hline \multirow[t]{3}{*}{ Liepmann \& Laufer } & 1947 & Shear Layer & 0.026 & 0.033 & 0.997 & None & 0.164 & 0.165 & 1.0000 & 1.000 & Air & 1.400 & 286.9 & Air & 1.400 & 286.9 \\
\hline & & Stanford & 0.026 & 0.033 & 0.884 & None & 0.120 & 0.136 & 1.0000 & 1.000 & Air & 1.400 & 286.9 & Air & 1.400 & 286.9 \\
\hline & & Vorticity & 0.026 & 0.033 & 1.047 & None & 0.169 & 0.161 & 1.0000 & 1.000 & Air & 1.400 & 286.9 & Air & 1.400 & 286.9 \\
\hline & & & & & & & & & & & & & & & & \\
\hline \multirow[t]{2}{*}{ Birch \& Eggers (\#4 Lee) } & 1973 & Shear Layer & 0.046 & 0.057 & 1.059 & None & 0.084 & 0.079 & 0.4815 & 0.481 & Air & 1.400 & 286.9 & Air & 1.400 & 286.9 \\
\hline & & Stanford & 0.046 & 0.057 & 0.934 & None & 0.061 & 0.065 & 0.4815 & 0.481 & Air & 1.400 & 286.9 & Air & 1.400 & 286.9 \\
\hline \multirow[t]{2}{*}{ Birch \& Eggers (\#5 Hill) } & 1973 & Shear Layer & 0.882 & 1.322 & 0.578 & None & 0.082 & 0.143 & 1.0000 & 0.865 & Air & 1.400 & 286.9 & Air & 1.400 & 286.9 \\
\hline & & Stanford & 0.882 & 1.322 & 0.521 & None & 0.061 & 0.117 & 1.0000 & 0.865 & Air & 1.400 & 286.9 & Air & 1.400 & 286.9 \\
\hline \multirow[t]{17}{*}{ Brown \& Roshko } & 1974 & Visual & 0.004 & 0.011 & 1.106 & $+/-10 \%$ & 0.211 & 0.190 & 0.4472 & 0.560 & $\mathrm{He}$ & 1.667 & 2077.0 & N2 & 1.400 & 297.0 \\
\hline & & & 0.006 & 0.016 & 1.050 & $+/-10 \%$ & 0.405 & 0.385 & 0.7498 & 1.133 & $\mathrm{He}$ & 1.667 & 2077.0 & N2 & 1.400 & 297.0 \\
\hline & & & 0.009 & 0.011 & 1.141 & $+/-10 \%$ & 0.174 & 0.153 & 0.4472 & 0.449 & $\mathrm{~N} 2$ & 1.400 & 297.0 & Air & 1.400 & 286.9 \\
\hline & & & 0.012 & 0.016 & 1.108 & $+/-10 \%$ & 0.284 & 0.257 & 0.7498 & 0.755 & N2 & 1.400 & 297.0 & Air & 1.400 & 286.9 \\
\hline & & & 0.014 & 0.018 & 0.997 & $+/-10 \%$ & 0.342 & 0.343 & 1.0000 & 1.009 & N2 & 1.400 & 297.0 & Air & 1.400 & 286.9 \\
\hline & & & 0.005 & 0.011 & 0.938 & $+/-10 \%$ & 0.119 & 0.127 & 0.4472 & 0.372 & $\mathrm{~N} 2$ & 1.400 & 297.0 & $\mathrm{He}$ & 1.667 & 2077.0 \\
\hline & & & 0.007 & 0.016 & 1.179 & $+/-10 \%$ & 0.225 & 0.190 & 0.7498 & 0.560 & $\mathrm{~N} 2$ & 1.400 & 297.0 & $\mathrm{He}$ & 1.667 & 2077.0 \\
\hline & & & 0.008 & 0.018 & 1.107 & $+/-10 \%$ & 0.259 & 0.234 & 1.0000 & 0.689 & N2 & 1.400 & 297.0 & $\mathrm{He}$ & 1.667 & 2077.0 \\
\hline & & & & & & & & & & & & & & & & \\
\hline & & Vorticity & 0.004 & 0.011 & 1.127 & None & 0.107 & 0.095 & 0.4472 & 0.560 & $\mathrm{He}$ & 1.667 & 2077.0 & N2 & 1.400 & 297.0 \\
\hline & & & 0.006 & 0.016 & 0.969 & None & 0.187 & 0.193 & 0.7498 & 1.133 & $\mathrm{He}$ & 1.667 & 2077.0 & N2 & 1.400 & 297.0 \\
\hline & & & 0.007 & 0.018 & 0.433 & None & 0.134 & 0.310 & 1.0000 & 1.823 & $\mathrm{He}$ & 1.667 & 2077.0 & N2 & 1.400 & 297.0 \\
\hline & & & 0.009 & 0.011 & 1.166 & None & 0.089 & 0.076 & 0.4472 & 0.449 & $\mathrm{~N} 2$ & 1.400 & 297.0 & Air & 1.400 & 286.9 \\
\hline & & & 0.012 & 0.016 & 1.047 & None & 0.134 & 0.128 & 0.7498 & 0.755 & $\mathrm{~N} 2$ & 1.400 & 297.0 & Air & 1.400 & 286.9 \\
\hline & & & 0.014 & 0.018 & 1.052 & None & 0.180 & 0.172 & 1.0000 & 1.009 & N2 & 1.400 & 297.0 & Air & 1.400 & 286.9 \\
\hline & & & 0.005 & 0.011 & 0.956 & None & 0.060 & 0.063 & 0.4472 & 0.372 & $\mathrm{~N} 2$ & 1.400 & 297.0 & $\mathrm{He}$ & 1.667 & 2077.0 \\
\hline & & & 0.008 & 0.018 & 2.056 & None & 0.241 & 0.117 & 1.0000 & 0.689 & $\mathrm{~N} 2$ & 1.400 & 297.0 & $\mathrm{He}$ & 1.667 & 2077.0 \\
\hline
\end{tabular}




\begin{tabular}{|c|c|c|c|c|c|c|c|c|c|c|c|c|c|c|c|c|}
\hline Author & Year & Thickness Type & $M_{c}$ & $\mathbf{m}_{\mathrm{c}}$ & $\begin{array}{c}\text { Comp. } \\
\text { Factor (Ф) }\end{array}$ & Error & $(\delta / x)_{\text {obs }}$ & $(\delta / x)_{0}$ & $\lambda r$ & $\lambda_{s}$ & Gas 1 & $V_{1}$ & $\mathbf{R}_{1}$ & Gas 2 & $V_{2}$ & $\mathbf{R}_{\mathbf{2}}$ \\
\hline \multirow[t]{2}{*}{ Ikawa \& Kubota } & 1975 & Shear Layer & 0.992 & 1.562 & 0.465 & None & 0.064 & 0.138 & 1.0000 & 0.835 & Air & 1.400 & 286.9 & Air & 1.400 & 286.9 \\
\hline & & Momentum & 0.992 & 1.562 & & None & 0.007 & & 1.0000 & 0.835 & Air & 1.400 & 286.9 & Air & 1.400 & 286.9 \\
\hline \multirow[t]{3}{*}{ Bogdanoff } & 1984 & Max. Concent. & 0.137 & 0.346 & & None & 0.377 & & 1.0000 & 1.846 & $\mathrm{He}$ & 1.667 & 2077.0 & Air & 1.400 & 286.9 \\
\hline & & & 0.135 & 0.162 & & None & 0.189 & & 1.0000 & 0.906 & $\mathrm{CO} 2$ & 1.289 & 189.0 & Air & 1.400 & 286.9 \\
\hline & & & 0.101 & 0.100 & & None & 0.114 & & 1.0000 & 0.723 & SF6 & 1.094 & 56.9 & Air & 1.400 & 286.9 \\
\hline \multirow[t]{6}{*}{ Chinzei et al. } & 1986 & Stanford & 0.945 & 1.455 & 0.300 & None & 0.057 & 0.191 & 1.0000 & 0.849 & Air & 1.400 & 286.9 & Air & 1.400 & 286.9 \\
\hline & & & 0.825 & 1.283 & 0.320 & None & 0.050 & 0.156 & 0.7857 & 0.690 & Air & 1.400 & 286.9 & Air & 1.400 & 286.9 \\
\hline & & & 0.668 & 1.020 & 0.425 & None & 0.047 & 0.111 & 0.5385 & 0.494 & Air & 1.400 & 286.9 & Air & 1.400 & 286.9 \\
\hline & & & 0.589 & 0.888 & 0.479 & None & 0.043 & 0.090 & 0.4388 & 0.410 & Air & 1.400 & 286.9 & Air & 1.400 & 286.9 \\
\hline & & & 0.519 & 0.771 & 0.563 & None & 0.040 & 0.072 & 0.3605 & 0.342 & Air & 1.400 & 286.9 & Air & 1.400 & 286.9 \\
\hline & & & 0.270 & 0.378 & 0.794 & None & 0.027 & 0.034 & 0.1494 & 0.147 & Air & 1.400 & 286.9 & Air & 1.400 & 286.9 \\
\hline \multirow[t]{2}{*}{ Messersmith et al. } & 1990 & Shear Layer & 0.200 & 0.274 & 1.095 & None & 0.021 & 0.019 & 0.1173 & 0.116 & Air & 1.400 & 286.9 & Air & 1.400 & 286.9 \\
\hline & & & 0.446 & 0.641 & 0.997 & None & 0.045 & 0.045 & 0.2658 & 0.274 & Air & 1.400 & 286.9 & Air & 1.400 & 286.9 \\
\hline \multirow[t]{7}{*}{ Samimy \& Elliott } & 1990 & Shear Layer & 0.510 & 0.735 & 0.988 & None & 0.079 & 0.080 & 0.4706 & 0.447 & Air & 1.400 & 286.9 & Air & 1.400 & 286.9 \\
\hline & & & 0.640 & 0.933 & 0.767 & None & 0.077 & 0.100 & 0.6000 & 0.555 & Air & 1.400 & 286.9 & Air & 1.400 & 286.9 \\
\hline & & Vorticity & 0.510 & 0.735 & 0.979 & None & 0.093 & 0.095 & 0.4706 & 0.447 & Air & 1.400 & 286.9 & Air & 1.400 & 286.9 \\
\hline & & & 0.640 & 0.933 & 0.753 & None & 0.087 & 0.116 & 0.6000 & 0.555 & Air & 1.400 & 286.9 & Air & 1.400 & 286.9 \\
\hline & & & & & & & & & & & & & & & & \\
\hline & & Momentum & 0.510 & 0.735 & 0.960 & None & 0.016 & 0.017 & 0.4706 & 0.447 & Air & 1.400 & 286.9 & Air & 1.400 & 286.9 \\
\hline & & & 0.640 & 0.933 & 0.650 & None & 0.014 & 0.021 & 0.6000 & 0.555 & Air & 1.400 & 286.9 & Air & 1.400 & 286.9 \\
\hline \multirow[t]{6}{*}{ Goebel \& Dutton } & 1991 & Shear Layer & 0.200 & 0.273 & 0.989 & None & 0.020 & 0.020 & 0.1236 & 0.123 & Air & 1.400 & 286.9 & Air & 1.400 & 286.9 \\
\hline & & & 0.460 & 0.645 & 0.816 & None & 0.038 & 0.047 & 0.2739 & 0.282 & Air & 1.400 & 286.9 & Air & 1.400 & 286.9 \\
\hline & & & 0.690 & 1.014 & 0.564 & None & 0.059 & 0.105 & 0.6949 & 0.633 & Air & 1.400 & 286.9 & Air & 1.400 & 286.9 \\
\hline & & & 0.720 & 1.046 & 0.633 & None & 0.058 & 0.092 & 0.6000 & 0.555 & Air & 1.400 & 286.9 & Air & 1.400 & 286.9 \\
\hline & & & 0.860 & 1.241 & 0.457 & None & 0.050 & 0.109 & 0.7241 & 0.663 & Air & 1.400 & 286.9 & Air & 1.400 & 286.9 \\
\hline & & & 0.990 & 1.293 & 0.400 & None & 0.049 & 0.122 & 0.7241 & 0.742 & Air & 1.400 & 286.9 & Air & 1.400 & 286.9 \\
\hline
\end{tabular}




\begin{tabular}{|c|c|c|c|c|c|c|c|c|c|c|c|c|c|c|c|c|}
\hline Author & Year & Thickness Type & $M_{c}$ & $\Pi_{c}$ & $\begin{array}{c}\text { Comp. } \\
\text { Factor (Ф) }\end{array}$ & Error & $(\delta / x)_{\text {obs }}$ & $(\delta / x)_{0}$ & $\lambda r$ & $\lambda_{\mathrm{s}}$ & Gas 1 & $V_{1}$ & $\mathbf{R}_{1}$ & Gas 2 & $V_{2}$ & $\mathbf{R}_{\mathbf{2}}$ \\
\hline \multirow[t]{6}{*}{ Hall et al. } & 1991 & Visual & 0.962 & 2.699 & 0.234 & None & 0.100 & 0.428 & 0.8248 & 1.260 & $\mathrm{He}$ & 1.667 & 2077.0 & Argon & 1.667 & 208.0 \\
\hline & & & 0.906 & 1.882 & 0.274 & None & 0.108 & 0.394 & 0.8315 & 1.159 & $\mathrm{He}$ & 1.667 & 2077.0 & N2 & 1.400 & 297.0 \\
\hline & & & 0.511 & 0.706 & 0.570 & None & 0.114 & 0.200 & 0.6194 & 0.589 & N2 & 1.400 & 297.0 & N2 & 1.400 & 297.0 \\
\hline & & & 0.175 & 0.437 & 0.656 & None & 0.062 & 0.094 & 0.3245 & 0.278 & N2 & 1.400 & 297.0 & $\mathrm{He}$ & 1.667 & 2077.0 \\
\hline & & & 0.106 & 0.447 & 0.601 & None & 0.040 & 0.067 & 0.2225 & 0.196 & Argon & 1.667 & 208.0 & $\mathrm{He}$ & 1.667 & 2077.0 \\
\hline & & & 0.093 & 0.221 & 0.909 & None & 0.097 & 0.107 & 0.3680 & 0.314 & N2 & 1.400 & 297.0 & $\mathrm{He}$ & 1.667 & 2077.0 \\
\hline \multirow[t]{3}{*}{ Clemens \& Mungal } & 1992 & Pitot & 0.280 & 0.382 & 0.580 & $+/-10 \%$ & 0.036 & 0.062 & 0.2270 & 0.224 & Air & 1.400 & 286.9 & Air & 1.400 & 286.9 \\
\hline & & & 0.620 & 0.901 & 0.410 & $+/-10 \%$ & 0.061 & 0.149 & 0.5625 & 0.524 & Air & 1.400 & 286.9 & Air & 1.400 & 286.9 \\
\hline & & & 0.790 & 1.098 & 0.360 & $+/-10 \%$ & 0.063 & 0.175 & 0.6393 & 0.614 & Air & 1.400 & 286.9 & Argon & 1.667 & 208.0 \\
\hline Gruber et al. & 1993 & Shear Layer & 0.792 & 1.242 & 0.500 & None & 0.052 & 0.104 & 0.7094 & 0.624 & Air & 1.400 & 286.9 & Air & 1.400 & 286.9 \\
\hline \multirow[t]{5}{*}{ Debisschop et al. } & 1994 & Vorticity & 0.522 & 0.725 & 1.000 & None & 0.102 & 0.102 & 0.6327 & 0.599 & Air & 1.400 & 286.9 & Air & 1.400 & 286.9 \\
\hline & & & 0.535 & 0.753 & 0.766 & None & 0.069 & 0.091 & 0.5625 & 0.533 & Air & 1.400 & 286.9 & Air & 1.400 & 286.9 \\
\hline & & & 0.576 & 0.827 & 0.879 & None & 0.082 & 0.094 & 0.5873 & 0.550 & Air & 1.400 & 286.9 & Air & 1.400 & 286.9 \\
\hline & & & 0.638 & 0.939 & 0.798 & None & 0.072 & 0.090 & 0.5748 & 0.532 & Air & 1.400 & 286.9 & Air & 1.400 & 286.9 \\
\hline & & & 1.039 & 1.804 & 0.488 & None & 0.055 & 0.112 & 0.8018 & 0.659 & Air & 1.400 & 286.9 & Air & 1.400 & 286.9 \\
\hline \multirow[t]{5}{*}{ Clemens \& Mungal } & 1995 & Visual & 0.284 & 0.382 & 0.720 & $+/-17 \%$ & 0.055 & 0.076 & 0.2270 & 0.224 & Air & 1.400 & 286.9 & Air & 1.400 & 286.9 \\
\hline & & & 0.419 & 0.580 & 0.630 & $+/-17 \%$ & 0.090 & 0.143 & 0.4286 & 0.414 & Air & 1.400 & 286.9 & Air & 1.400 & 286.9 \\
\hline & & & 0.494 & 0.670 & 0.560 & $+/-17 \%$ & 0.100 & 0.179 & 0.5504 & 0.529 & Air & 1.400 & 286.9 & Air & 1.400 & 286.9 \\
\hline & & & 0.634 & 0.934 & 0.390 & $+/-17 \%$ & 0.073 & 0.187 & 0.5748 & 0.535 & Air & 1.400 & 286.9 & Air & 1.400 & 286.9 \\
\hline & & & 0.802 & 1.108 & 0.420 & $+/-17 \%$ & 0.090 & 0.214 & 0.6393 & 0.614 & Air & 1.400 & 286.9 & Argon & 1.667 & 208.0 \\
\hline \multirow[t]{3}{*}{ Osland et al. } & 1996 & Visual & 0.253 & 0.340 & 0.767 & None & 0.051 & 0.066 & 0.1976 & 0.195 & N2 & 1.400 & 297.0 & N2 & 1.400 & 297.0 \\
\hline & & & 0.430 & 0.320 & 0.620 & None & 0.081 & 0.131 & 0.3986 & 0.385 & N2 & 1.400 & 297.0 & N2 & 1.400 & 297.0 \\
\hline & & & 0.627 & 0.936 & 0.460 & None & 0.073 & 0.159 & 0.5038 & 0.467 & N2 & 1.400 & 297.0 & N2 & 1.400 & 297.0 \\
\hline Barre et al. & 1997 & Vorticity & 1.000 & 1.804 & 0.467 & $+/-10 \%$ & 0.052 & 0.112 & 0.8039 & 0.661 & Air & 1.400 & 286.9 & Air & 1.400 & 286.9 \\
\hline Island et al. & 1998 & Visual & 0.627 & 0.936 & 0.723 & $+/-3 \%$ & 0.115 & 0.159 & 0.5038 & 0.467 & Air & 1.400 & 286.9 & Air & 1.400 & 286.9 \\
\hline
\end{tabular}




\begin{tabular}{|c|c|c|c|c|c|c|c|c|c|c|c|c|c|c|c|c|}
\hline Author & Year & Thickness Type & $\mathbf{M}_{\mathrm{c}}$ & $\Pi_{c}$ & $\begin{array}{c}\text { Comp. } \\
\text { Factor (Ф) }\end{array}$ & Error & $(\delta / x)_{\text {obs }}$ & $(\delta / x)_{0}$ & $\lambda r$ & $\lambda_{s}$ & Gas 1 & $v_{1}$ & $\mathbf{R}_{\mathbf{1}}$ & Gas 2 & $v_{2}$ & $\mathbf{R}_{\mathbf{2}}$ \\
\hline \multirow[t]{3}{*}{ Rossmann } & 2001 & Visual & 0.930 & 1.588 & 0.399 & $+/-0.03$ & 0.090 & 0.225 & 0.8655 & 0.663 & $*$ & 1.302 & 256.8 & $\mathrm{He}$ & 1.667 & 2077.0 \\
\hline & & & 1.790 & 2.560 & 0.212 & $+/-0.04$ & 0.079 & 0.373 & 0.9763 & 1.096 & $*$ & 1.302 & 256.8 & Air & 1.400 & 286.9 \\
\hline & & & 2.640 & 4.594 & 0.228 & $+/-0.04$ & 0.075 & 0.331 & 0.9048 & 0.974 & $* *$ & 1.638 & 211.9 & $* * *$ & 1.660 & 208.6 \\
\hline Yang et al. & 2009 & Visual & 0.505 & 0.823 & 0.775 & $+/-0.5 \%$ & 0.057 & 0.074 & 0.2274 & 0.216 & Air & 1.400 & 286.9 & Air & 1.400 & 286.9 \\
\hline Zhou et al. & 2012 & Momentum & 0.700 & 0.885 & 0.350 & None & 0.0112 & 0.032 & 0.3333 & 0.333 & Air & 1.400 & 286.9 & Air & 1.400 & 286.9 \\
\hline *Gas Mixture I: & $22 \%$ & Acetone, $78 \%$ Air & & & & & & & & & & & & & & \\
\hline **Gas Mixture II: & $95 \%$ & Argon, 5\% Air & & & & & & & & & & & & & & \\
\hline *** Gas Mixture III: & $98.8 \%$ & \% Argon, $1.2 \% \mathrm{O} 2$ & & & & & & & & & & & & & & \\
\hline
\end{tabular}


Part 2

\begin{tabular}{|c|c|c|c|c|c|c|c|c|c|c|}
\hline Author & Year & Thickness Type & $M_{1}$ & $\mathbf{M}_{2}$ & $U_{2} / U_{1}$ & $\rho_{2} / \rho_{1}$ & $\mathrm{Tt}_{2} / \mathrm{Tt}_{1}$ & $a_{2} / a_{1}$ & $\mathrm{Ps}_{2} / \mathrm{Ps}_{1}$ & $U_{c} / U_{u}$ \\
\hline \multirow{3}{*}{ Liepmann \& Laufer } & 1947 & Shear Layer & 0.053 & 0.000 & 0.000 & 1.000 & 1.000 & 1.000 & 1.001 & 0.500 \\
\hline & & Stanford & 0.053 & 0.000 & 0.000 & 1.000 & 1.000 & 1.000 & 1.001 & 0.500 \\
\hline & & Vorticity & 0.053 & 0.000 & 0.000 & 1.000 & 1.000 & 1.000 & 1.001 & 0.500 \\
\hline \multirow[t]{2}{*}{ Birch \& Eggers (\#4 Lee) } & 1973 & Shear Layer & 0.140 & 0.050 & 0.350 & 1.000 & 1.000 & 1.002 & 1.003 & 0.675 \\
\hline & & Stanford & 0.140 & 0.050 & 0.350 & 1.000 & 1.000 & 1.002 & 1.003 & 0.675 \\
\hline \multirow[t]{2}{*}{ Birch \& Eggers (\#5 Hill) } & 1973 & Shear Layer & 2.090 & 0.000 & 0.000 & 0.534 & 1.000 & 1.369 & 1 & 0.578 \\
\hline & & Stanford & 2.090 & 0.000 & 0.000 & 0.534 & 1.000 & 1.369 & 1 & 0.578 \\
\hline \multirow[t]{16}{*}{ Brown \& Roshko } & 1974 & Visual & 0.010 & 0.011 & 0.382 & 7.000 & 1.000 & 0.347 & 1.001 & 0.552 \\
\hline & & & 0.010 & 0.004 & 0.143 & 7.000 & 1.000 & 0.347 & 1.001 & 0.378 \\
\hline & & & 0.029 & 0.011 & 0.382 & 1.036 & 1.000 & 0.983 & 1.001 & 0.688 \\
\hline & & & 0.029 & 0.004 & 0.143 & 1.036 & 1.000 & 0.983 & 1.001 & 0.568 \\
\hline & & & 0.029 & 0.000 & 0.000 & 1.036 & 1.000 & 0.983 & 1.001 & 0.496 \\
\hline & & & 0.029 & 0.004 & 0.382 & 0.143 & 1.000 & 2.886 & 0.999 & 0.830 \\
\hline & & & 0.029 & 0.001 & 0.143 & 0.143 & 1.000 & 2.886 & 0.999 & 0.765 \\
\hline & & & 0.029 & 0.000 & 0.000 & 0.143 & 1.000 & 2.886 & 0.999 & 0.726 \\
\hline & & & & & & 8000 & 000 & & 1001 & 0.552 \\
\hline & & Vorticity & $\begin{array}{l}0.010 \\
0.010\end{array}$ & $\begin{array}{l}0.011 \\
0.004\end{array}$ & $\begin{array}{l}0.382 \\
0.143\end{array}$ & $\begin{array}{l}1.000 \\
7.000\end{array}$ & $\begin{array}{l}1.000 \\
1.000\end{array}$ & $\begin{array}{l}0.34 / \\
0.347\end{array}$ & $\begin{array}{l}1.001 \\
1.001\end{array}$ & $\begin{array}{l}0.552 \\
0.378\end{array}$ \\
\hline & & & 0.010 & 0.000 & 0.000 & 7.000 & 1.000 & 0.347 & 1.001 & 0.274 \\
\hline & & & 0.029 & 0.011 & 0.382 & 1.036 & 1.000 & 0.983 & 1.001 & 0.688 \\
\hline & & & 0.029 & 0.004 & 0.143 & 1.036 & 1.000 & 0.983 & 1.001 & 0.568 \\
\hline & & & 0.029 & 0.000 & 0.000 & 1.036 & 1.000 & 0.983 & 1.001 & 0.496 \\
\hline & & & 0.029 & 0.004 & 0.382 & 0.143 & 1.000 & 2.886 & 0.999 & 0.830 \\
\hline & & & 0.029 & 0.000 & 0.000 & 0.143 & 1.000 & 2.886 & 0.999 & 0.726 \\
\hline \multirow[t]{2}{*}{ Ikawa \& Kubota } & 1975 & Shear Layer & 2.470 & 0.000 & 0.000 & 0.450 & 1.000 & 1.490 & 0.999 & 0.599 \\
\hline & & Momentum & 2.470 & 0.000 & 0.000 & 0.450 & 1.000 & 1.490 & 0.999 & 0.599 \\
\hline \multirow[t]{3}{*}{ Bogdanoff } & 1984 & Max. Concent. & 0.187 & 0.000 & 0.000 & 7.250 & 1.000 & 0.343 & 1.013 & 0.271 \\
\hline & & & 0.301 & 0.000 & 0.000 & 0.659 & 1.000 & 1.293 & 1.013 & 0.552 \\
\hline & & & 0.327 & 0.000 & 0.000 & 0.199 & 1.000 & 2.546 & 1.008 & 0.692 \\
\hline \multirow[t]{6}{*}{ Chinzei et al. } & 1986 & Stanford & 2.300 & 0.000 & 0.000 & 0.486 & 1.000 & 1.435 & 1 & 0.589 \\
\hline & & & 2.300 & 0.190 & 0.120 & 0.489 & 1.000 & 1.429 & 1 & 0.638 \\
\hline & & & 2.300 & 0.490 & 0.300 & 0.509 & 1.000 & 1.401 & 1 & 0.708 \\
\hline & & & 2.300 & 0.650 & 0.390 & 0.527 & 1.000 & 1.378 & 1 & 0.743 \\
\hline & & & 2.300 & 0.800 & 0.470 & 0.548 & 1.000 & 1.351 & 1 & 0.775 \\
\hline & & & 2.300 & 1.400 & 0.740 & 0.676 & 1.000 & 1.216 & 1 & 0.883 \\
\hline
\end{tabular}




\begin{tabular}{|c|c|c|c|c|c|c|c|c|c|c|}
\hline Author & Year & Thickness Type & $M_{1}$ & $M_{2}$ & $U_{2} / U_{1}$ & $\rho_{2} / \rho_{1}$ & $\mathrm{Tt}_{2} / \mathrm{Tt}_{1}$ & $a_{2} / a_{1}$ & $\mathrm{Ps}_{2} / \mathrm{Ps}_{1}$ & $U_{c} / U_{u}$ \\
\hline \multirow[t]{2}{*}{ Messersmith et al. } & 1990 & Shear Layer & 2.040 & 1.400 & 0.790 & 0.760 & 1.000 & 1.147 & 1.000 & 0.902 \\
\hline & & & 1.910 & 1.370 & 0.580 & 1.560 & 0.510 & 0.801 & 1.001 & 0.767 \\
\hline \multirow[t]{6}{*}{ Samimy \& Elliott } & 1990 & Shear Layer & 1.800 & 0.510 & 0.360 & 0.640 & 1.000 & 1.252 & 1.003 & 0.716 \\
\hline & & & 1.960 & 0.370 & 0.250 & 0.580 & 1.000 & 1.312 & 0.998 & 0.676 \\
\hline & & Vorticity & 1.800 & 0.510 & 0.360 & 0.640 & 1.000 & 1.252 & 1.003 & 0.716 \\
\hline & & & 1.960 & 0.370 & 0.250 & 0.580 & 1.000 & 1.312 & 0.998 & 0.676 \\
\hline & & Momentum & 1800 & 0.510 & 0.360 & 0.640 & 1.000 & 1252 & 1.003 & 0.716 \\
\hline & & & 1.960 & 0.370 & 0.250 & 0.580 & 1.000 & 1.312 & 0.998 & 0.676 \\
\hline \multirow[t]{6}{*}{ Goebel \& Dutton } & 1991 & Shear Layer & 2.010 & 1.380 & 0.780 & 0.760 & 1.000 & 1.144 & 0.995 & 0.898 \\
\hline & & & 1.910 & 1.360 & 0.570 & 1.550 & 0.510 & 0.803 & 0.999 & 0.762 \\
\hline & & & 1.960 & 0.270 & 0.180 & 0.570 & 1.000 & 1.320 & 0.993 & 0.647 \\
\hline & & & 2.220 & 0.430 & 0.250 & 0.580 & 0.905 & 1.316 & 1.005 & 0.676 \\
\hline & & & 2.350 & 0.300 & 0.160 & 0.600 & 0.806 & 1.290 & 0.999 & 0.633 \\
\hline & & & 2.270 & 0.380 & 0.160 & 1.140 & 0.444 & 0.937 & 1.000 & 0.566 \\
\hline \multirow[t]{6}{*}{ Hall et al. } & 1991 & Visual & 1.500 & 0.350 & 0.096 & 5.950 & 1.000 & 0.410 & 1.002 & 0.359 \\
\hline & & & 1.480 & 0.300 & 0.092 & 4.120 & 1.000 & 0.452 & 1.001 & 0.392 \\
\hline & & & 1.460 & 0.290 & 0.235 & 0.713 & 1.000 & 1.184 & 1.000 & 0.650 \\
\hline & & & 1.480 & 0.230 & 0.510 & 0.101 & 1.000 & 3.430 & 0.998 & 0.882 \\
\hline & & & 1.500 & 0.230 & 0.636 & 0.058 & 1.000 & 4.144 & 0.996 & 0.929 \\
\hline & & & 0.650 & 0.100 & 0.462 & 0.132 & 1.000 & 3.000 & 0.998 & 0.857 \\
\hline \multirow[t]{3}{*}{ Clemens \& Mungal } & 1992 & Pitot & 1.640 & 0.910 & 0.630 & 0.770 & 0.981 & 1.138 & 0.997 & 0.827 \\
\hline & & & 1.970 & 0.420 & 0.280 & 0.590 & 0.981 & 1.297 & 0.993 & 0.687 \\
\hline & & & 2.150 & 0.380 & 0.220 & 0.770 & 0.981 & 1.247 & 1.006 & 0.635 \\
\hline Gruber et al. & 1993 & Shear Layer & 2.360 & 0.270 & 0.170 & 0.460 & 1.036 & 1.469 & 0.993 & 0.665 \\
\hline \multirow[t]{5}{*}{ Debisschop et al. } & 1994 & Vorticity & 1.48 & 0.28 & 0.225 & 0.7 & 1.000 & 1.190 & 0.991 & 0.647 \\
\hline & & & 1.65 & 0.375 & 0.28 & 0.67 & 1.000 & 1.226 & 1.007 & 0.676 \\
\hline & & & 1.76 & 0.36 & 0.26 & 0.63 & 1.000 & 1.256 & 0.995 & 0.673 \\
\hline & & & 2.03 & 0.41 & 0.27 & 0.57 & 1.000 & 1.328 & 1.006 & 0.686 \\
\hline & & & 3.2 & 0.2 & 0.11 & 0.33 & 1.000 & 1.739 & 0.998 & 0.675 \\
\hline \multirow[t]{5}{*}{ Clemens \& Mungal } & 1995 & Visual & 1.64 & 0.91 & 0.63 & 0.77 & 0.981 & 1.138 & 0.997 & 0.827 \\
\hline & & & 1.52 & 0.51 & 0.4 & 0.72 & 1.004 & 1.181 & 1.004 & 0.725 \\
\hline & & & 1.5 & 0.38 & 0.29 & 0.75 & 0.956 & 1.161 & 1.010 & 0.670 \\
\hline & & & 2 & 0.4 & 0.27 & 0.59 & 0.981 & 1.308 & 1.010 & 0.683 \\
\hline & & & 2.2 & 0.39 & 0.22 & 0.77 & 0.981 & 1.259 & 1.026 & 0.635 \\
\hline \multirow[t]{3}{*}{ Osland et al. } & 1996 & Visual & 1.64 & 0.97 & 0.67 & 0.77 & 0.996 & 1.136 & 0.993 & $0.84 €$ \\
\hline & & & 1.65 & 0.98 & 0.43 & 0.71 & 1.052 & 1.168 & 0.968 & 0.739 \\
\hline & & & 2.22 & 0.54 & 0.33 & 0.53 & 0.999 & 1.369 & 0.993 & $0.71 \varepsilon$ \\
\hline
\end{tabular}




\begin{tabular}{|c|c|c|c|c|c|c|c|c|c|c|}
\hline Author & Year & Thickness Type & $M_{1}$ & $M_{2}$ & $\mathrm{U}_{2} / \mathrm{U}_{1}$ & $\rho_{2} / \rho_{1}$ & $\mathrm{Tt}_{2} / \mathrm{Tt}_{1}$ & $a_{2} / a_{1}$ & $\mathrm{Ps}_{2} / \mathrm{Ps}_{1}$ & $U_{d} / U_{1}$ \\
\hline Barre et al. & 1997 & Vorticity & 3.20 & 0.20 & 0.109 & 0.331 & 1.000 & 1.739 & 1.000 & 0.675 \\
\hline Island et al. & 1998 & Visual & 2.22 & 0.54 & 0.33 & 0.53 & 1.000 & 1.370 & 0.994 & 0.718 \\
\hline Rossmann & 2001 & Visual & 3.1 & 0.09 & 0.072 & 0.228 & 0.214 & 2.327 & 0.965 & 0.700 \\
\hline & & & 3.25 & 0.045 & 0.012 & 1.568 & 0.202 & 0.794 & 0.919 & 0.451 \\
\hline & & & 5.12 & 0.3 & 0.050 & 1.369 & 0.081 & 0.860 & 0.998 & 0.488 \\
\hline Yang et al. & 2009 & Visual & 3.51 & 1.4 & 0.6294 & 0.4023 & 1.000 & 1.578 & 1.001 & 0.856 \\
\hline Zhou et al. & 2012 & Momentum & 2.8 & 1.4 & 0.5 & 1 & 0.542 & 1 & 1.000 & 0.750 \\
\hline
\end{tabular}


Data Set \#1 (as discussed in Section 4.3.2)

\begin{tabular}{|c|c|c|c|c|c|c|c|}
\hline Author & Year & Thickness Type & $M_{c}$ & $\begin{array}{c}\text { Comp. } \\
\text { Factor (Ф) }\end{array}$ & $(\delta / x)_{\text {obs }}$ & $(\delta / x)_{0}$ & $\lambda_{\mathrm{s}}$ \\
\hline Liepmann \& Laufer & 1947 & Shear Layer & 0.026 & 0.941 & 0.164 & 0.175 & 1.000 \\
\hline Birch \& Eggers (\#4 Lee) & 1973 & Shear Layer & 0.046 & 0.999 & 0.084 & 0.084 & 0.481 \\
\hline Birch \& Eggers (\#5 Hill) & 1973 & Shear Layer & 0.882 & 0.545 & 0.082 & 0.151 & 0.865 \\
\hline Ikawa \& Kubota & 1975 & Shear Layer & 0.992 & 0.439 & 0.064 & 0.146 & 0.835 \\
\hline Messersmith et al. & 1990 & Shear Layer & 0.200 & 1.033 & 0.021 & 0.020 & 0.116 \\
\hline Messersmith et al. & 1990 & Shear Layer & 0.446 & 0.941 & 0.045 & 0.048 & 0.274 \\
\hline Samimy \& Elliott & 1990 & Shear Layer & 0.510 & 1.011 & 0.079 & 0.078 & 0.447 \\
\hline Samimy \& Elliott & 1990 & Shear Layer & 0.640 & 0.794 & 0.077 & 0.097 & 0.555 \\
\hline Goebel \& Dutton & 1991 & Shear Layer & 0.200 & 0.934 & 0.020 & 0.021 & 0.123 \\
\hline Goebel \& Dutton & 1991 & Shear Layer & 0.460 & 0.771 & 0.038 & 0.049 & 0.282 \\
\hline Goebel \& Dutton & 1991 & Shear Layer & 0.690 & 0.533 & 059 & 0.111 & 0.633 \\
\hline Goebel \& Dutton & 1991 & Shear Layer & 0.720 & & .058 & & 0.555 \\
\hline Goebel \& Dutton & 1991 & Shear Layer & 0.860 & 0.432 & 0.050 & 0.116 & 0.663 \\
\hline Goebel \& Dutton & 1991 & Shear Layer & 0.990 & 0.378 & 0.049 & 0.130 & 0.742 \\
\hline Gruber et al. & 1993 & Shear Layer & 0.792 & 0.477 & 0.052 & 0.109 & 0.624 \\
\hline Birch \& Eggers (\#4 Lee) & 973 & Stanford & 046 & & & 0.069 & 0.481 \\
\hline Birch \& Eggers (\#5 Hill) & 1973 & Stanford & 0.882 & 0.492 & .061 & 0.124 & 0.865 \\
\hline Chinzei et al. & 1986 & Stanford & 0.945 & 0.470 & 0.057 & 0.122 & 0.849 \\
\hline Chinzei et al. & 1986 & Stanford & 0.825 & 0.503 & 0.050 & 0.099 & 0.690 \\
\hline Chinzei et al. & 1986 & Stant & 668 & 63 & 047 & 0.071 & 0.494 \\
\hline Chinze & 1986 & Stan & 589 & & 043 & .059 & 0.410 \\
\hline Chinzei et al. & 1986 & Stanford & 0.519 & 0.821 & 0.040 & 0.049 & 0.342 \\
\hline Chinzei et al. & 1986 & Stanford & 0.270 & 1.284 & 0.027 & 0.021 & 0.147 \\
\hline Liepmann \& Laufer & 1947 & Vorticity & 0.026 & 0.988 & 0.169 & 0.171 & 1.000 \\
\hline Brown and Roshko & 1974 & Vorticity & .004 & 122 & 107 & 0.096 & 0.560 \\
\hline Brown and Roshko & 1974 & Vorticity & 0.006 & 0.965 & 0.187 & 0.194 & 1.133 \\
\hline Brown and Roshko & 1974 & Vorticity & 0.009 & 1.161 & 0.089 & 0.077 & 0.449 \\
\hline Brown and Roshko & 1974 & Vorticity & 0.012 & 1.042 & 0.134 & 0.129 & 0.755 \\
\hline Brown and Roshko & 1974 & Vorticity & 0.014 & 1.047 & 0.180 & 0.172 & 1.009 \\
\hline Brown and Roshko & 1974 & Vorticity & 0.005 & 0.952 & 0.060 & 0.064 & 0.372 \\
\hline Samimy \& Elliott & 1990 & Vorticity & 0.510 & 1.218 & 0.093 & 0.076 & 0.447 \\
\hline Samimy \& Elliott & 1990 & Vorticity & 0.640 & & 0.087 & 0.095 & 0.555 \\
\hline Debisschop et al. & 1994 & Vorticity & 0.522 & 0.995 & 0.102 & 0.102 & 0.599 \\
\hline Debisschop et al. & 1994 & Vorticity & 0.535 & 0.763 & 0.069 & 0.091 & 0.533 \\
\hline Debisschop et al. & 1994 & Vorticity & 0.576 & 0.875 & 0.082 & 0.094 & 0.550 \\
\hline Debisschop et al. & 1994 & Vorticity & 0.638 & 0.795 & 0.072 & 0.091 & 0.532 \\
\hline Debisschop et al. & 1994 & Vorticity & 1.039 & 0.486 & 0.055 & 0.113 & 0.659 \\
\hline Barre et al. & 1997 & Vorticity & 1.000 & 0.464 & 0.052 & 0.113 & 0.661 \\
\hline
\end{tabular}


Data Set \#2 (as discussed in Section 4.5)

\begin{tabular}{|c|c|c|c|c|c|c|c|}
\hline Author & Year & Thickness Type & $\mathbf{M}_{\mathrm{c}}$ & $\begin{array}{c}\text { Comp. } \\
\text { Factor (Ф) }\end{array}$ & $(\delta / x)_{\text {obs }}$ & $(\delta / x)_{0}$ & $\lambda_{\mathrm{s}}$ \\
\hline Liepmann \& Laufer & 1947 & Shear Layer & 0.026 & 0.941 & 0.164 & 0.175 & 1.000 \\
\hline Birch \& Eggers (\#4 Lee) & 1973 & Shear Layer & 0.046 & 0.999 & 0.084 & 0.084 & 0.481 \\
\hline Birch \& Eggers (\#5 Hill) & 1973 & Shear Layer & 0.882 & 0.545 & 0.082 & 0.151 & 0.865 \\
\hline Ikawa \& Kubota & 1975 & Shear Layer & 0.992 & 0.439 & 0.064 & 0.146 & 0.835 \\
\hline Messersmith et al. & 1990 & Shear Layer & 0.200 & 1.033 & 0.021 & 0.020 & 0.116 \\
\hline Messersmith et al. & 1990 & Shear Layer & 0.446 & 0.941 & 0.045 & 0.048 & 0.274 \\
\hline Samimy \& Elliott & 1990 & Shear Layer & 0.510 & 1.011 & 0.079 & 0.078 & 0.447 \\
\hline Samimy \& Elliott & 1990 & Shear Layer & 0.640 & 0.794 & 0.077 & 0.097 & 0.555 \\
\hline Goebel \& Dutton & 1991 & Shear Layer & 0.200 & 0.934 & 0.020 & 0.021 & 0.123 \\
\hline Goebel \& Dutton & 1991 & Shear Layer & 0.690 & 0.533 & 0.059 & 0.111 & 0.633 \\
\hline Goebel \& Dutton & 1991 & Shear Layer & 0.720 & 0.598 & 0.058 & 0.097 & 0.555 \\
\hline Goebel \& Dutton & 1991 & Shear Layer & 0.860 & 0.432 & 0.050 & 0.116 & 0.663 \\
\hline Goebel \& Dutton & 1991 & Shear Layer & 0.990 & 0.378 & 0.049 & 0.130 & 0.742 \\
\hline Gruber et al. & 1993 & Shear Layer & .792 & & & 0.109 & 0.624 \\
\hline Birch \& Eggers (\#4 Lee) & 1973 & Stanford & 0.046 & 0.881 & 0.061 & 0.069 & 0.481 \\
\hline Birch \& Eggers (\#5 Hill) & 1973 & Stanford & 0.882 & 0.492 & 0.061 & 0.124 & 0.865 \\
\hline Chinzei et al. & 1986 & Stanford & 0.945 & 0.470 & 0.057 & 0.122 & 0.849 \\
\hline Chinzei et al. & 1986 & Stanford & 0.825 & 0.503 & 0.050 & 0.099 & 0.690 \\
\hline Chinzei et al. & 1986 & Stanford & 0.668 & & 0.047 & 0.071 & 0.494 \\
\hline Chinzei et al. & 1986 & Stanford & 0.589 & 0.731 & 0.043 & 0.059 & 0.410 \\
\hline Chinzei et al. & 1986 & Stanford & 0.519 & 0.821 & 0.040 & 0.049 & 0.342 \\
\hline Liepmann \& Laufer & 1947 & Vorticity & 0.026 & 0.988 & 0.169 & 0.171 & 1.000 \\
\hline Brown and Roshko & 1974 & Vorticity & 0.004 & 1.122 & 0.107 & 0.096 & 0.560 \\
\hline Brown and Roshko & 1974 & Vorticity & 0.006 & 0.965 & 0.187 & 0.194 & 1.133 \\
\hline Brown and Roshko & 1974 & Vorticity & 0.009 & 1.161 & 0.089 & 0.077 & 0.449 \\
\hline Brown and Roshko & 1974 & Vorticity & 0.012 & 1.042 & 0.134 & 0.129 & 0.755 \\
\hline Brown and Roshko & 1974 & Vorticity & 0.014 & 1.047 & 0.180 & 0.172 & 1.009 \\
\hline Brown and Roshko & 1974 & Vorticity & 0.005 & 0.952 & 0.060 & 0.064 & 0.372 \\
\hline Samimy \& Elliott & 1990 & Vorticity & 0.640 & 0.918 & 0.087 & 0.095 & 0.555 \\
\hline Debisschop et al. & 1994 & Vorticity & 0.522 & 0.995 & 0.102 & 0.102 & 0.599 \\
\hline Debisschop et al. & 1994 & Vorticity & 0.535 & 0.763 & 0.069 & 0.091 & 0.533 \\
\hline Debisschop et al. & 1994 & Vorticity & 0.576 & 0.875 & 0.082 & 0.094 & 0.550 \\
\hline Debisschop et al. & 1994 & Vorticity & 0.638 & 0.795 & 0.072 & 0.091 & 0.532 \\
\hline Debisschop et al. & 1994 & Vorticity & 1.039 & 0.486 & 0.055 & 0.113 & 0.659 \\
\hline Barre et al. & 1997 & Vorticity & 1.000 & 0.464 & 0.052 & 0.113 & 0.661 \\
\hline
\end{tabular}




\section{Appendix D:Pertinent Article Summaries and Comments on Data Extraction}

This article offers individual reports on the content of each of the references that contributed qualifying data. Within these reports, special attention is given to the process followed to extract the data for the present database. Similar write-ups are also provided for references that presented data that did not qualify because of Qualification Metric \#10 (The paper must be of sufficient quality), except for those that were superseded by similar articles. Unless otherwise noted, "Figure" and "Table" callouts in the following summaries refer to objects in the cited document and not within the present report.

\section{Liepman \& Laufer, 1947, Investigations of free turbulent mixing}

The 1947 NACA technical note by Liepmann and Laufer is the earliest report included in this database. Within this note, the authors present both analytical and experimental investigations into the incompressible mixing layer. The analytical study provides a discussion of the fundamentals of laminar and turbulent mixing layers. Meanwhile, the experimental study established the "gold standard" at the time for the collection and presentation of mean and turbulent velocity details.

The experiment presented a single planar mixing layer formed by a jet of room temperature air at $18 \mathrm{~m} / \mathrm{s}$ mixing on one side with a stationary body of room temperature air and constrained on the other side by a solid wall. A dual hot-wire technique was used to measure all mean velocity components, all fluctuating velocity components, and all double correlations. The location of the hot-wires was known to within $\pm 0.02 \mathrm{~cm}$, and the ocular micrometer that was used to determine the distance between the hot-wires was 
accurate to $\pm 0.006 \mathrm{~cm}$. These accuracies were not carried through the calculations to determine the total uncertainty in the velocity values or the growth rates themselves.

To extract mixing layer growth rate values from this paper, Figure 13 was digitized. This figure provided the normalized velocity profile plotted against a non-dimensional location, $\eta=\sigma y / x$, with $\sigma$ defined as 12.0. During the early investigations of mixing layers, the growth rate was often reported in terms of $\sigma$, which could be determined by tuning a model until it fit the data. The value of $\sigma$ that made the model match the experiment was deemed the mixing parameter of the experiment. To match with the current database, this mixing parameter was used to back out the now-standard values of $\delta_{b}^{\prime}$, $\delta_{S}^{\prime}$, and $\delta_{\omega}^{\prime}$.

\section{Birch \& Eggers, 1973, Free turbulent shear flows volume II - Summary of data}

This reference contains the proceedings for the Langley Working Conference on Free Turbulent Shear Flows, which was held at NASA Langley Research Center on July 20-21, 1972. This conference is responsible for the so-called "Langley Curve," which was compiled from experimental data from roughly one dozen studies on free shear flows. Within Volume II, detailed velocity profile information was provided for two planar free shear flow experiments. Namely, these experiments were Test Case \#4 (Lee 1966) and Test Case \#5 (Hill \& Page 1969). These tables of velocity measurements for $x$ - and $y$ locations was transcribed into an Excel spreadsheet and processed to determine the shear layer thicknesses and Stanford thicknesses at each available x-location. A linear regression was then used to determine the growth rate of each thickness type. 


\section{Brown and Roshko, 1974, On density effects and large structures in turbulent mixing layers}

In this benchmark report, Brown and Roshko presented a thorough study of the effects of density ratio on the growth rates of turbulent mixing layers. Their apparatus was designed to allow incompressible mixing of $N_{2}$, He, and air so as to create density ratios of $1 / 7,1$, and 7 along with velocity ratios of $1 / 7,1 / \sqrt{7}$, and 0 . Measurements were taken at high pressure (7 atm), with the streams provided by 2000 psi bottles stored at room temperature which were then regulated down to the desired pressure. Shadowgraph imaging was used to determine the visual thickness of the mixing layers, while sweeps of a Pitot-static probe and a custom density probe were used to determine the vorticity thickness $\left(\delta_{\omega}\right)$ of the mixing layers. The visual thicknesses were reported to be accurate to within $10 \%$, while the combination of mean pressure and density is claimed to determine mean velocity to within $4 \%$. The authors did not propagate the uncertainty in their velocity measurements to establish overall uncertainty in their reported vorticity thickness growth rate values. The temperature of both streams was assumed to be equal at $293 \mathrm{~K}$ due to the state of the stored supply containers. Because of the incompressible nature of the experiment, uncertainty with respect to temperature is assumed negligible.

The visual thickness measurements and the vorticity thickness measurements agreed upon the conclusion that the effect of density ratio on the growth of the mixing layer is noteworthy yet less substantial than that of velocity ratio.

The mixing layer thickness growth rates were determined for the database via digitization of Figures 7, 10, 14, and 15. Slight differences emerged after the figures were digitized, however these differences were not significant enough to question the reporting 
consistency. The most direct representation of each growth rate is included in the database to minimize the effect of digitization error.

The resulting mixing layer growth rates from the experimental study displayed significant differences from prior experiments in which the density ratios were achieved from compressibility effects. This finding supported a detailed derivation of the energy equation for plane turbulent mixing layers, which suggested that "compressibility introduces effects which do not occur in the low-speed flows." Through this derivation, the authors also found that whether a density ratio arises from temperature differences or from molecular-weight differences is irrelevant.

Large coherent structures were discovered and analyzed via shadowgraph images. They were shown to grow in size as they pass downstream. The mean eddy spacing was suggested to correlate linearly with the mean thickness and not directly on velocity ratio or density ratio. The eddies terminated via amalgamation with one another, although eddy lifespan was shown to be poorly correlative.

\section{Ikawa \& Kubota, 1975, Investigation of supersonic turbulent mixing layer with zero pressure gradient}

Ikawa and Kubota studied the effects of compressibility on a turbulent mixing layer with zero pressure gradient. Their flow condition consisted of a jet at Mach 2.47 flowing over a backward-facing step such that the velocity ratio was zero. The pressure of the stagnant stream was adjusted via mass injection in the transverse direction in order to negate any static pressure gradient. Pitot pressure, static pressure, and hot-wire surveys were conducted to determine the velocity and density profiles of the mixing layer. Deviations of the static pressure were found to be within $2 \%$ of mean. According to the authors, these 
deviations "did not appreciably alter the velocity profile or the spreading rate." The density ratio was reported as 0.45 . The high-speed and low-speed flows were composed of air with equal total temperatures near room temperature. Mixing layer growth rates were reported as shear layer thicknesses as well as momentum thicknesses.

\section{Bogdanoff, 1984, Interferometric measurement of heterogeneous shear-layer spreading rates}

Bogdanoff used interferometric measurement to study the density profiles of incompressible shear layers made from various gas compositions. The apparatus was originally designed for a different purpose and therefore created some hurdles for Bogdanoff to overcome in the data reduction process. There were two side-by-side nozzles, oriented vertically downward, which exhausted incompressible streams of working gas into stagnant air. The six working gases included $\mathrm{He}, 84 \% \mathrm{He} / 16 \% \mathrm{Ar}, 62 \%$ $\mathrm{He} / 38 \% \mathrm{Ar}, \mathrm{CO}_{2}, 50 \% \mathrm{SF}_{6} / 50 \% \mathrm{~N}_{2}$, and $S F_{6}$. These working gases produced density ratios ranging from 0.199 to 7.25 . Interferometric measurements were taken at roughly four streamwise locations for each trial in order to plot the normalized density (concentration) profiles. Bogdanoff used the maximum concentration thickness $\left(\delta_{\rho m}\right)$ as a mixing layer thickness. It is calculated by joining the $20 \%$ and $80 \%$ points of the concentration profiles with a straight line and measuring the distance between the intercepts of this line with the $0 \%$ and $100 \%$ concentration levels.

Uncertainty associated with the data within the article was said to be caused by distortion of the interferogram readings as well as human error in reading the data. The distortion was said to cause errors up to \pm 0.01 for about $95 \%$ of the data and up to $\pm 0.01-0.02$ for the high-curvature regions of some profiles. For the $\mathrm{SF}_{6}$ profile, the corresponding numbers are \pm 0.02 and $\pm 0.025-0.037$. The magnitude of the human errors were not 
quantified. These uncertainties were not propagated to values corresponding to the mixing layer growth rates.

Only two of the six mixing layer growth rates were explicitly reported in the article. To determine all six growth rates, the concentration profiles had to be digitized from Figure 4 . They were then processed to find the maximum concentration thicknesses. A comparison between the digitized results and the reported results was made to confirm accuracy in the extraction process.

The primary Mach numbers were calculated from the primary velocities reported in Table 1 while assuming that the static temperature equaled the total temperature (room temperature $=293 \mathrm{~K}$ ) for these incompressible gases. Isentropic flow relations were used to determine that the error in static temperature associated with this assumption was between $0.4 \%$ and $1.4 \%$, which is well within the accuracy of the database.

Bogdanoff supported his experiment with an assessment of the effects of buoyancy and the momentum defect at the beginning of the shear layer on shear layer behavior. He concluded that his study was not significantly affected by either of these phenomena, but that some earlier experimental results (e.g. Brown \& Roshko 1974) may have been affected by buoyancy.

\section{Chinzei et al., 1986, Spreading of two-stream supersonic turbulent mixing layers}

Chinzei et al. were among the first researchers to publish data on compressibility effects in two-stream turbulent mixing layers (note the distinction from single-stream compressible turbulent mixing layers, of which many prior studies had been conducted). Both streams in their experiment were driven by a singular source of pressurized air held at room 
temperature. The high-speed stream was held at Mach 2.3 while the low-speed stream varied in speed from Mach 0 to Mach 1.4. Pressure and velocity measurements were taken from transverse Pitot and static pressure probes, and the flows were imaged using Schlieren photographs. The static pressure field was corrected to negate the effects of reflective waves to ensure that the pressure gradient was negligible, however the exact values were not reported. This correction was used as justification for Qualification Metric \#7 in the rubric.

Neither the measured mixing layer thickness growth rates nor the normalized mixing layer thickness growth rates were explicitly listed in the article. However, a plot of the Stanford mixing layer thickness versus $x$-location was provided in Figure 3. To retrieve the growth rates found in this study, the mixing layer thicknesses of Figure 3 were digitized and subjected to a linear least-squares regression. Additionally, the density ratios were not listed for any of the cases. The density ratios for these cases was derived assuming ideal gasses and equal static pressures to be

$$
\frac{\rho_{2}}{\rho_{1}}=\frac{\gamma_{2}}{\gamma_{1}}\left(\frac{a_{2}}{a_{1}}\right)^{-2}
$$

This assumption restricted the ability to confirm that the static pressure gradient was negligible, so some trust has been placed on the authors' claim.

\section{Papamoschou \& Roshko, 1988, The compressible turbulent shear layer: an experimental study}

Papamoschou and Roshko's pioneering article in 1988 marked the clearly derived the convective Mach number, $M_{c}$, which would become the chief independent variable for compressible turbulent mixing layer studies. 
Mixing layer growth rates were also normalized against estimations for the incompressible growth rate for the same velocity and density ratios in an effort to isolate the effects of compressibility. This incompressible growth rate was calculated as

$$
\delta_{p i t, 0}^{\prime}=0.14 \frac{(1-r)(1+\sqrt{s})}{1+r \sqrt{s}}
$$

where the coefficient, 0.14 , was obtained experimentally and can vary between apparatuses and thickness definitions.

Papamoschou \& Roshko also measured mixing layer growth rates for ten arrangements of various gas compositions and Mach numbers. The composition of each stream varied between $N_{2}$, Ar, and He. The total temperatures within the chambers of both streams were measured to show insignificant differences from room temperature. Additionally, the Mach number in either stream ranged between 0.2 and 3.4. These conditions allowed for velocity ratios between 0.04 and 0.93 and density ratios between 0.24 and 9.2. The ten cases tested in this study were defined in Table 1.

The mixing layer thicknesses were defined as the Pitot thickness $\left(\delta_{\text {pit }}\right)$. A traversing Pitot probe setup was used to measure the total pressure profiles of the cases at various streamwise locations. Schlieren images were taken to supplement the data. (Note: A selection of these Schlieren images was used to infer visual thickness growth rates in a 1986 article by the same authors, but these growth rates were "deemed subjective and of limited accuracy" by the authors. It appears that the authors elected to supersede their prior work with the Pitot thickness measurements of their 1988 study.)

The mixing layer Pitot thicknesses and corresponding growth rates were presented in three separate forms throughout the article. Figure 9 presented the thicknesses plotted 
against the $x$-axis and mentioned that the growth rates were calculated from linear, leastsquares regressions of data points downstream of $x=75 \mathrm{~mm}$. Figure 14 presented the calculated mixing layer growth rates plotted against $M_{c_{1}}$. Finally, Figure 16 presented the normalized mixing layer growth rates plotted against $M_{c_{1}}$. A growth rate was not presented by the authors for Case 1 because it was a wake flow and, hence, was not expected to grow linearly.

All three of the figures mentioned above were digitized in order to collect growth rate values for the database, and the process described here was performed two separate times to determine a rough order of magnitude for the accuracy associated with the digitization process. The $\mathrm{x}$-locations from Figure 9 could be read to within $2 \%$, and the thicknesses were read to within approximately $10 \%$. After digitizing each case in Figure 9, the thicknesses downstream of $x=75 \mathrm{~mm}$ were subjected to a least-squares regression to determine the growth rates. Repeating this process twice, the growth rate values determined from Figure 9 were known to within approximately $7 \%$. In comparison, the growth rate values digitized directly from Figure 14 and Figure 16 were reproducible to well within $1 \%$. The table below shows the values determined from the second of two readings. 


\begin{tabular}{c|c|c|c|c} 
& \multicolumn{3}{|c}{ Experimental Mixing Layer Thickness Growth Rates from Papamoschou \& Roshko (1988) } & \\
Case \# & $\begin{array}{c}\text { Fit Using Data for } \mathbf{x}>=\mathbf{7 5 m m} \\
\text { Digitized from Figure 9 }\end{array}$ & Digitized from Figure 14 & $\begin{array}{c}\text { Digitized from Figure 16 } \\
\text { Reverse Engineered }\end{array}$ & $\begin{array}{c}\text { \% Difference } \\
\text { Fig. 14 vs. Fig. 16 }\end{array}$ \\
\hline 1 & 0.012 & - & - & - \\
2 & 0.0341 & 0.034886 & 0.028821076 & -19.04003362 \\
3 & 0.0265 & 0.047865 & 0.028659398 & -50.19471414 \\
4 & 0.0241 & 0.027896 & 0.029051141 & 4.056887145 \\
5 & 0.0272 & 0.02593 & 0.028039935 & 7.818928606 \\
6 & 0.0217 & 0.022992 & 0.02222695 & -3.383758842 \\
7 & 0.0369 & 0.036796 & 0.038176815 & 3.683508363 \\
8 & 0.0371 & 0.038042 & 0.038746853 & 1.835821805 \\
9 & 0.0224 & 0.028102 & 0.030286342 & 7.482116872 \\
10 & 0.0692 & 0.068844 & 0.071890934 & 4.330032534
\end{tabular}

When investigating the percent differences between the growth rates reported in Figure 14 and Figure 16, some anomalies were discovered (identified with red ink in the above table). The Figure 9 data was not used in this comparison due to the relatively large digitization error, however its results show even more significant disparities. The differences between the various representations of what should be the same exact data are larger than can be explained by the experimental error reported in the article of $\pm 10 \%$ or the digitization error. Because of the irreconcilable disparities between reporting instances within the article, the otherwise suitable data points from Papamoschou \& Roshko (1988) are not included in the present database.

\section{Messersmith et al., 1990, Investigation of supersonic mixing layers}

Messersmith et al. studied reactive and nonreactive mixing layers in a dual-stream, supersonic wind tunnel. Using this apparatus, they collected data for two cases with density ratios of 0.76 and 1.56 , velocity ratios of 0.79 and 0.58 , and convective Mach numbers of 0.20 and 0.45 , respectively. The primary and secondary gases were both air, and one of the tests was run with the primary gas heated to produce a different density. Laser Doppler velocimetry (LDV) was used to measure the velocity profiles. The shear layer thickness growth rate was measured and presented in tabular format with other pertinent data. Measurement uncertainties for these growth rates were not included. 


\section{Samimy \& Elliott, 1990, Effects of compressibility on the characteristics of free shear layers}

Samimy and Elliott conducted a series of experiments at the Ohio State University Aeronautical and Astronautical Research Laboratory. This supersonic blowdown tunnel fed cold, dry, and compressed air to a pair of independently controlled streams. The top side had a nominal Mach 2 nozzle, and the bottom side had a converging nozzle. The two streams were separated by a $1^{\circ}$ splitter plate with a trailing edge thickness of $0.5 \mathrm{~mm}$. The total temperatures of the two streams were equal and fluctuated slightly around room temperature. Static pressures were measured on the top and bottom walls of the tunnel to indicate that pressure variation at the inlet was within $6 \%$ of the mean pressure for the underexpanded case (Case 2). Schlieren photographs were taken to qualitatively describe the flow. Laser Doppler Velocimetry (LDV) was used to determine the velocity profiles at a number of different streamwise locations.

The article presented two cases at convective Mach numbers of 0.51 and 0.64 . The convective Mach numbers, the Mach numbers of the individual streams, the velocity ratios, and the density ratios were provided in Table 1 . However, the density ratio for Case 2 was reported as 9.58 , which is impossible for mixing layers with equal gas composition, equal total temperature, and nearly equal static pressures. This was therefore assumed to be a typographical error and recorded in the database as 0.58 , which agrees with the other associated parameters.

Mixing layer growth rates and normalized growth rates were reported in the form of shear layer thickness, vorticity thickness, and momentum thickness. 
Within the article, momentum thicknesses and normalized momentum thicknesses were reported in Table 2. Figure 7 presented all three sets of growth rates in both graphical and text format. Figures 8 and 9 compared experiments from the literature to this experiment's normalized shear layer thickness growth rates and vorticity thickness growth rates, respectively. These two plots were digitized in order to determine the normalized growth rates used by the authors. All representations within the article agreed with one another.

The authors also measured the extent of the level and lateral turbulence fluctuations and Reynolds stresses. This article showed preliminary yet inconclusive results suggesting that these parameters are reduced when the convective Mach number is increased. A follow-up report by the same authors later that year (Elliott \& Samimy 1990) further supported this claim with the addition of a case at $M_{c}=0.86$. Unfortunately, this follow-up article did not provide mixing layer growth rate measurements, and hence, could not be added to the database.

\section{Göebel and Dutton, 1991, Experimental study of compressible turbulent mixing layers}

Goebel and Dutton's article was among the first experimental investigations to provide accurate and detailed measurements of the mean and turbulent velocity fields in developed, compressible mixing layers. In this experiment, the mixing of two air streams of equal total temperature was investigated using pressure measurements, Schlieren photographs, and a two-component laser Doppler velocimeter (LDV) system. Mixing layer thicknesses were measured according to the shear layer thickness definition. A total of seven cases were reported, with Mach numbers ranging between 0.3 and 2.35 , velocity ratios between 0.16 and 0.79 , and density ratios between 0.57 and 1.55 . All of the necessary data for these cases were provided in tabular format. 


\section{Hall et al., 1991, Experiments in non-reacting compressible shear layers}

The experiment of Hall et al. was an investigation of the validity of the convective Mach number's derivation and its use as an independent variable while also recording mixing layer growth data for dissimilar gases. The experiment employed a two-stream blowdown wind tunnel with mixtures of $N_{2}, \mathrm{He}$, and Ar gases. A majority of the cases had the high speed Mach number, $M_{1}$, near 1.5 , while some cases had $M_{1}$ near 0.6 . The low speed Mach number varied between 0.10 and 0.44 . Velocity ratios varied between 0.092 and 0.636 , while density ratios varied between 0.058 and 5.950 . The mixing layer thickness was presented as the visual thickness, which was measured from Schlieren photographs. The conditions and mixing layer growth rate measurements which were needed for the database were presented in tabular format.

The authors found that the measured convective velocities of travelling shock waves were considerably higher than those predicted by models of isentropic pressure recovery at stagnation points in the convective frame. When considering the convective Mach number, $M_{c_{1}}$, to be an averaged measure of compressibility in the flow, the authors suggest that it could still be a valid measure of the overall compressibility of the shear layer despite having different underlying physical principles.

The results showed abnormally low mixing layer growth rates for conditions characterized by very low density ratios combined with a subsonic low-speed flow interacting with a supersonic high-speed flow (Cases 7, 8, and 9). This is in contrast to the dual subsonic case with low density ratio that displayed no abnormal growth rate reduction (Case 11). 
The authors suggested that there were one or more additional elements at play that were not known.

\section{Clemens \& Mungal, 1992, Two- and three-dimensional effects in the supersonic mixing layer}

Clemens and Mungal presented planar laser Mie scattering visualizations for three cases ranging from low to moderate compressibility in an effort to analyze the two- and threedimensional nature of the turbulent structures. Additionally, they measured total pressure profiles with a Pitot probe and presented Pitot thickness growth rates in tabular format along with other pertinent data. The authors reported uncertainties of $10 \%$ in the highspeed side Pitot pressure non-uniformities, and this uncertainty was translated through to the reported normalized growth rates. The nominal values and the $10 \%$ uncertainty were presented in Figure 4.

The authors' images suggest that the large coherent structures first noted by Brown and Roshko (1974) are less prominent in cases with high compressibility. Moreover, compressibility was found to increase the degree of three-dimensional effects within the mixing layer.

Gruber et al., 1993, Three-dimensional velocity field in a compressible mixing layer Gruber et al. used a two-component laser Doppler velocimeter (LDV) to measure the streamwise, transverse, and spanwise velocity field and turbulence statistics at a number of streamwise locations in an air-to-air, two stream mixing layer of moderate compressibility. A shear layer thickness growth rate was presented in tabular format alongside the experimental conditions. This LDV study showed that the peak streamwise and spanwise turbulence intensity remains relatively constant as compressibility 
increases, while peak transverse turbulence intensity and normalized primary Reynolds shear stress both decrease with increasing compressibility. These findings imply that the primary effect of compressibility on the mixing layer is to suppress transverse velocity fluctuations.

\section{Debisschop et al., 1994, Velocity field characteristics in supersonic mixing layers}

Debisschop et al. used their 1994 article to present the results of an experimental study on compressible mixing layers. They used a blow-down, high-pressure wind tunnel to create five different supersonic-subsonic mixing layers. Air in the supersonic stream varied in Mach number from 1.48 to 3.2, while air in the subsonic stream stayed between Mach numbers of 0.2 and 0.41 . The authors assumed constant total temperatures to calculate velocity ratios between 0.11 and 0.28 and density ratios between 0.33 and 0.7 . Among these test cases, four trials had convective Mach numbers between 0.525 and 0.64 , while the fifth trial had a convective Mach number of 1.04 .

The accuracy of the instrumentation was carefully identified. The Mach numbers obtained from Pitot-static measurements had associated errors of less than $1 \%$. The total temperatures were shown to be within $5 \%$ of each other. The relative error in the measure of the interfringe was $0.3 \%$, and the resolution of the signal processor was less than $4 \mathrm{~m} / \mathrm{s}$. The resulting error for 1000 sample data was estimated to be better than $1 \%$ of the average mean velocity $(400 \mathrm{~m} / \mathrm{s})$ and $4 \%$ on the RMS quantities. These uncertainties were not propagated to the observed growth rates.

Mean velocity profiles were obtained for several positions downstream of the splitter plate by use of static and total pressure probes. The authors analyzed this data within the selfsimilar region to determine the vorticity thickness growth rate for each case. All of the 
growth rates were normalized according to the process proposed by Papamoschou and Roshko (1988) and were then reported in graphical format.

The authors also used laser-Doppler anemometry (LDA) to obtain detailed measurements of the mean and fluctuating velocity fields. The LDA measurements were shown to agree with the pressure measurements of mean velocity profiles. Furthermore, the LDA measurements confirmed that the levels of velocity fluctuation decrease with increasing convective Mach number.

\section{Buttsworth et al., 1995, A gun tunnel investigation of hypersonic free shear layers in a planar duct}

Buttsworth et al. performed an experimental study of free shear layers between a Mach 7.11 primary stream created in a gun tunnel and four Mach 3.24 secondary streams with different mixtures of $\mathrm{N}_{2}$ and $\mathrm{H}_{2}$. The shear layers were studied with a horizontal knife-edge Schlieren system and a Pitot pressure probe. The Schlieren images were used to visually study the flow characteristics, while the Pitot probe was used to evaluate mixing layer thicknesses, which were split into upper and lower portions and plotted in Figure 11. During their discussion of the data quality, the authors stated that "it is clear that the actual secondary-stream mixing layer edge is not accurately determined by the Pitot results, while the primary-stream free shear layer edge is correctly located by the Pitot pressure." For this reason (and this reason only), the cases were disqualified from the present database. 


\section{Clemens \& Mungal, 1995, Large-scale structure and entrainment in the supersonic}

mixing layer

In this highly-cited experiment, Clemens and Mungal investigated a set of planar mixing layers ranging from low $\left(M_{c}=0.28\right)$ to moderate $\left(M_{c}=0.79\right)$ compressibility using a combination of Pitot probe measurements, Schlieren photography, planar laser Mie scattering (PLMS) from a condensed alcohol fog, and planar laser-induced fluorescence (PLIF) of nitric oxide. Mixing layer growth rates were determined for the Pitot thickness and visual thickness definitions. Refer to Clemens \& Mungal (1992) for the original presentation of their Pitot thickness data. Sufficient data for the case definition and growth rate results were neatly reported in tabular format. Error bars were presented in graphical format, suggesting $\pm 10 \%$ for the normalized Pitot thickness growth rates and approximately $\pm 17 \%$ for the normalized visual thickness growth rates. The PLMS and PLIF techniques were used to study the large-scale structure, entrainment motions, and mixture fraction fluctuations of the five cases.

\section{Osland et al., 1996, Quantitative scalar measurements in compressible mixing layers}

Osland et al. studied mixing efficiency and mixing layer growth rates of planar mixing layers with low to moderate compressibility. They measured the growth rates of visual mixing layers using nitric oxide seeded planar laser-induced fluorescence (PLIF), and the mixing efficiencies were calculated from concentration measurements. Three cases were tested, with convective Mach numbers of $0.25,0.39$, and 0.63 .

Data for the three cases were provided in tabular format. The total temperatures of each flow were calculated from the provided Mach numbers and static temperatures using isentropic flow relations. Estimates of incompressible growth rates were not provided. 


\section{Barre et al., 1997, Influence of inlet pressure conditions on supersonic turbulent mixing layers}

Barre et al. investigated the effects of non-isobaric conditions within compressible turbulent mixing layers to provide a more realistic understanding of practical applications such as scramjets and near-body flows. The examined configuration mixed the high speed flow at $M_{1}=3.2$ with a low speed flow at $M_{2}=0.2$ while using head losses in the subsonic stream to adjust the pressures. Both streams were generated from a common settling chamber. Pitot-static tubes, wall pressure measurements, Schlieren imaging, and laser Doppler velocimeter (LDV) measurements were used to examine the flows. The vorticity thickness definition was used to describe the mixing layer thickness growth rates.

The measured mixing layer growth rates of the isobaric case and the non-isobaric case were both within the measurement uncertainty of $\pm 10 \%$, suggesting that the effects of the pressure conditions are not significant. The authors claimed that the only apparent effect of the initial pressure ratio is to accelerate the transition between the initial boundary-layer state and the asymptotic mixing-layer configuration. Further studies of this kind are recommended to confirm their claim.

Although the overall conclusion of the study was clearly stated, neither the case characteristics nor the measured mixing layer growth rates were well defined. The gas content of the two streams was not mentioned in the text, however it was stated that the streams had a shared origin. Therefore, it was determined that the ratios of specific gas

constants $\left(R_{2} / R_{1}\right)$, ratios of specific heats $\left(\gamma_{2} / \gamma_{1}\right)$, and total temperatures $\left(T_{t, 2} / T_{t, 1}\right)$ were all unity. 
In order to continue determining the remaining case characteristics, it was necessary to assume that the gas used was air. Using this assumption, the ratio of acoustic speeds was calculated as

$$
\frac{a_{2}}{a_{1}}=\sqrt{\left(\frac{\gamma_{2}}{\gamma_{1}}\right)\left(\frac{R_{2}}{R_{1}}\right)\left(\frac{T_{t, 2}}{T_{t, 1}}\right) \frac{\left(1+\frac{\gamma_{1}-1}{2} M_{1}^{2}\right)}{\left(1+\frac{\gamma_{2}-1}{2} M_{2}^{2}\right)}}
$$

Neither the velocity ratios nor the density ratios were listed for any of the cases. An equation for velocity ratio was determined using the definition of Mach number to be

$$
\frac{U_{2}}{U_{1}}=\frac{M_{2}}{M_{1}}\left(\frac{a_{2}}{a_{1}}\right)
$$

Similarly, an equation for density ratio was determined using the ideal gas law for two flows of the same gas to be

$$
\frac{\rho_{2}}{\rho_{1}}=\left(\frac{P_{1}}{P_{2}}\right)\left(\frac{a_{1}}{a_{2}}\right)
$$

For isobaric experiments, the ratio of static pressures is unity. The article reports that the pressure in the supersonic stream was $25 \%$ lower than the pressure in the subsonic flow for the non-isobaric case, thus $\left(P_{2} / P_{1}\right)=0.75$.

The normalized mixing layer growth rates were digitized from Figure 4. Although the exact parameters used for the incompressible growth rate estimations were not explicitly mentioned, the text did refer to an article by Papamoschou and Roshko (1988). This reference suggests that for vorticity thicknesses, the incompressible mixing layer growth rate can be calculated as

$$
\delta^{\prime}{ }_{\omega, 0}=0.085 \frac{(1-r)(1+\sqrt{s})}{1+r \sqrt{s}}
$$


where the coefficient stems from the recommendation by Brown and Roshko (1974) that the vorticity thickness is about half of the visual thickness in incompressible mixing layers. The reported normalized mixing layer growth rates and the predicted incompressible mixing layer growth rates were used together to determine the experimentally measured mixing layer growth rates.

\section{Island et al., 1998, Mixing enhancement in compressible shear layers via sub- boundary layer disturbances}

In this paper, Island et al. discuss the impressive results from their experiment on mixing enhancement. By applying a variety of small disturbance geometries within the boundary layer of the supersonic stream of a moderately compressible $\left(M_{c}=0.63\right)$ turbulent mixing layer, Island et al. were able to increase mixing layer growth rate and mixing efficiency by $47 \%$ and $7 \%$, respectively, while incurring minimal momentum losses. These disturbances were as thin as $5 \%$ of the boundary layer displacement thickness.

Planar laser Mie scattering (PLMS) was used to measure visual thicknesses of the mixing layers, wherein the thickness was defined as the distance between $5 \%$ and $95 \%$ intensity levels. This method was shown to be repeatable to within $\pm 3 \%$. Additionally, Toepler Schlieren imaging was performed to obtain clear images of the shock and turbulent structures of the mixing layers, and planar laser induced fluorescence (PLIF) was used as an additional means of imaging.

Only the unperturbed control case (Case 0) is included in the present database in order to comply with Qualification Metric \#6. The growth rate of this case was acquired by digitizing Figure 24 and fitting a linear trendline to the plot of mixing layer thickness versus streamwise location. 


\section{Slessor, 1998, Aspects of turbulent-shear-layer dynamics and mixing}

Slessor's doctorate thesis is most known for the introduction of a new compressibility parameter (see Section 3.1, page 34). In addition to this, he performed two separate experiments. The first was on the effects of inflow conditions on the mixing layer, which had interesting results that did not apply to the present database. The second experiment was a study of the effects of compressibility on mixing layer growth rates, with an interest in studying the differences between bi-supersonic, bi-subsonic, and supersonic/subsonic mixing layers.

Visual mixing layer thickness growth rates were determined from Schlieren imaging and reported in Table 4.2, however a number of discrepancies could be found that considerably diminish the credibility of the report. First, attempting to reproduce Slessor's values for $\delta_{v i s} / \delta_{0}$ by dividing the $\delta_{v i s} / x$ column by the $\delta_{0} / x$ column fails. It appears that switching the numerator and denominator (i.e. $\delta_{0} / \delta_{v i s}$ ) sufficiently resolves this issue. Second, attempting to reproduce the reported values for $\delta_{0} / x$ by using Equation 1.2 and the value mentioned in Appendix $\mathrm{B}$ for $C_{\delta}=0.174$ should be possible, however the results do not match. It appears that the reader must first double the value of the constant to $C_{\delta}=$ 0.348. Although the discrepancies found within this one table could supposedly be remedied through relatively simple means, their existence seeds doubt into the quality of the other aspects of the experiment and report. Therefore, these cases were disqualified from the present database. 


\section{Rossmann, 2001, An experimental investigation of high compressibility mixing layers}

Rossmann's doctorate thesis studied high compressibility mixing layers with a shock tube facility. By combining a variety of gases at considerably different speeds, Rossmann was able to achieve a wealth of data at compressible Mach numbers reaching as high as 2.89 . Images of the shear layer were achieved by Schlieren imaging and planar laser induced fluorescence (PLIF) of seeded tracer species. These images were used to determine the visual mixing layer growth rate.

The report presented images of dozens of cases, with convective Mach number and associated normalized growth rates presented in tabular format in Appendix D (page 202). These cases provide perhaps the strongest experimental argument for the asymptotic behavior of turbulent mixing layers at high compressibility. Unfortunately, most of them were not presented with adequate information to make complete entries into the present database.

The three cases that were studied with PLIF imaging were accompanied by enough information in tabular format to include in the database. In these cases, the case parameters were collected from Tables 5.3 and 6.2, while the normalized growth rates attained from Schlieren imaging were collected from Appendix D. This conglomeration of information relies on the assumption that the cases studied with PLIF directly correspond to the nearest cases studied with Schlieren imaging.

The uncertainty for the convective Mach number was reported as \pm 0.05 for $M_{c}<1.5$ and \pm 0.07 for $M_{c}>1.5$. The uncertainty for the normalized growth rates through Schlieren imaging was reported as \pm 0.03 for $M_{c}<1.5$ and \pm 0.04 for $M_{c}>1.5$. 
Yang et al., 2009, Experimental and numerical study on instability structure of the supersonic mixing layer $(\mathrm{Mc}=\mathbf{0 . 5})$

Yang et al. completed a study of planar mixing layers via parallel experimental and numerical efforts. The experimental effort utilized a high spatiotemporal resolution flow visualization technique called Nano-based Planar Laser Scattering (NPLS), and the numerical effort utilized two-dimensional direct numerical simulation (DNS) to reproduce the same case. The single case that they studied was a dual-supersonic mixing layer with a convective Mach number of 0.5 . The experimental measurements reported a visual mixing layer thickness growth rate averaged over 20 trials. Based on pixel size, the relative error for the growth rates was determined to be roughly $0.5 \%$.

Unfortunately, the DNS study only reported mean vortex spacing and not mixing layer growth rate. In addition to the non-perturbed case which was included in the present database, Yang et al. also studied the effect of harmonic disturbances on the mean vortex spacing of the mixing layer with equivalent aerodynamic properties.

Zhou et al., 2012, Direct numerical simulation of a spatially developing compressible plane mixing layer: flow structures and mean flow properties

Zhou et al. investigated a moderately compressible $\left(M_{c}=0.7\right)$ mixing layer using 3D DNS. The primary focus of their study was to use the numerical technique to develop threedimensional images of the turbulent structures within the mixing layer. Through this process, a progression of $\Lambda$-vortices, hairpin vortices, and "flower" structures was discovered. Along with this result, the spatial growth rate of the mixing layer's momentum thickness was determined and presented graphically. 
A considerable portion of the parameters that define the case were "hidden" within the text. The primary and secondary Mach numbers were reported as 2.8 and 1.4, respectively. The authors failed to mention the gasses that were being modeled, however they did report that the specific heat ratios of the two streams equaled 1.4, so the gasses were inferred to be air for this database. Furthermore, the authors claimed that their assumptions of "equal specific heats and temperatures" led to the simplified convective Mach number equation of $M_{c}=\left(M_{1}-M_{2}\right) / 2$, which implies that the acoustic speeds were also equivalent. Based on this, it was understood that the specific gas constants were equivalent and that the authors' equal temperatures assumption referred to static temperature. Using this, the total temperature ratio was calculated as

$$
\frac{T_{t, 2}}{T_{t, 1}}=\frac{\left(\frac{a_{2}}{a_{1}}\right)^{2}}{\left(\frac{\gamma_{2}}{\gamma_{1}}\right)\left(\frac{R_{2}}{R_{1}}\right)}\left(\frac{1+\frac{\gamma_{2}-1}{2} M_{2}^{2}}{1+\frac{\gamma_{1}-1}{2} M_{1}^{2}}\right)
$$

Since the acoustic speeds were equivalent, the velocity ratio was calculated as the ratio of Mach numbers $\left(M_{2} / M_{1}\right)$. The authors also claimed that "the pressures of both free streams are the same." Using this and the equivalent acoustic speed assumption, the density ratio was known to be unity. The remaining case definition parameters were determined using typical processes.

One point of concern for the appropriateness of this study within the database is regarding the investigators' use of periodic forcing of the mixing layer. The authors claimed that the mixing layer was "periodically forced by a pair of the linearly most unstable oblique waves of equal amplitudes." This forcing was deemed allowable because the authors cited Sandham \& Reynolds (1991), who "have shown that simulations with forcing of linear instability waves produced the development of large-scale structures similar to a fully nonlinear computation with a random initial condition." Therefore, it was understood that 
the authors used the forcing to facilitate the computational creation of large-scale structures rather than to artificially enhance the mixing growth rate. 